Supporting Information for

\title{
Antibody Mimics as Bioorthogonal Catalysts for Highly Selective Bacterial Recognition and Antimicrobial Therapy
}

Jingsheng Niu, ${ }^{\dagger \neq}$ Liangpeng Wang, ${ }^{\star,}$ Tingting Cui, ${ }^{\dagger,}$ Zhao Wang, ${ }^{\dagger,}$ Chuanqi Zhao,,$^{\dagger,}$ Jinsong Ren, ${ }^{\dagger, \ddagger}$ and Xiaogang $Q u^{*, \dagger, \ddagger}$

${ }^{\dagger}$ Laboratory of Chemical Biology and State Key Laboratory of Rare Earth Resource Utilization, Changchun Institute of Applied Chemistry, Chinese Academy of Sciences, Changchun, Jilin 130022, P. R. China

${ }^{\star}$ School of Applied Chemistry and Engineering, University of Science and Technology of China, Hefei, Anhui 230026, P. R. China

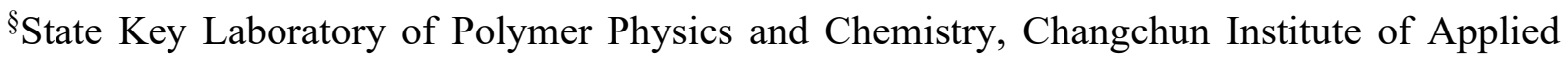
Chemistry, Chinese Academy of Sciences, Changchun, Jilin 130022, P. R. China. 


\section{Materials:}

Copper chloride dehydrate $\left(\mathrm{CuCl}_{2} \cdot 2 \mathrm{H}_{2} \mathrm{O}\right)$, tannic acid (TA), Sodium borohydride $\left(\mathrm{NaBH}_{4}\right)$, 3-morpholinopropanesulfonic acid (MOPS), (3-aminopropyl) triethoxysilane (APTES) and Tetraethoxysilane (TEOS), $N, N$-Dimethylformamide (DMF), Dimethyl sulfoxide (DMSO), 3-Nitrobenzaldehyde, I2, HBTU, HOBt, 4-alkynylbenzoic acid, 4-azidobenzoic acid, were purchased from Aladdin Chemistry Co., Ltd. (China). Ethylenediamine, $\mathrm{Boc}_{2} \mathrm{O}, 10 \% \mathrm{Pd} / \mathrm{C}$, Triethylamine, Trifluoroacetate (TFA), were purchased from Sigma-Aldrich. Tert-butanol, $\mathrm{Na}_{2} \mathrm{CO}_{3}, \mathrm{Na}_{2} \mathrm{SO}_{3}$, Ethyl acetate, $\mathrm{Na}_{2} \mathrm{SO}_{4}, \mathrm{CH}_{2} \mathrm{Cl}_{2}, \mathrm{NaHCO}_{3} . \mathrm{Na}_{2} \mathrm{SO}_{4}, \mathrm{DMF}, \mathrm{NaOH}$, were purchased from Beijing Chemicals (Beijing, China). Ultrapure water $\left(18.2 \mathrm{M} \Omega \cdot \mathrm{cm}^{-1}\right.$, Millpore Co., USA) was used throughout the experiment.

\section{Measurements:}

SEM images were obtained with a Hitachi S-4800 FE-SEM at a working voltage $10 \mathrm{kV}$ and working current $10 \mu \mathrm{A}$. TEM measurements were carried out on a TECNAI G2 equipped with EDS at $200 \mathrm{kV}$. The UV-Vis absorption spectra were measured using a JASCO V550 UV/Visible spectrophotometer (JASCO International Co., LTD., Tokyo, Japan). Fluorescence spectra were recorded on a JASCO FP-6500 spectrofluorometer. All the spectra were recorded in a $1.0 \mathrm{~cm}$ path length cell. The Zeta potentials of all samples were measured on a Zetasizer 3000HS analyzer (Malvern Instruments).

\section{Experimental Section}

Antibacterial Experiments: The Growth-inhibition assays were determined by using optical density at $600 \mathrm{~nm}$ (OD 600). After 3 hours, absorbance at $600 \mathrm{~nm}$ was recorded, the bacteria without precursor molecules and catalysts were used as control, and the culture medium 
without bacteria was considered as background. The condition of bacteria was also studied with solid medium culture. For solid medium culture, after incubation with precursor molecules and catalysts at $37^{\circ} \mathrm{C}$, the solution was diluted $10^{5}$ times with growth medium. $100 \mu \mathrm{L}$ of the diluted bacterial solutions were spread on the solid medium. The plates were cultured at $37{ }^{\circ} \mathrm{C}$ for $12 \mathrm{~h}$, and the number of colony forming units (CFUs) was counted.

Epidermal wound in vivo experiment: In order to evaluate the potential of the antibacterial system to treat wound infections, an injury model was established on the back of mice. The back of the mouse was incised (6-8 weeks), and $1 \times 10^{6}$ E. coli or $S$. aureus were injected respectively to establish the infected wound models. The mice were divided into 4 groups (three mice in each group) for each bacterium. Mice in different groups were treated with PBS buffer (Control), $\mathbf{E}-\mathbf{A b} / \mathbf{S}-\mathbf{A b}, \mathbf{4}+\mathbf{5}, \mathbf{E}-\mathbf{A b} / \mathbf{S}-\mathbf{A b}+\mathbf{4}+\mathbf{5}$, respectively. Observed the wound, took pictures, injected antibacterial materials into the wound every other day and replaced the sterile dressings. After 5 days of treatment, the mice were sacrificed and wound tissues were collected. The wound tissue was placed in $1 \mathrm{~mL}$ of sterile saline. The obtained solution was incubated overnight at $37^{\circ} \mathrm{C}$, and the number of bacteria in it was determined by plate counting. Animal research was conducted in accordance with the guidelines of the Institutional Animal Care and Use Committee.

Histology: In terms of histology, the mice were sacrificed, and wound tissues were collected after 5 days of treatment. The wound tissues treated with different nanoparticles were fixed in neutral formalin buffer, conventionally treated as paraffin, cut into about $4 \mu \mathrm{m}$, and stained with H\&E. Check the sample in the bright field with an Olympus BX-51 microscope. 


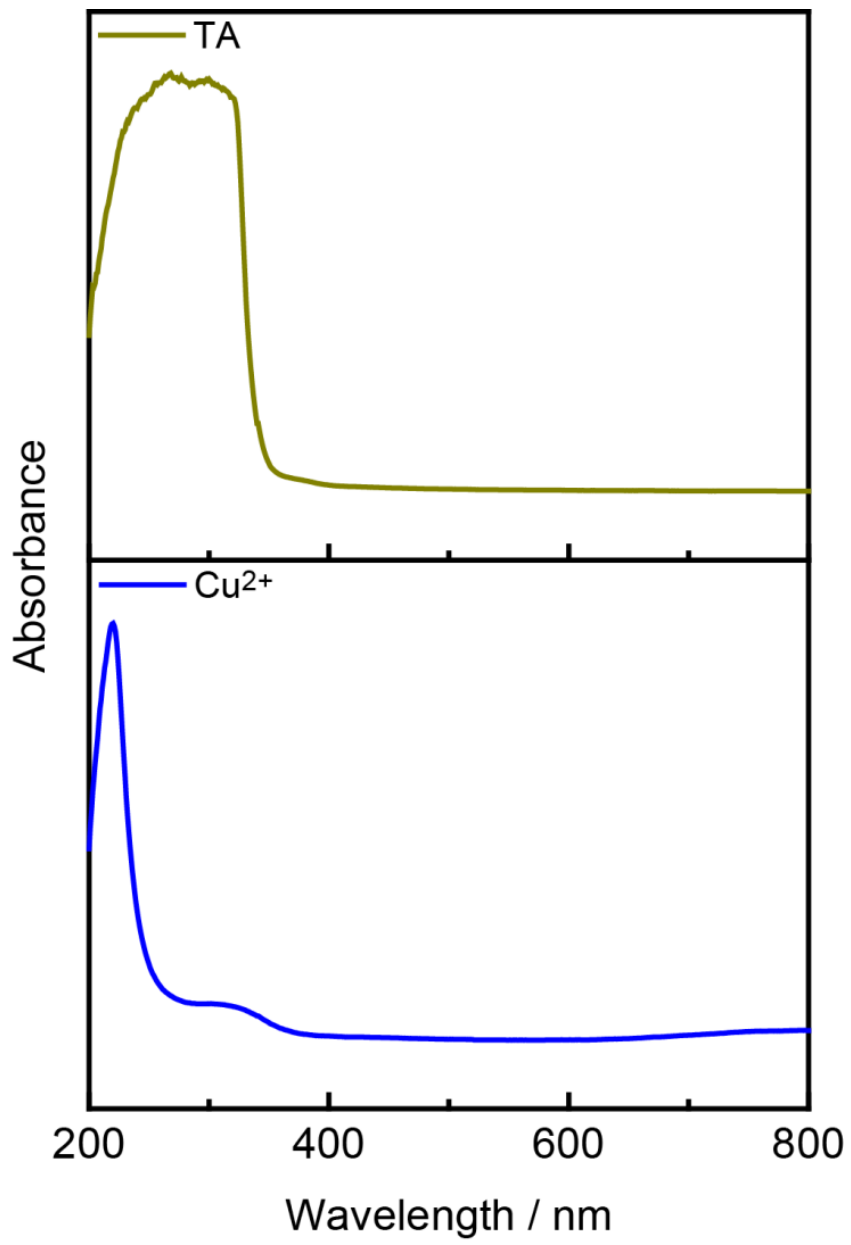

Figure S1. UV-Vis spectra of TA and $\mathrm{Cu}^{2+}$ solution. 


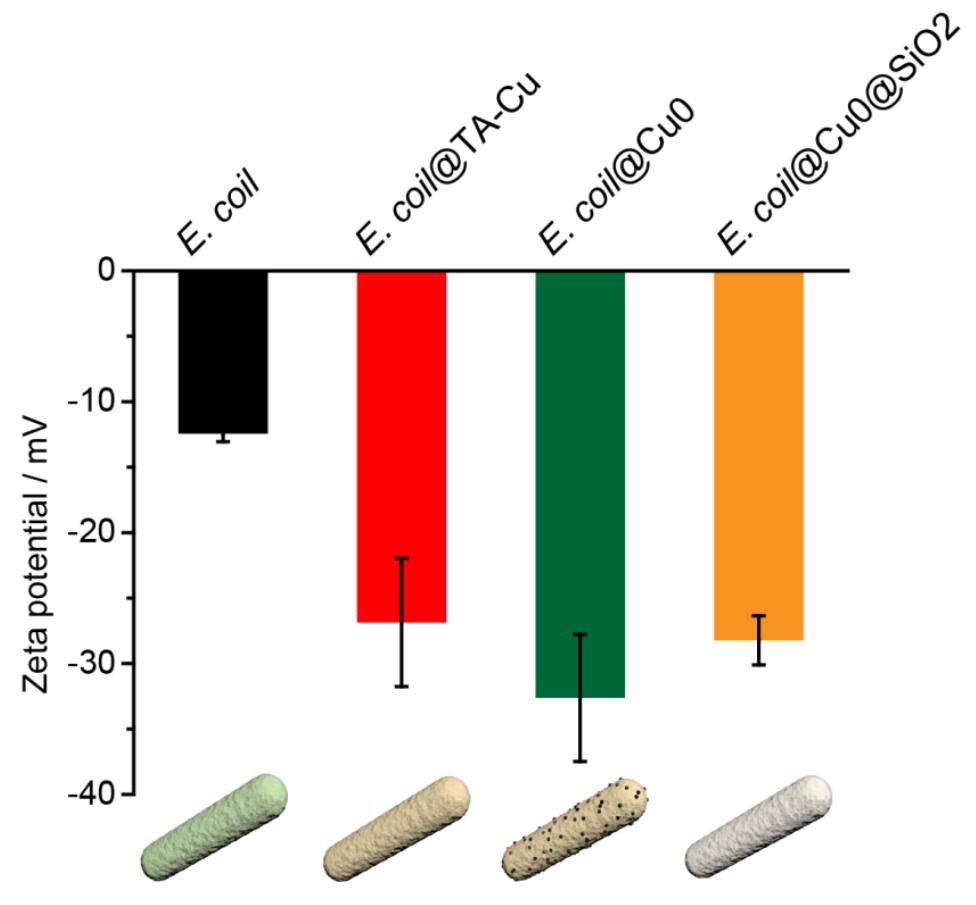

Figure S2. Zeta potentials of E.coli,E.coli@TA-Cu, E.coli@Cu0,E.coli@Cu0@SiO solution. 


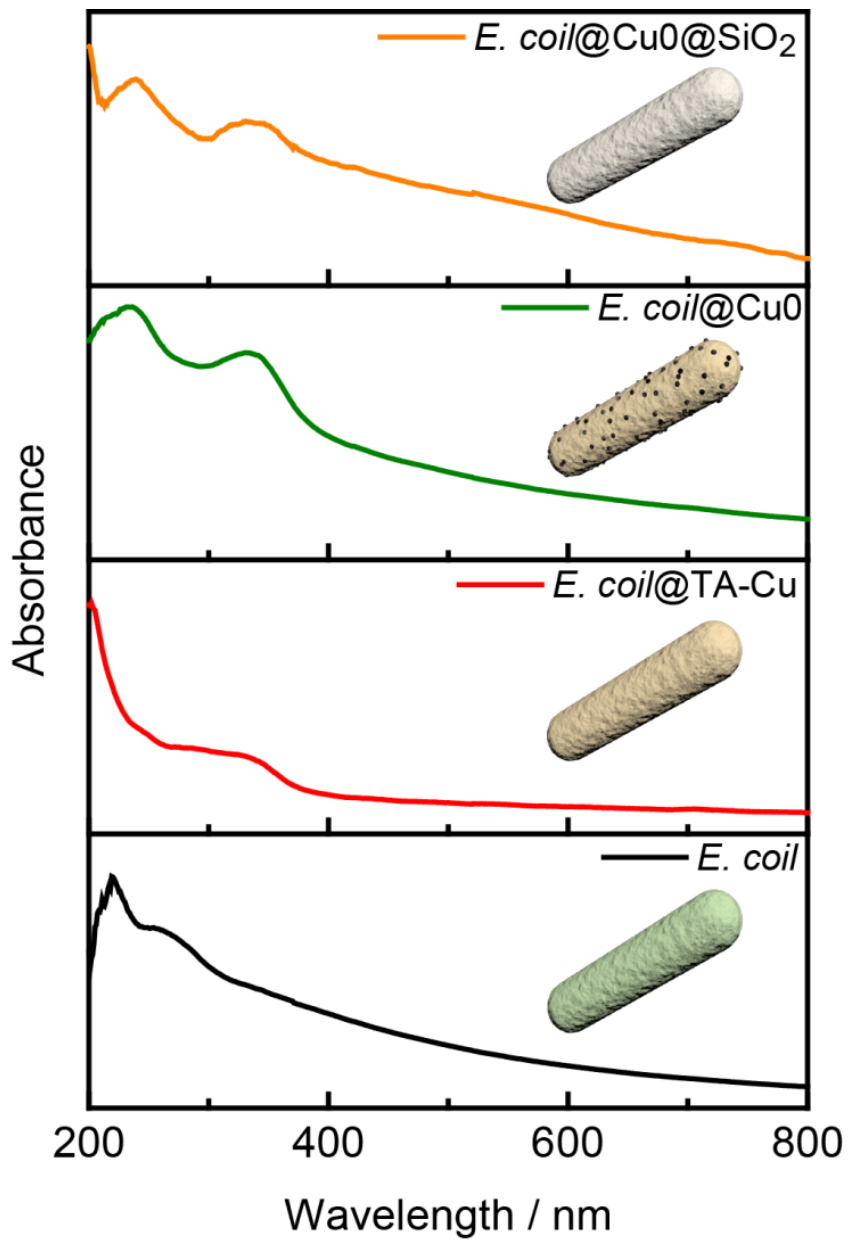

Figure S3. UV-Vis spectra of E.coli,E.coli@TA-Cu,E.coli@Cu0,E.coli@Cu0@SiO solution. 
a)
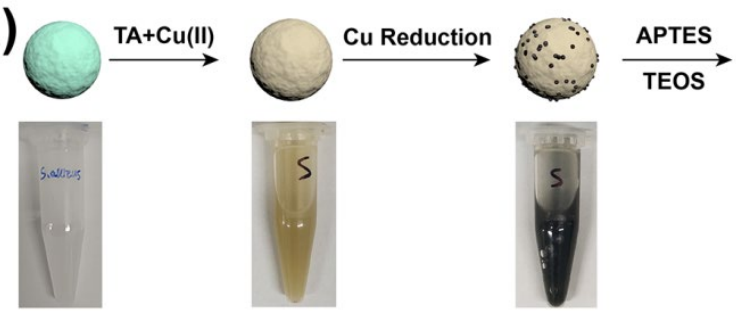

b)

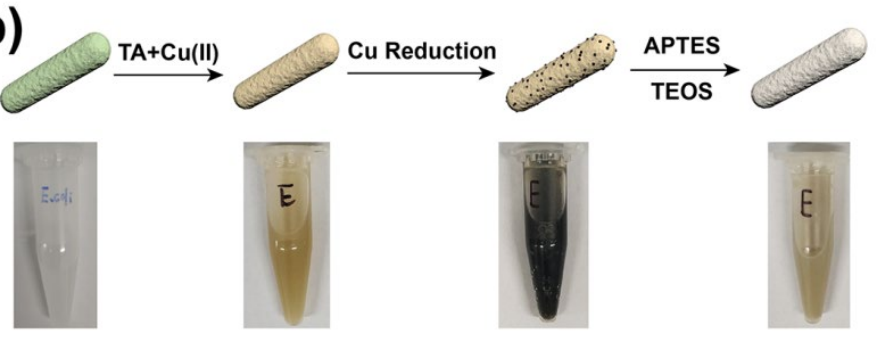

Figure S4. (a) Photo of S.aureus, S.aureus@TA-Cu, S.aureus@Cu ${ }^{0}, S$. aureus@Cu@ $@ \mathrm{SiO}_{2}$ solution. (b) Photo of E.coli,E.coli@TA-Cu,E.coli@Cu ${ }^{0}, E$.coli@Cu@ $\mathrm{SiO}_{2}$ solution. 


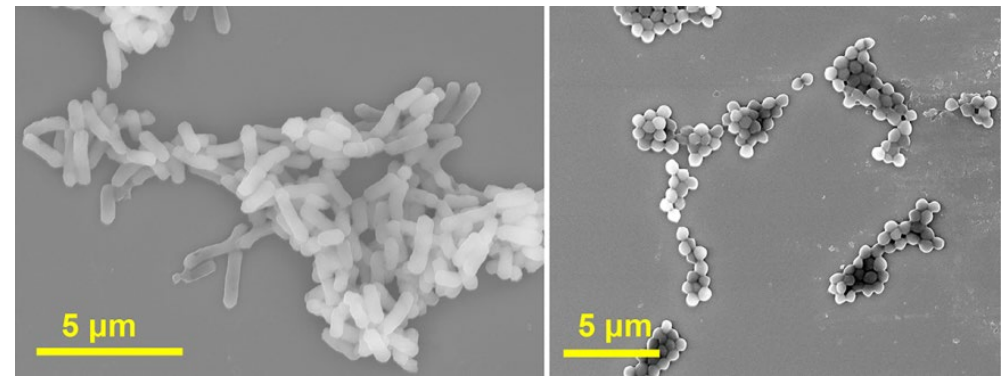

Figure S5. SEM images of E. coli and S. aureus. 

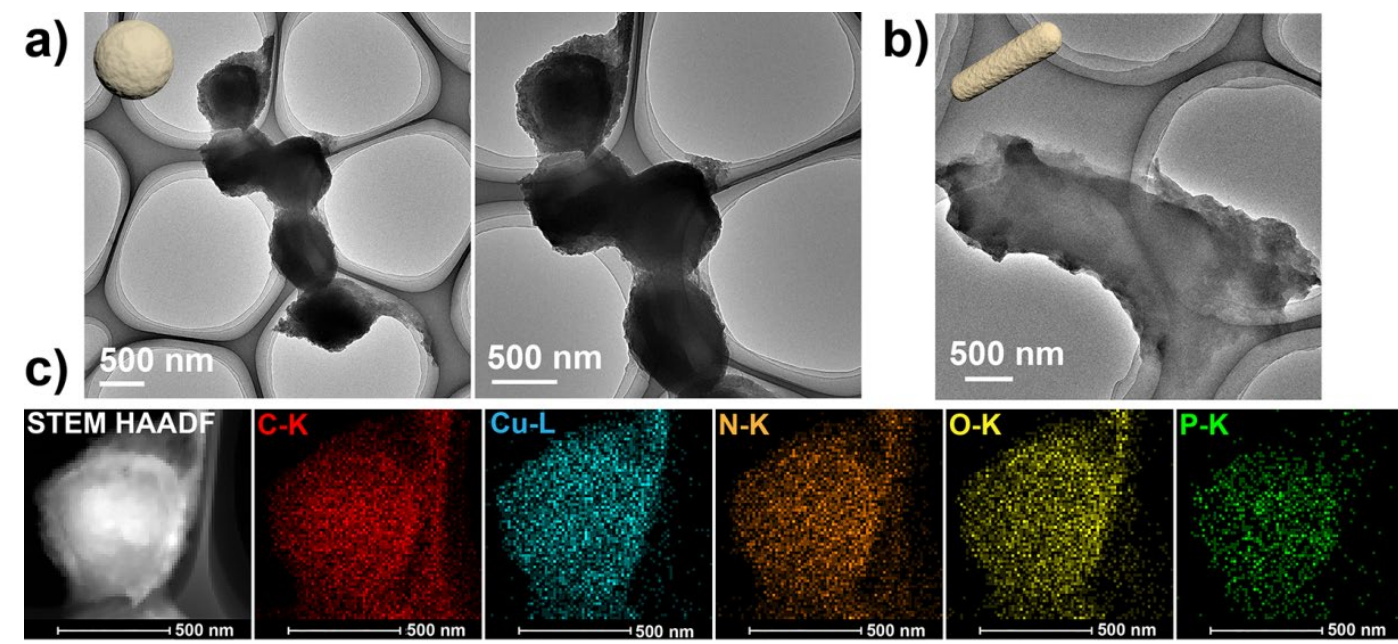

Figure S6. (a) TEM images of $S$. aureus@TA-Cu. (b) TEM images of E. coli@TA-Cu. (c)

Dark-field TEM images of S. aureus@TA-Cu and corresponding elemental mappings of C-K, $\mathrm{Cu}-\mathrm{L}, \mathrm{N}-\mathrm{K}, \mathrm{O}-\mathrm{K}$ and P-K signals.
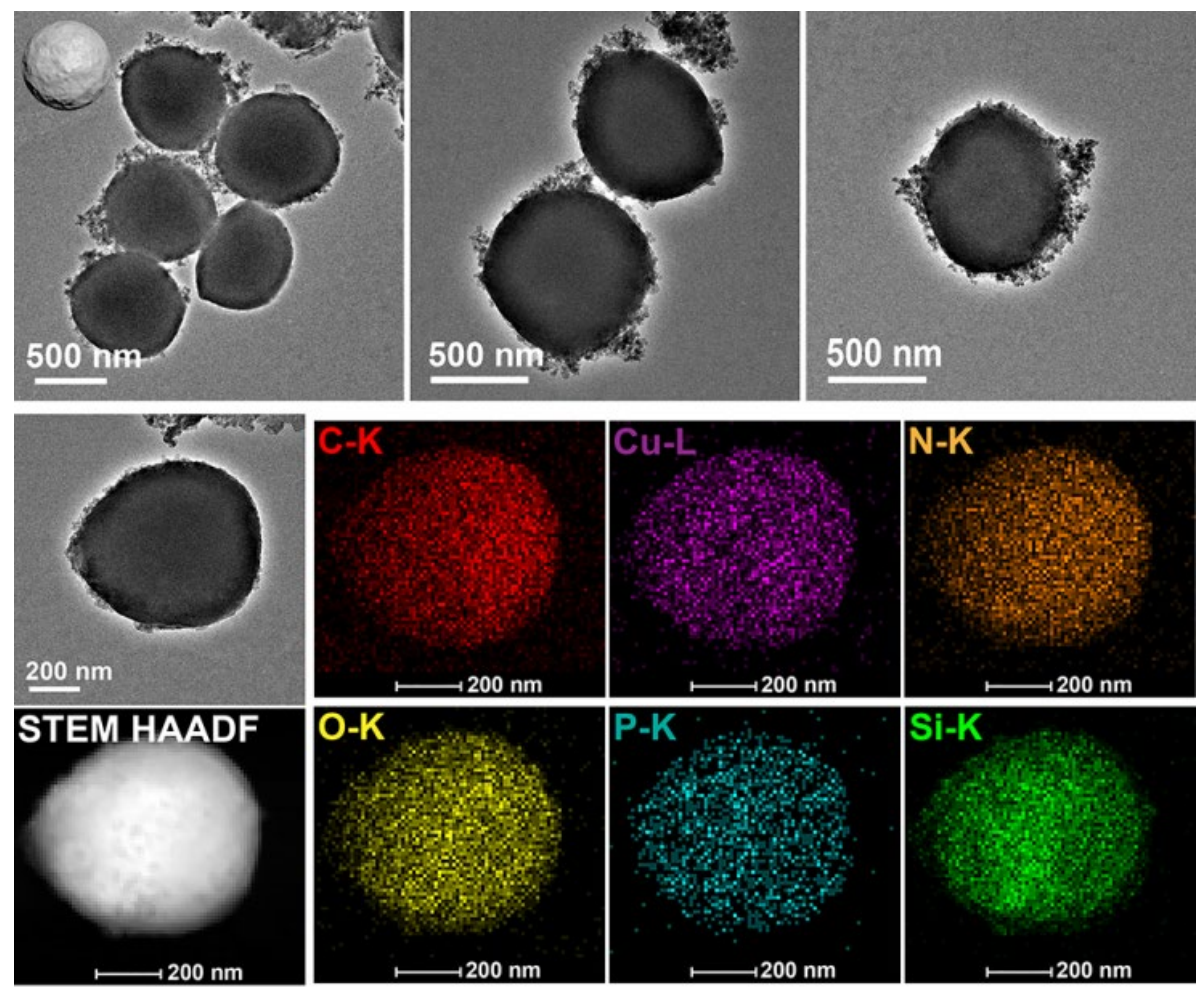

Figure S7. TEM images of $S$. aureus@ $\mathrm{Cu}^{0} @ \mathrm{SiO}_{2}$. And dark-field TEM images of $S$. aureus@Cu@ $@ \mathrm{SiO}_{2}$ and corresponding elemental mappings of C-K, Cu-L, N-K, O-K, P-K and Si-K signals. 

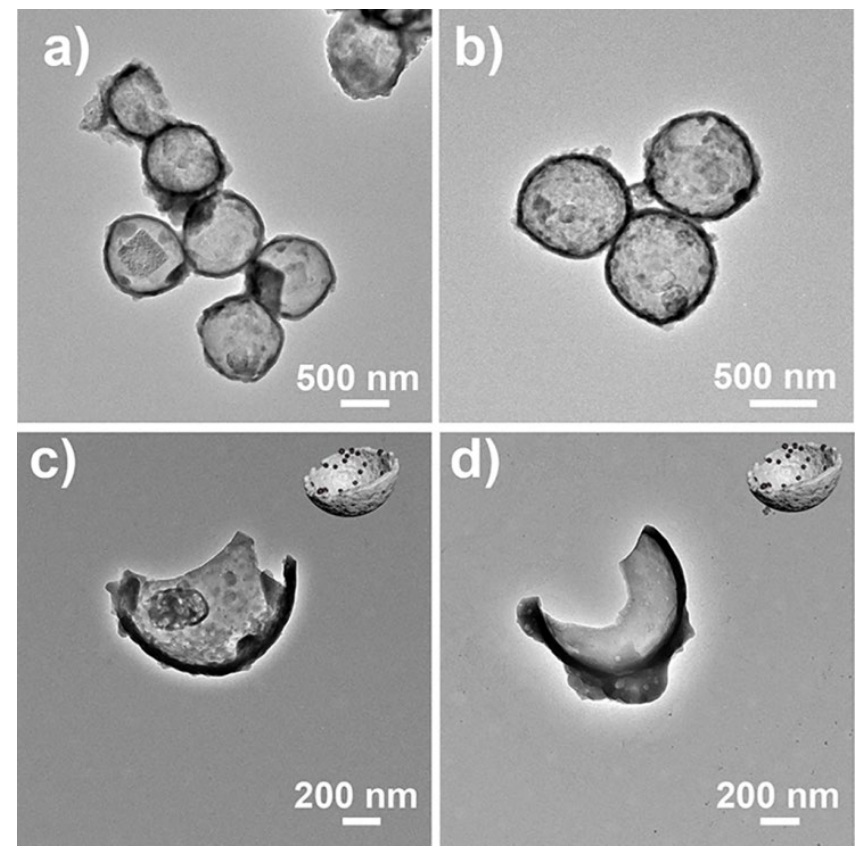

Figure S8. (a-b) TEM images of the obtained hollow $\mathrm{Cu} @ \mathrm{SiO}_{2}$ structure after removing the S. aureus template. (c-d) TEM images of the $\boldsymbol{S}$ - $\boldsymbol{A} \boldsymbol{b}$.
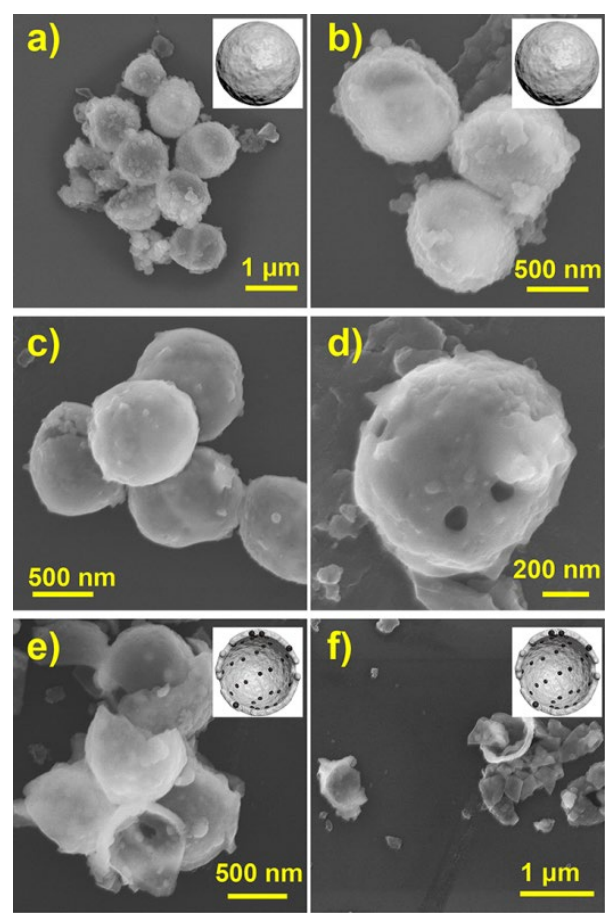

Figure S9. (a-b) SEM images of S.aureus@ $\mathrm{Cu}^{0} @ \mathrm{SiO}_{2}$. (c-d) SEM images of hollow $\mathrm{Cu} @ \mathrm{SiO}_{2}$ structure after removing the $S$. aureus template. (e-f) SEM images of $\boldsymbol{S}-\boldsymbol{A} \boldsymbol{b}$ after ultrasonic treatment. 

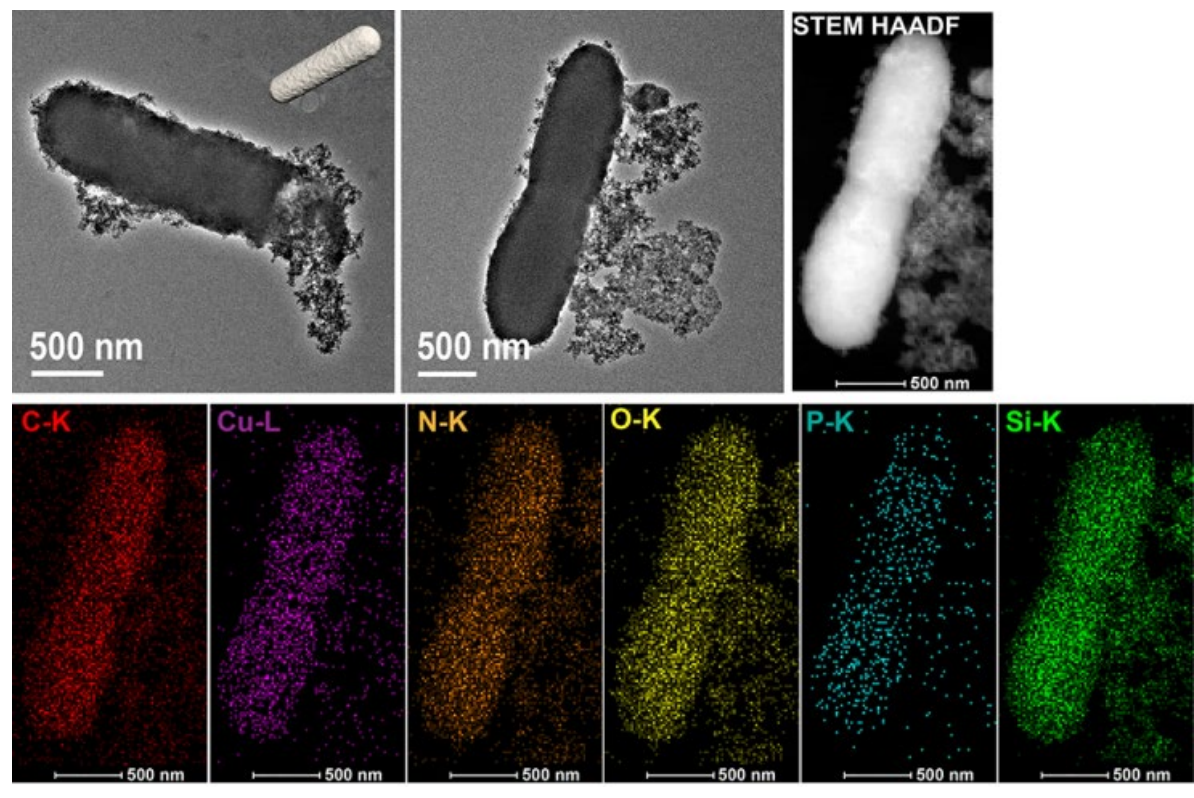

Figure S10. TEM images of E. coli@ $\mathrm{Cu}^{0} @ \mathrm{SiO}_{2}$. And dark-field TEM images of $E$. coli@ $\mathrm{Cu}^{0} @ \mathrm{SiO}_{2}$ and corresponding elemental mappings of C-K, Cu-L, N-K, O-K, P-K and Si-K signals.
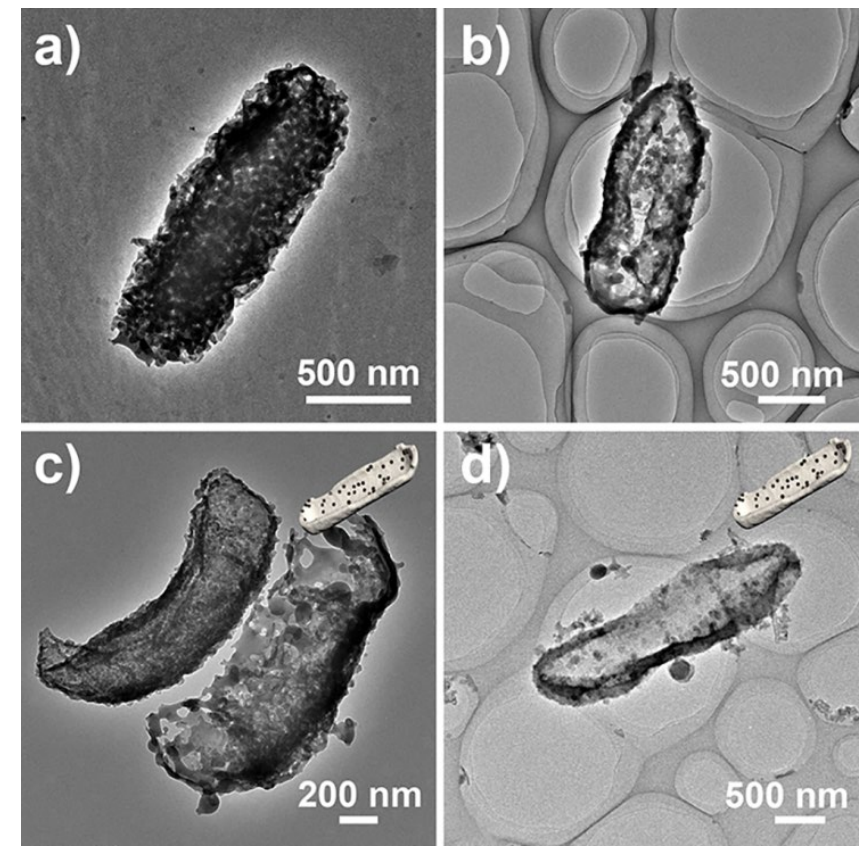

Figure S11. (a-b) TEM images of the obtained hollow $\mathrm{Cu} @ \mathrm{SiO}_{2}$ structure after removing the E. coli template. (c-d) TEM images of the $\boldsymbol{E}-\boldsymbol{A} \boldsymbol{b}$. 


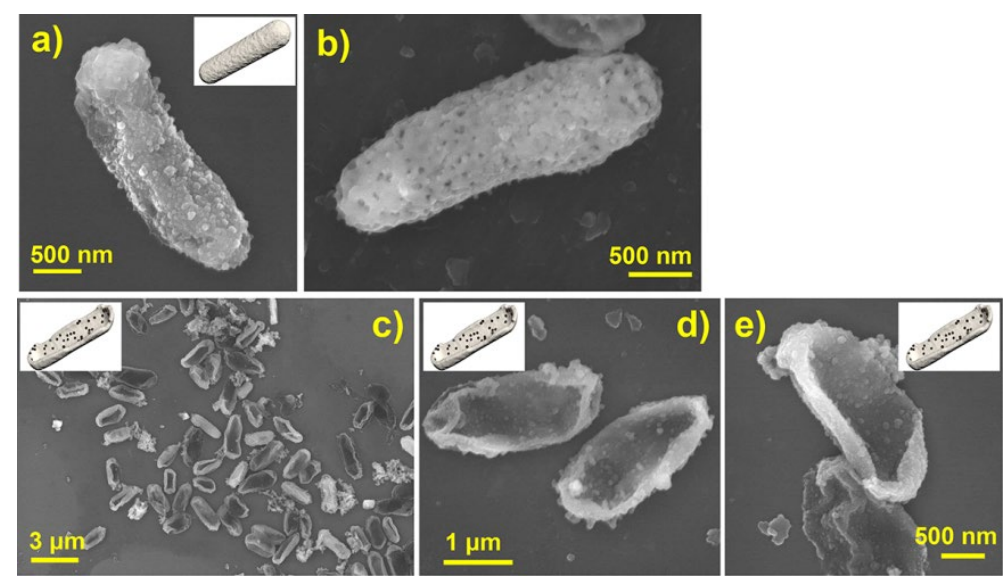

Figure S12. (a) SEM image of E.coli@ $\mathrm{Cu}^{0} @ \mathrm{SiO}_{2}$. (b) SEM image of hollow $\mathrm{Cu} @ \mathrm{SiO}_{2}$ structure after removing the $E$. coli template. (c-e) SEM images of $\boldsymbol{E}-\boldsymbol{A} \boldsymbol{b}$ after ultrasonic treatment. 

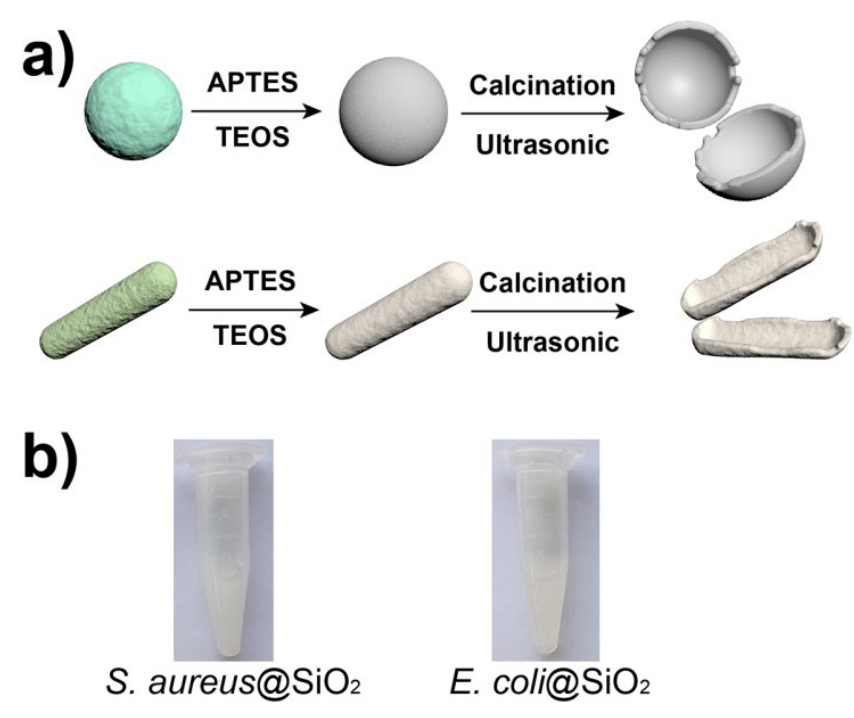

Figure S13. (a) Copper-free bacterial imprinting shell construction process. (b) Photo of $S$. aureus@ $\mathrm{SiO}_{2}$ solution, and E.coli@ $\mathrm{SiO}_{2}$ solution. 

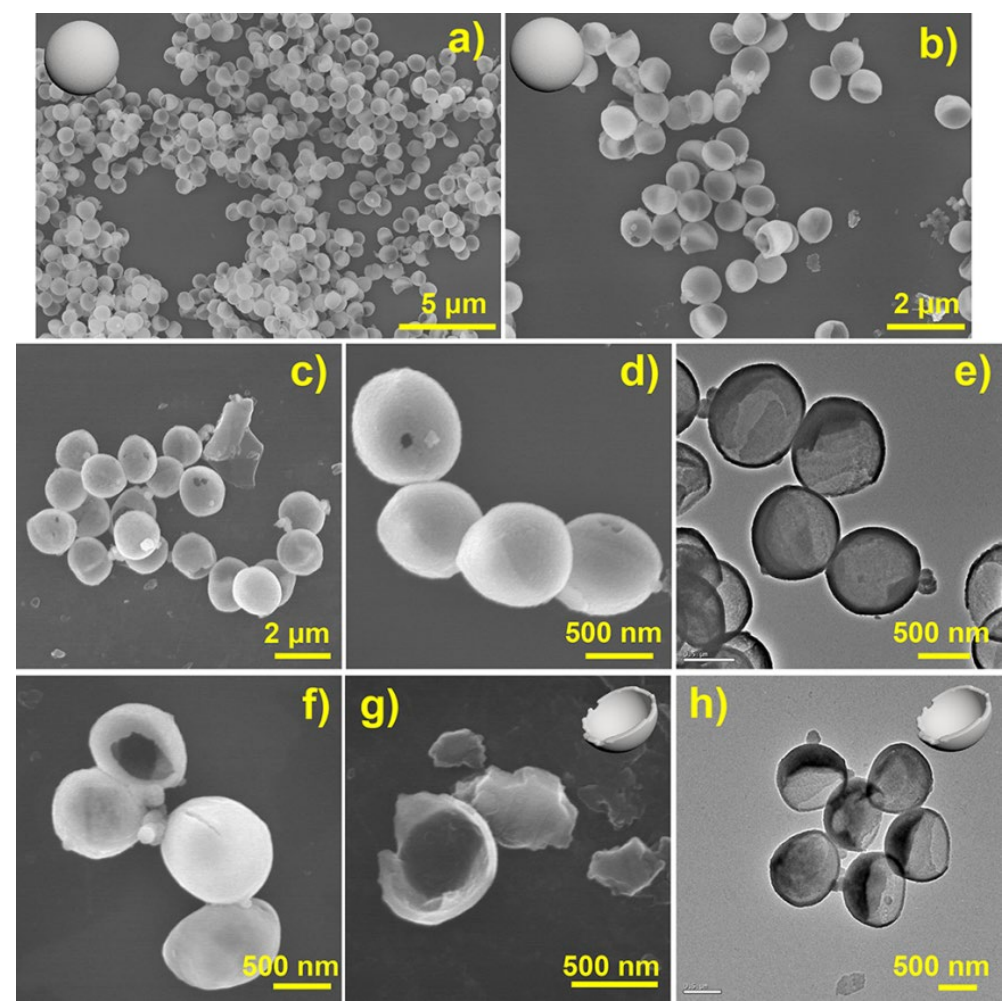

Figure S14. (a-b) SEM images of Copper-free S. aureus@ $\mathrm{SiO}_{2}$. (c-d) SEM images of hollow $\mathrm{SiO}_{2}$ structure after removing the $S$. aureus template. (e) TEM images of hollow $\mathrm{SiO}_{2}$ structure. (f) SEM images of hollow $\mathrm{SiO}_{2}$ structure after ultrasonic treatment for a short time. (g-h) SEM and TEM images of half-shell fragment $\mathrm{SiO}_{2}$ structure after ultrasonic treatment. 


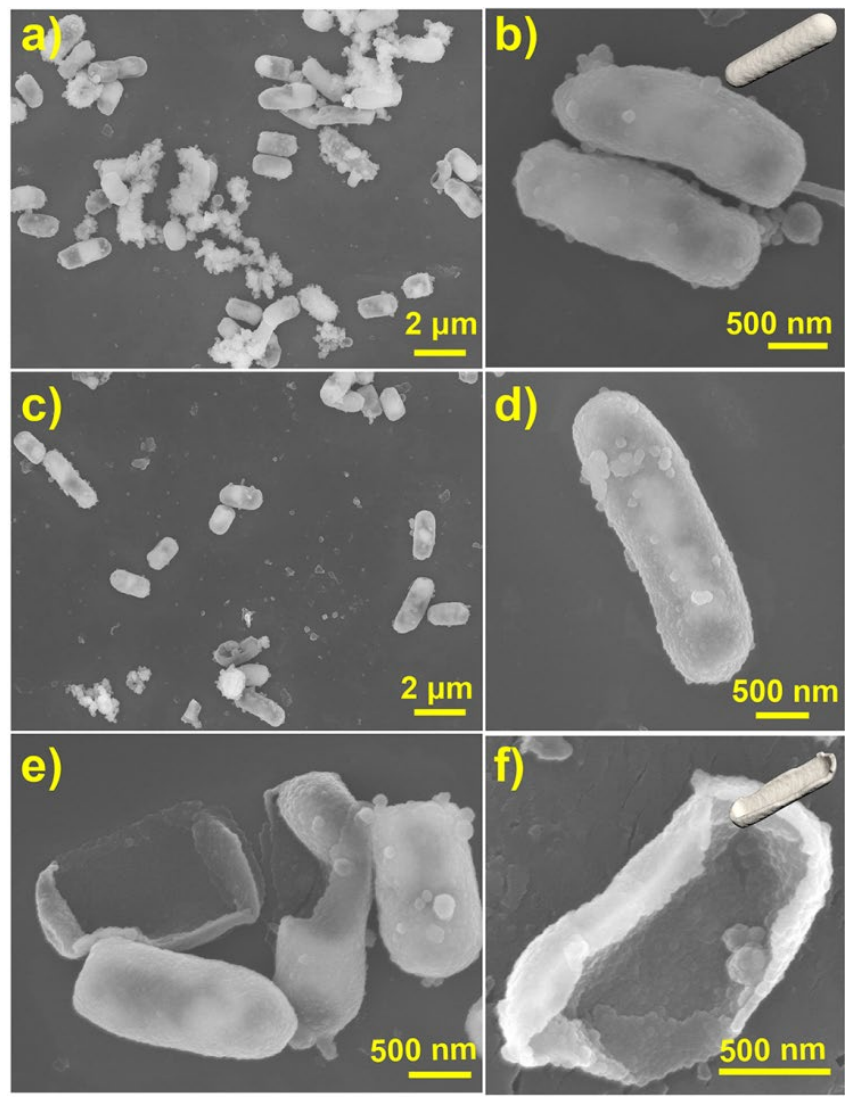

Figure S15. (a-b) SEM images of Copper-free E. coli@SiO 2 . (c-d) SEM images of the obtained hollow $\mathrm{SiO}_{2}$ structure after removing the E. coli template. (e-f) SEM images of half-shell fragment $\mathrm{SiO}_{2}$ structure after ultrasonic treatment. 
Click reaction of 3-azido-7-hydroxycoumarin $\mathbf{1}$ and phenylacetylene $\mathbf{2}$ was carried out to evaluate the catalytic efficiency of $\boldsymbol{E}-\boldsymbol{A} \boldsymbol{b}$ or $\boldsymbol{S}-\boldsymbol{A} \boldsymbol{b}$. Mixed $\boldsymbol{E}-\boldsymbol{A} \boldsymbol{b}$ or $\boldsymbol{S}-\boldsymbol{A b}(100 \mu \mathrm{g} / \mathrm{mL}), 80 \mu \mathrm{M}$ 3-azido-7-hydroxycoumarin (4 mM in DMSO) and phenylacetylene in water at $37^{\circ} \mathrm{C}$. After a period of time, the mixture was centrifuged to remove the catalyst. The fluorescence change of the supernatant was measured with a fluorescence spectrometer. The fluorescence performance of $\mathbf{3}$ is also shown in Figure $\mathbf{S 1 8 .}$

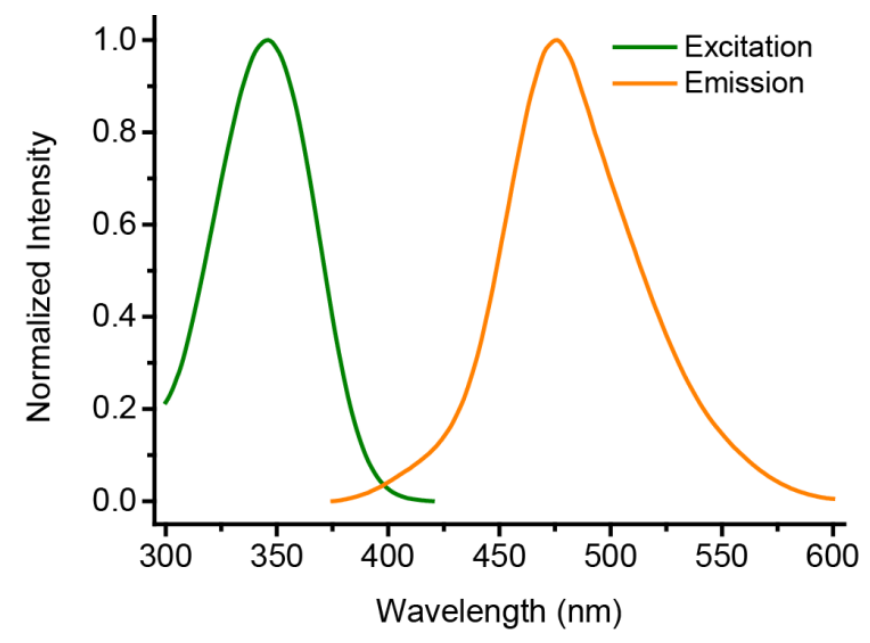

Figure S16. Normalized excitation and emission of $\mathbf{3}$ in $\mathrm{H}_{2} \mathrm{O}$. Cycloaddition of $\mathbf{1}$ and $\mathbf{2}$ afforded $\mathbf{3}\left(\lambda_{\mathrm{ex}}, 350 \mathrm{~nm}\right.$ and $\left.\lambda_{\mathrm{em}}, 475 \mathrm{~nm}\right)$. 


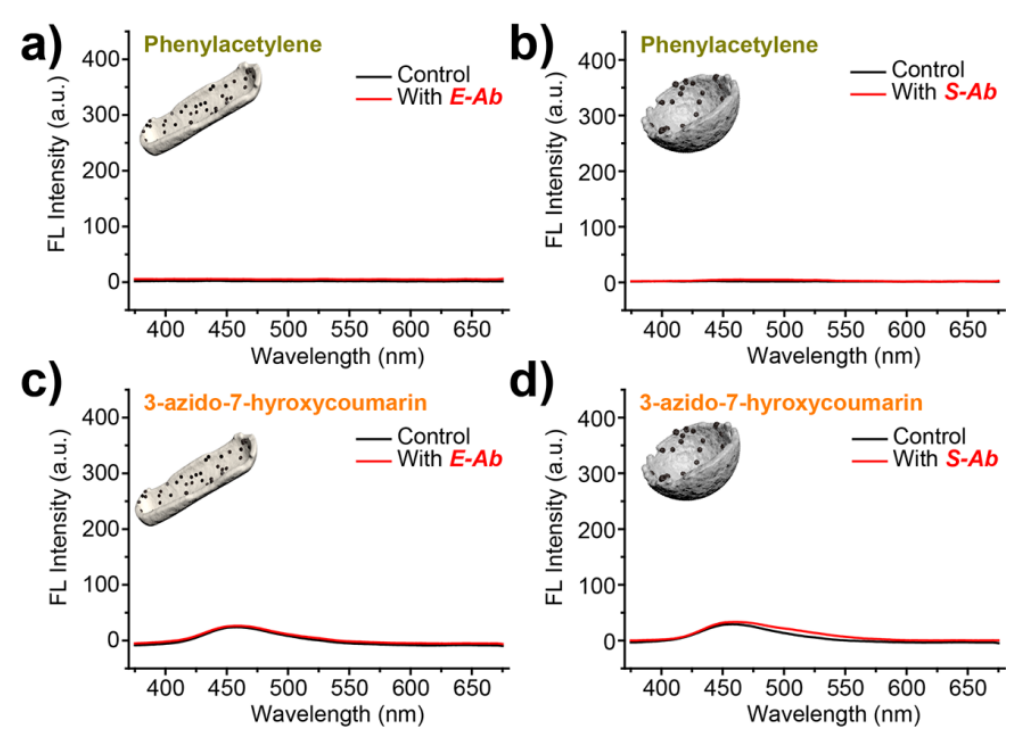

Figure S17. (a), (b) Fluorescence emission spectra of phenylacetylene mixed with $\boldsymbol{E}-\boldsymbol{A} \boldsymbol{b}$ or

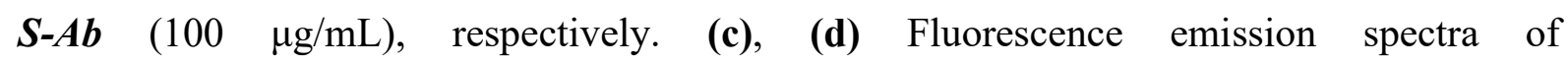
3-azido-7-hydroxycoumarin mixed with $\boldsymbol{E}-\boldsymbol{A} \boldsymbol{b}$ or $\boldsymbol{S}-\boldsymbol{A} \boldsymbol{b}$, respectively. The results showed that 3-azido-7-hydroxycoumarin had weak fluorescence, while phenylacetylene itself had no fluorescence. And the catalysts had no effects in fluorescence of 3-azido-7-hydroxycoumarin and phenylacetylene alone. 


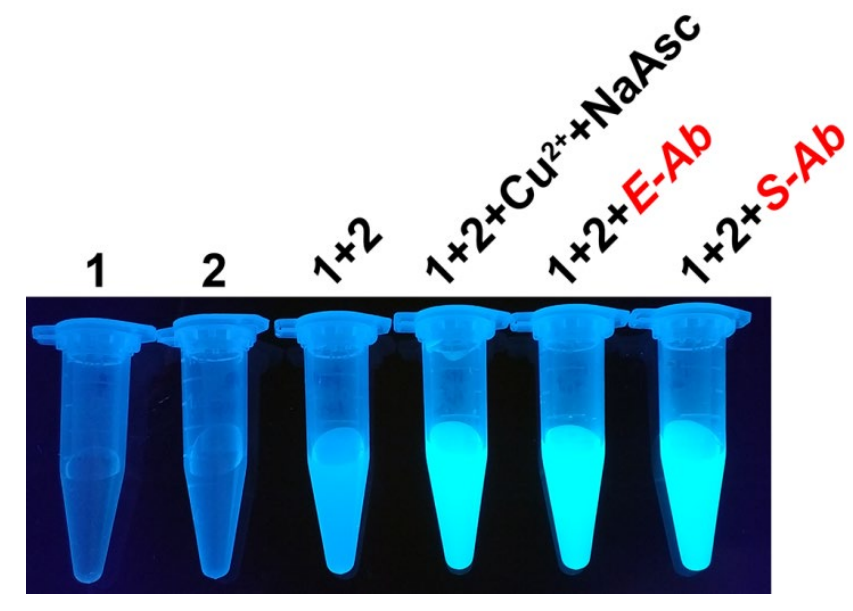

Figure S18. Fluorescence photographs of precursor molecules 1, 2 treated with different catalysts $\left(\mathrm{Cu}^{2+}=50 \mu \mathrm{M}, \mathrm{NaAsc}=100 \mu \mathrm{M} ; \boldsymbol{E}-\boldsymbol{A b} / \boldsymbol{S}-\boldsymbol{A b}(100 \mu \mathrm{g} / \mathrm{mL})\right)$ in $\mathrm{H}_{2} \mathrm{O} / \mathrm{DMSO}(9: 1)$. The results indicated that $\boldsymbol{E}-\boldsymbol{A} \boldsymbol{b}$ and $\boldsymbol{S}-\boldsymbol{A} \boldsymbol{b}$ can catalyze $\mathbf{1}$ and $\mathbf{2}$ to produce fluorescent molecule 3 .

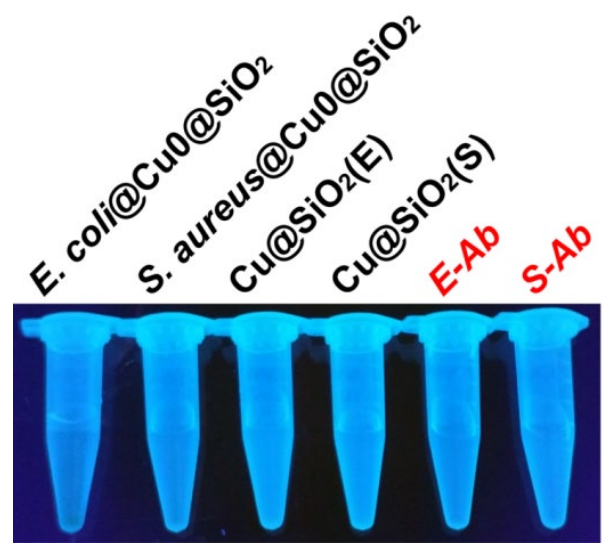

Figure S19. Fluorescence photos of different materials. The results indicated that none of the materials had auto-fluorescence. 
Synthesis of precursor chemical substrate molecules in this study according to previous report. $^{1-3}$

Synthetic route of precursor molecules 4,5 and compound 6
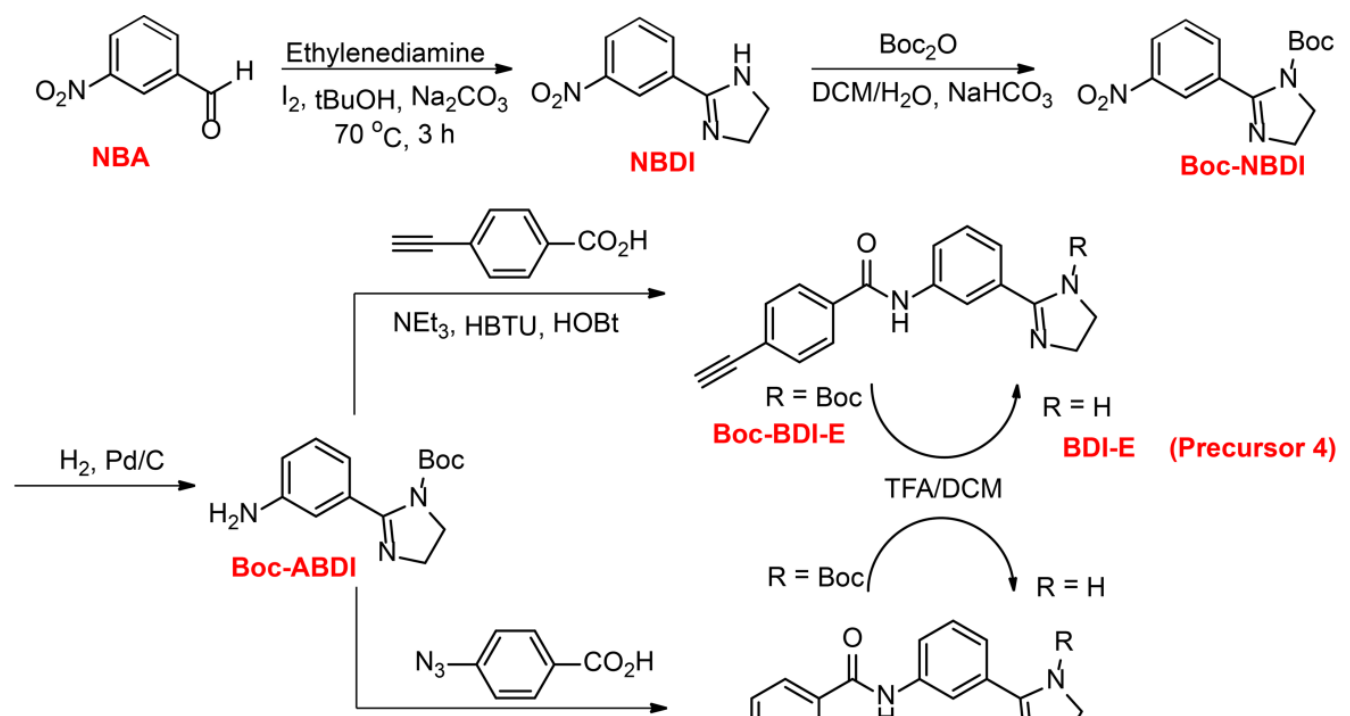

$\mathrm{NEt}_{3}, \mathrm{HBTU}, \mathrm{HOBt}$
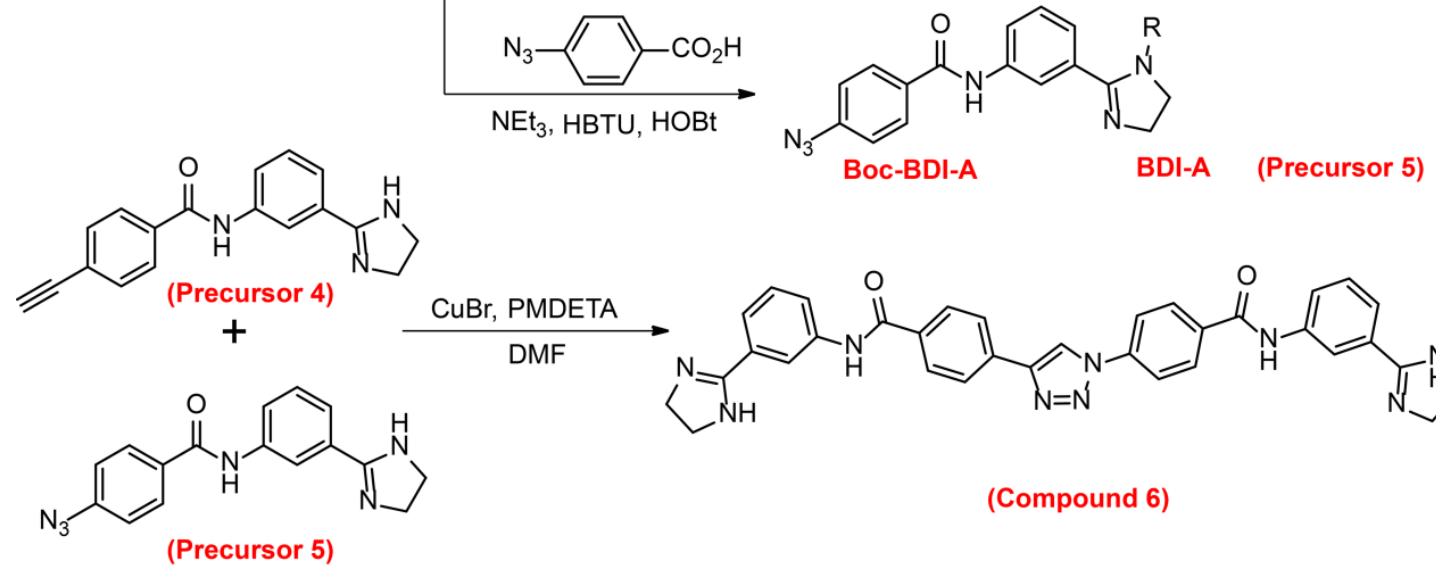

$N$-boc-2-(3-nitrophenyl)-4,5-dihydro-1 $H$-imidazole (Boc-NBDI) synthesis process.

Combined 3-nitrobenzaldehyde $(3.02 \mathrm{~g}, 20 \mathrm{mmol})$ and ethylenediamine $(1.47 \mathrm{~mL}, 22 \mathrm{mmol})$ in tert-butanol $(100 \mathrm{~mL})$, and stirred the mixture for 30 minutes. $\mathrm{Na}_{2} \mathrm{CO}_{3}(6.36 \mathrm{~g}, 60 \mathrm{mmol})$ and $\mathrm{I}_{2}(6.34 \mathrm{~g}, 25 \mathrm{mmol})$ were added to the mixture, and then the reaction temperature was increased to $70{ }^{\circ} \mathrm{C}$ for $3 \mathrm{~h}$. The solution was cooled, and saturated aqueous $\mathrm{Na}_{2} \mathrm{SO}_{3}$ was added to quench the excess $\mathrm{I}_{2}$ to obtain a pale yellow mixture. Removed solvent under reduced pressure. $100 \mathrm{~mL}$ Ethyl acetate was added to the mixture, sonicated and filtered to remove insoluble materials. Washed the solution with saturated aqueous $\mathrm{Na}_{2} \mathrm{SO}_{3}(100 \mathrm{~mL})$, 
brine $(100 \mathrm{~mL})$, and dried over $\mathrm{Na}_{2} \mathrm{SO}_{4}$. Removed the ethyl acetate to give a pale yellow solid under reduced pressure, and redissolved in $50 \mathrm{~mL}$ DCM. The DCM solution was cooled to $0^{\circ} \mathrm{C}$, and $\mathrm{Boc}_{2} \mathrm{O}(6.6 \mathrm{~g}$, excess) was added to the solution together with $50 \mathrm{~mL}$ aqueous $\mathrm{NaHCO}_{3}$. The two-layer mixture was gradually warmed to room temperature, stirred overnight. The DCM layer was separated, dried with $\mathrm{Na}_{2} \mathrm{SO}_{4}$ and evaporated to give a crude product, which was further purified by column chromatography (silica, ethyl acetate/hexane $1: 2$ to $1: 1)$ to give a pale yellow oily product.

$N$-boc-2-(3-aminophenyl)-4,5-dihydro-1H-imidazole (Boc-ABDI) synthesis process.

Dissolved the nitro product $N$-Boc-2-(3-aminophenyl)-4,5-dihydro- $1 H$-imidazole (1.2 g, 4.1 mmol) from the previous step in $20 \mathrm{~mL}$ THF or ethyl acetate. $150 \mathrm{mg} 10 \% \mathrm{Pd} / \mathrm{C}$ was added. Reaction vessel was deoxygenated and added hydrogen via a balloon. Reaction was overnight at room temperature. Resulting mixture was filtered and evaporated and then obtained the pure product as a light white solid.

Synthesis process of compound $4(\mathrm{BDI}-\mathrm{E}) \cdot \mathrm{TFA}$.

Mixed $N$-Boc-2-(3-aminophenyl)-4,5-dihydro- $1 H$-imidazole (previous step) (0.78 g, 3.0 mmol) and 4-alkynylbenzoic acid (0.54 g, $3.3 \mathrm{mmol})$, HBTU (1.37) g, $3.6 \mathrm{mmol})$, HOBt $(0.36 \mathrm{~g}, 2.7 \mathrm{mmol})$ in $15 \mathrm{~mL} \mathrm{DMF}$. Added triethylamine $(0.84 \mathrm{~mL}, 6.0 \mathrm{mmol})$ to the solution, and kept the reaction at room temperature overnight. Poured the solution into $125 \mathrm{~mL}$ water and stirred for 5 minutes, then filtered to collect the precipitate. Redissolved the solid in 50 $\mathrm{mL}$ ethyl acetate and washed twice with aqueous $\mathrm{NaOH}(1 \mathrm{M}, 50 \mathrm{~mL})$ and once with $50 \mathrm{~mL}$ brine. Dried the solution over $\mathrm{Na}_{2} \mathrm{SO}_{4}$ and evaporated to give the crude, Boc-protected product, and purified by column chromatography (silica, ethyl acetate/hexane 1:2 to 1:1). The 
purified product was deprotected with TFA $(50 \% \mathrm{v} / \mathrm{v}$ in $\mathrm{DCM}, 10 \mathrm{~mL})$ for 3 hours at $0^{\circ} \mathrm{C}$. The TFA and DCM were removed under reduced pressure. Washed the residue was with ether and dried to obtain the product in the form of the TFA salt as a pale yellow solid.

Synthesis process of compound 5 (BDI-A)·TFA.

The synthesis of $\mathbf{5}$.TFA was similar to the synthesis of $4 \cdot$ TFA, using $N$-Boc-2-(3-aminophenyl)-4,5-dihydro-1H-imidazole and replaced 4-alkynylbenzoic acid with 4-azidobenzoic acid.

Synthesis of compound $6 \cdot$ TFA.

Compound 4 • TFA $(12.1 \mathrm{mg}, 30 \mu \mathrm{mol}), \mathbf{5}$ - TFA $(12.6 \mathrm{mg}, 30 \mu \mathrm{mol})$ and $\mathrm{CuBr}(1.1 \mathrm{mg}, 7.5$ $\mu \mathrm{mol})$ was dissolved in DMF $(0.5 \mathrm{~mL})$ in a $1.5 \mathrm{~mL}$ vial equipped with a Teflon cap. The vial was deoxygenated thoroughly. PMDETA $(10 \mu \mathrm{L})$ was added into the vial under $\mathrm{N} 2$ protection, and the mixture turned into a yellowish-green solution. The solution was stirred at room temperature overnight, and a white precipitate gradually formed. The white precipitate was collected by centrifugation, washed with DMF $(0.5 \mathrm{~mL})$ once, and with diethyl ether $(0.5$ $\mathrm{mL}$ ) three times. The solid was dried under reduced pressure to give pure $6 \cdot$ TFA as an almost-white solid. 

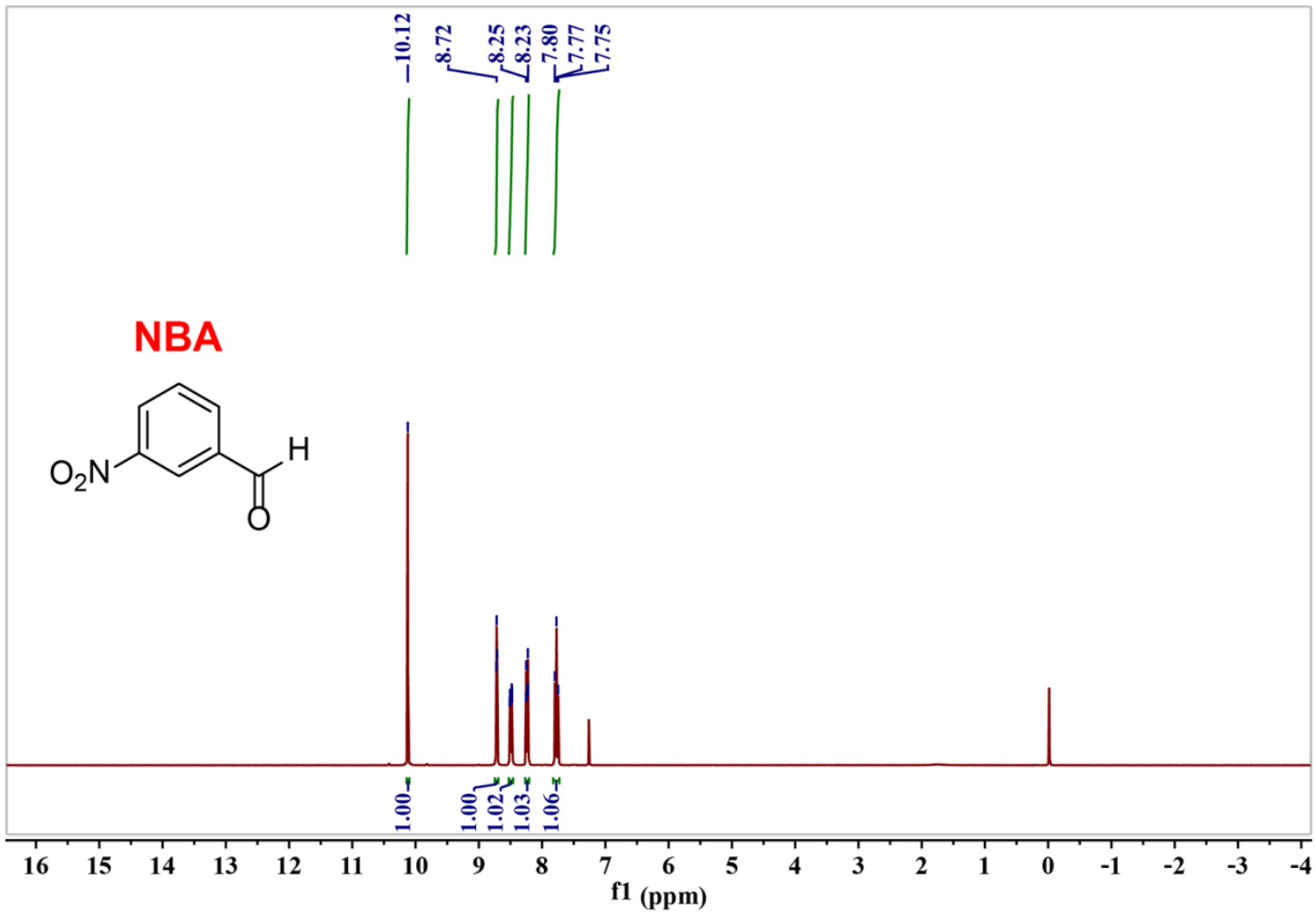

Figure S20. The ${ }^{1} \mathrm{H}$ NMR spectrum of 3-Nitrobenzaldehyde (NBA) in $\mathrm{CDCl}_{3}$.

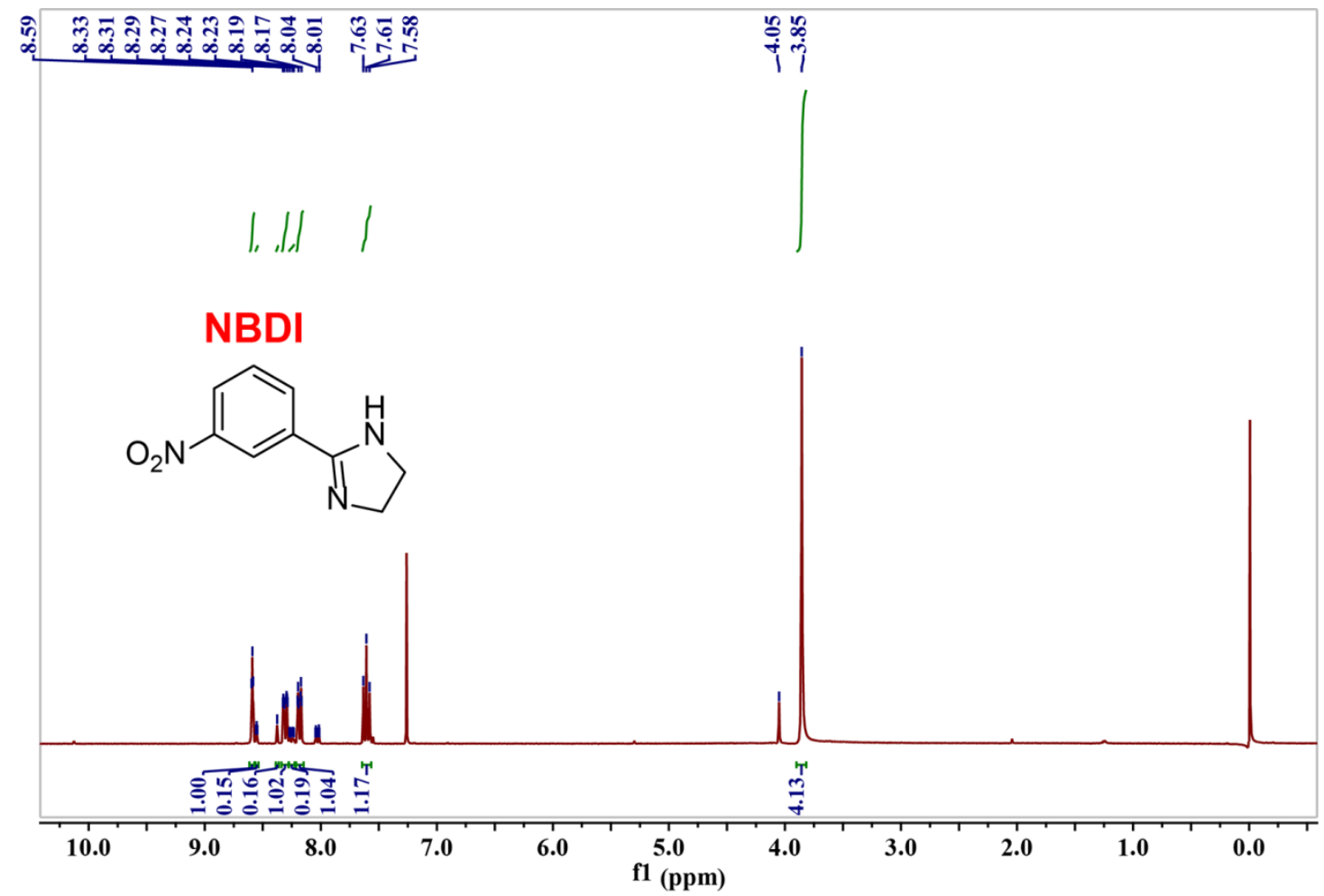

Figure S21. The ${ }^{1} \mathrm{H}$ NMR spectrum of 2-(3-nitrophenyl)-4,5-dihydro-1H-imidazole (NBDI) in $\mathrm{CDCl}_{3}$. 


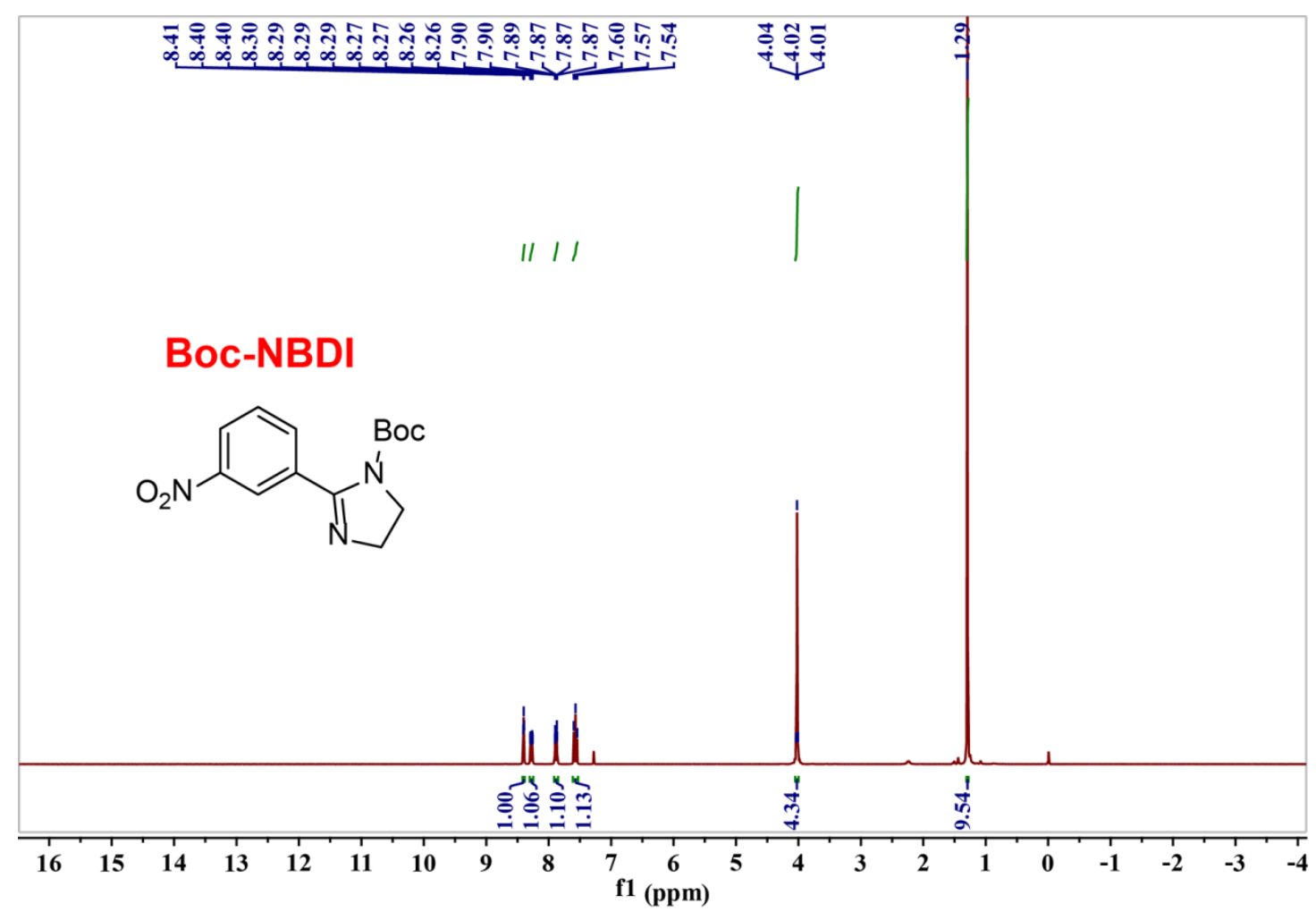

Figure S22. The ${ }^{1} \mathrm{H}$ NMR spectrum of $N$-boc-2-(3-nitrophenyl)-4,5-dihydro-1 $H$-imidazole (Boc-NBDI) in $\mathrm{CDCl}_{3}$.

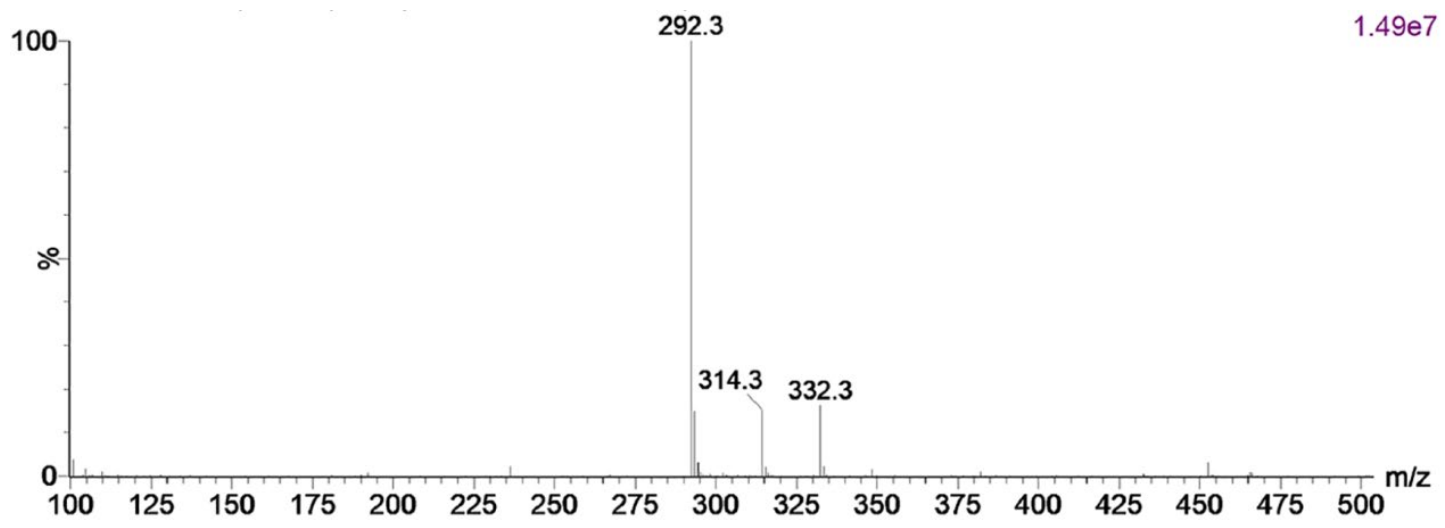

Figure S23. The MS analysis confirming the successful synthesis of Boc-NBDI. 


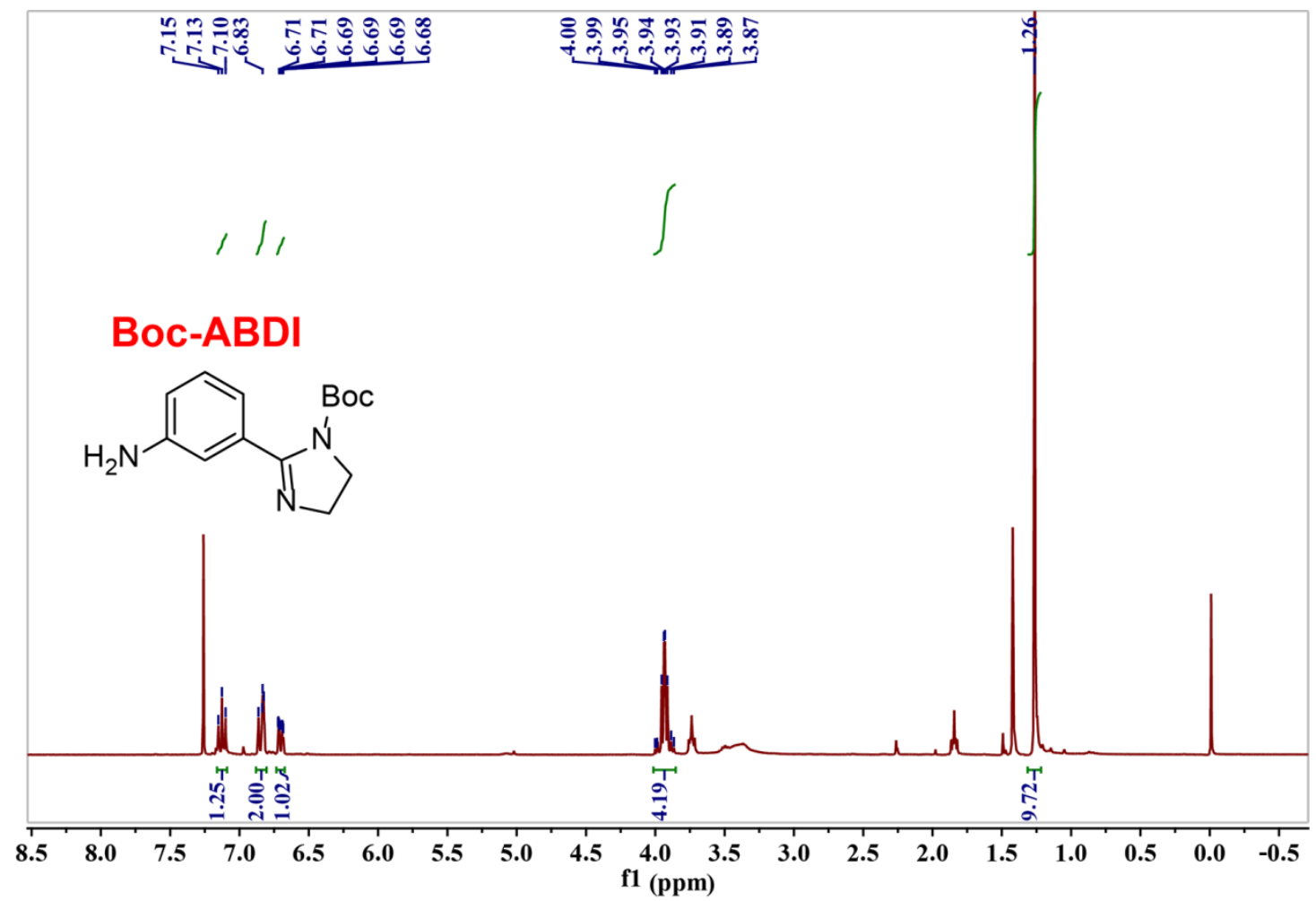

Figure S24. The ${ }^{1} \mathrm{H}$ NMR spectrum of $N$-boc-2-(3-aminophenyl)-4,5-dihydro- $1 H$-imidazole (Boc-ABDI) in $\mathrm{CDCl}_{3}$. 


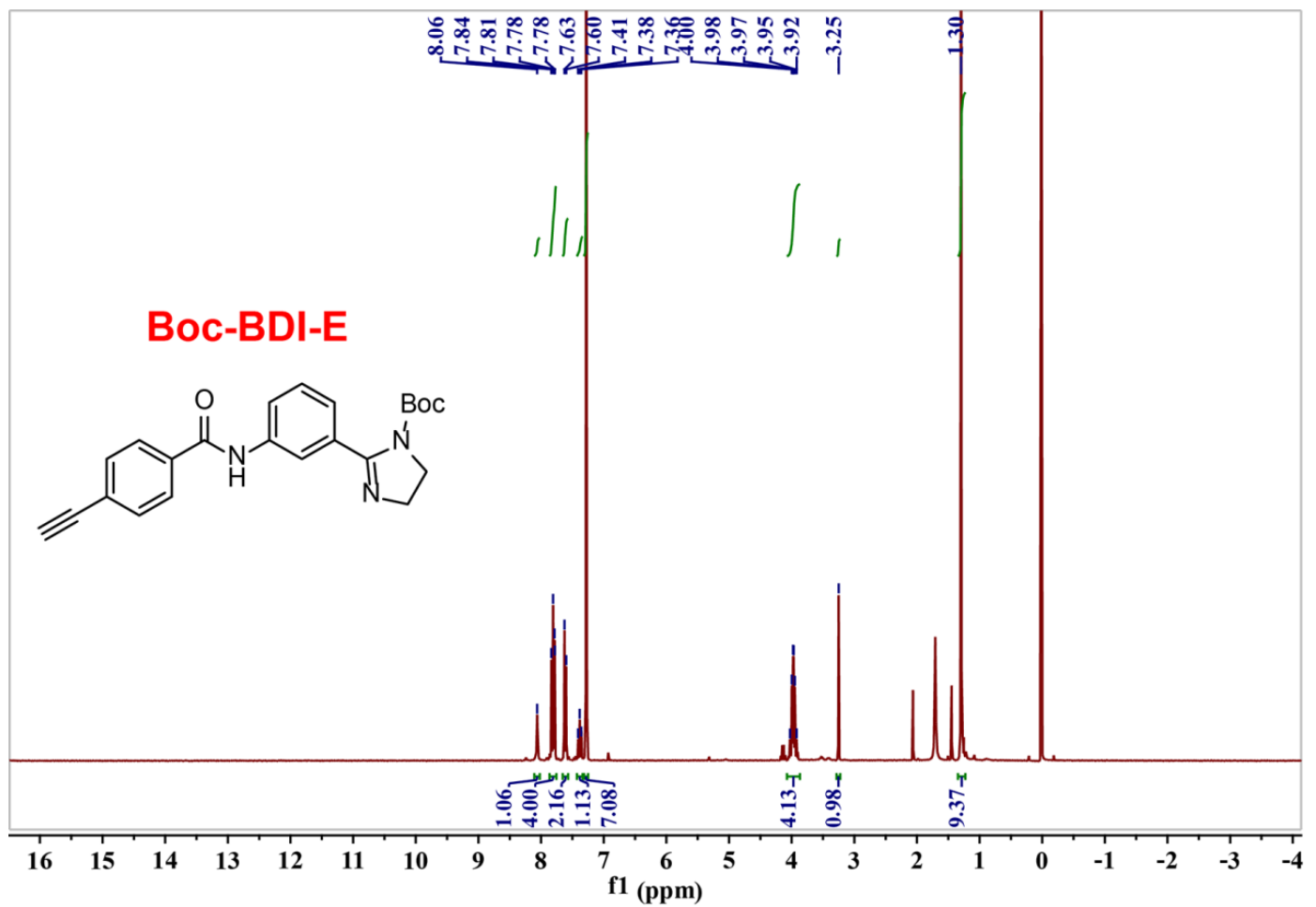

$\begin{array}{llllll}\text { Figure } & \text { S25. } & \text { The } & { }^{1} \mathrm{H} & \mathrm{NMR} & \text { spectrum }\end{array}$

$\mathrm{N}$-boc-2-(3-(4-ethynylbenzamido)phenyl)-4,5-dihydro-1H-imidazole (Boc-BDI-E) in $\mathrm{CDCl}_{3}$.

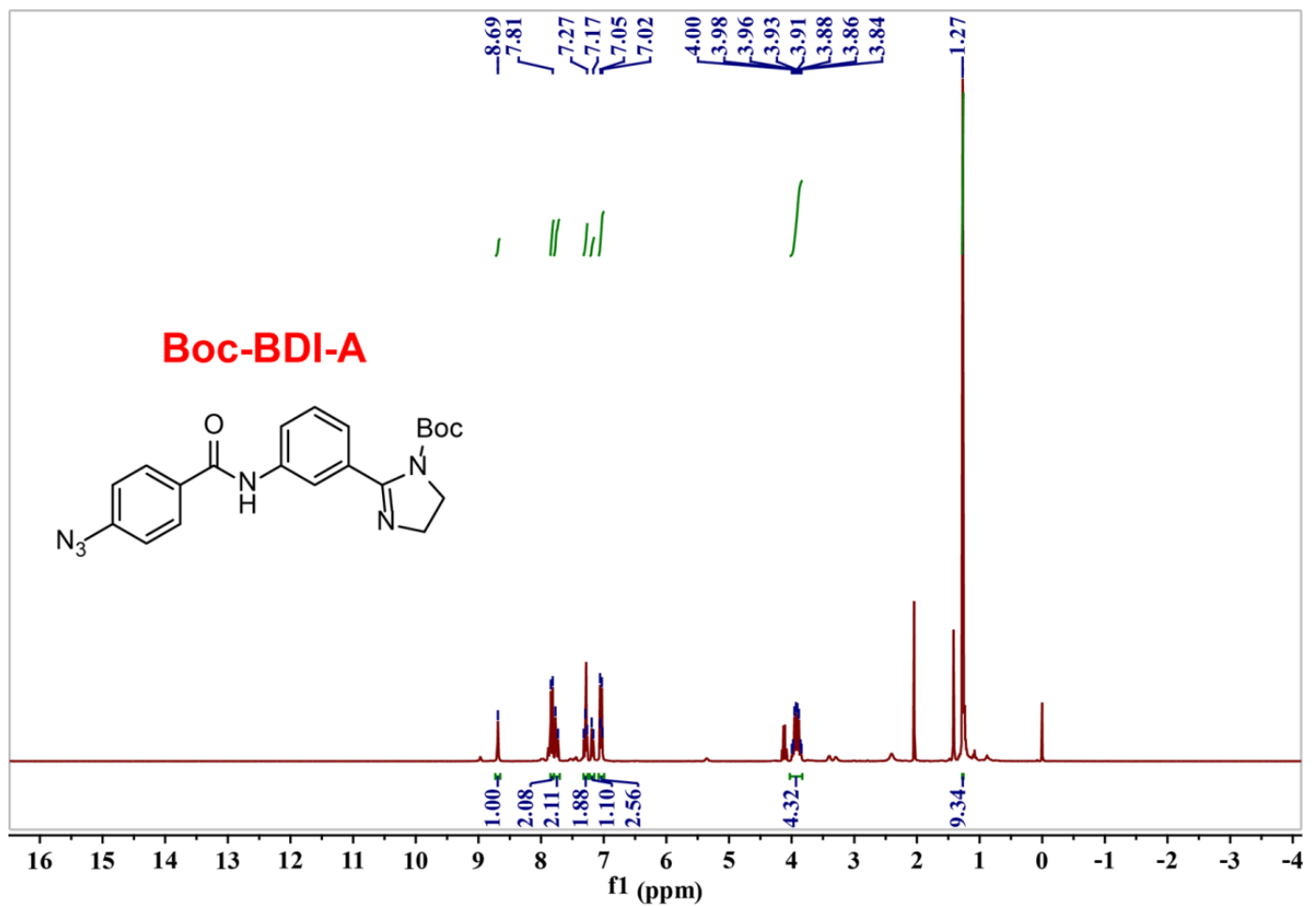

Figure

S26. The

${ }^{1} \mathrm{H}$

NMR

spectrum

of

$\mathrm{N}$-boc-2-(3-(4-azidobenzamido)phenyl)-4,5-dihydro- $1 \mathrm{H}$-imidazole (Boc-BDI-A) in $\mathrm{CDCl}_{3}$. 


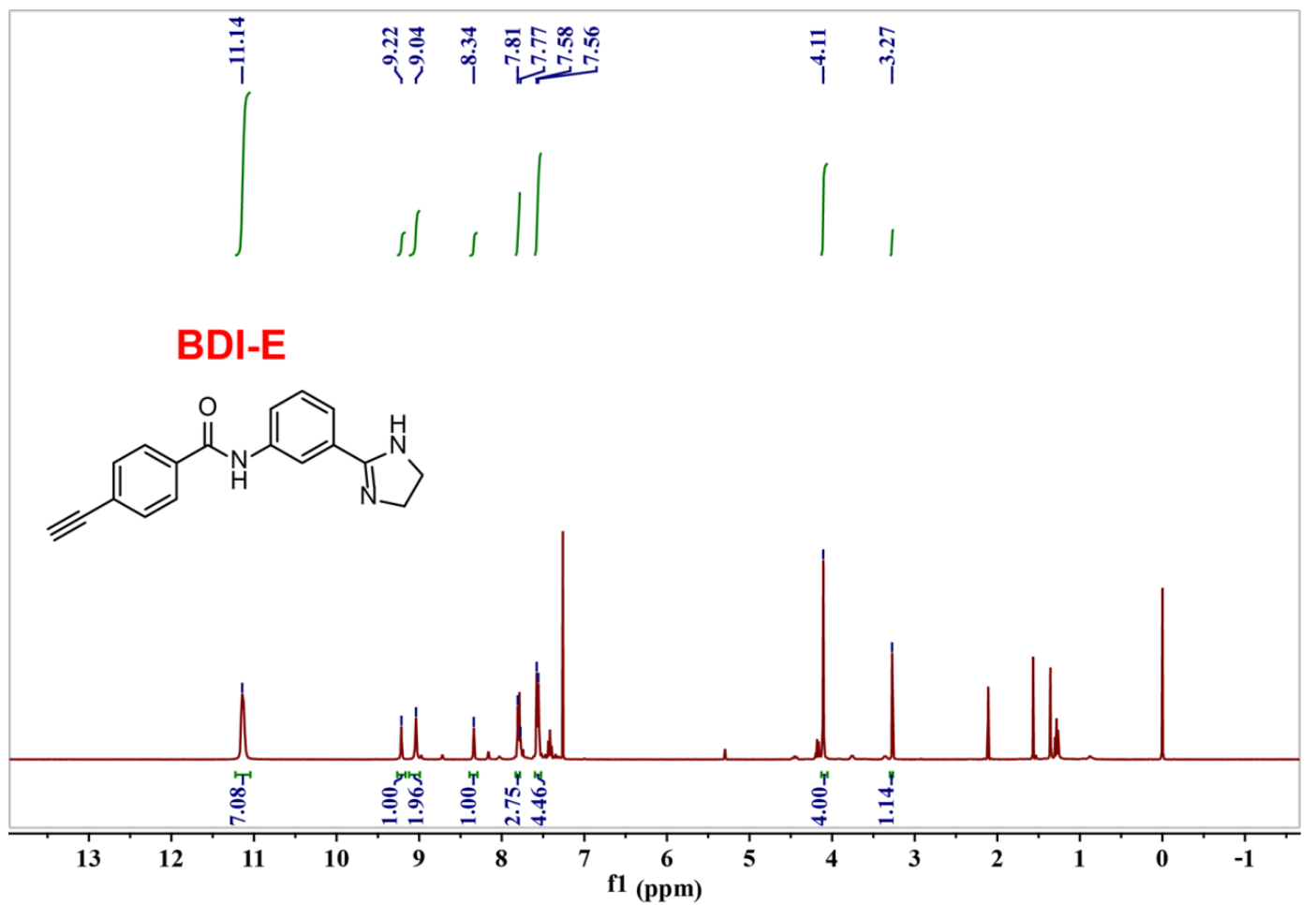

$\begin{array}{llllll}\text { Figure } & \text { S27. } & \text { The } & { }^{1} \mathrm{H} & \text { NMR } & \text { spectrum }\end{array}$

(3-(4-ethynylbenzamido)phenyl)-4,5-dihydro-1 $\mathrm{H}$-imidazole (BDI-E) in $\mathrm{CDCl}_{3}$.

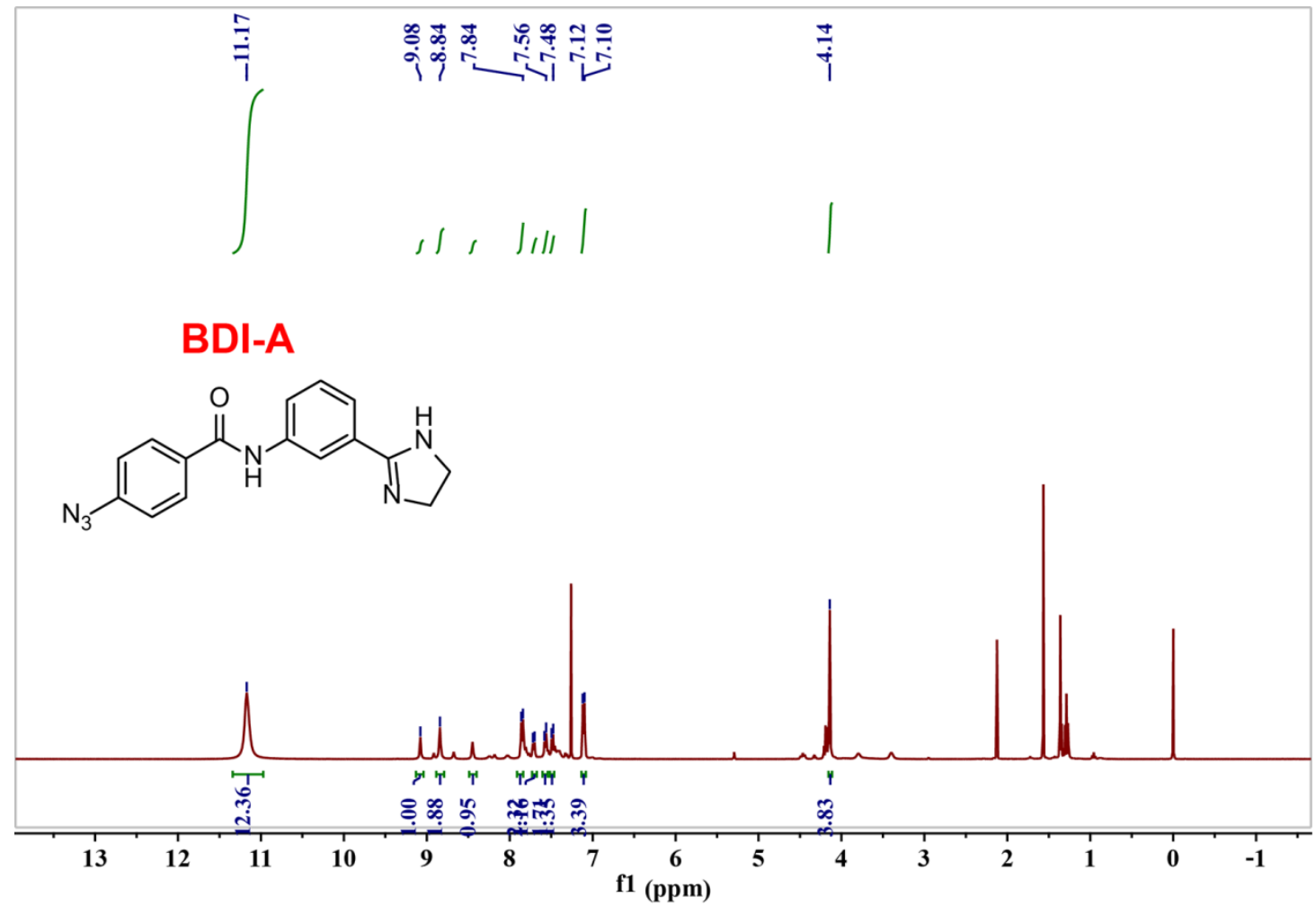

$\begin{array}{lllllll}\text { Figure } & \text { S28. } & \text { The } & { }^{1} \mathrm{H} & \text { NMR } & \text { spectrum } & \text { of }\end{array}$ (3-(4-azidobenzamido)phenyl)-4,5-dihydro-1 $H$-imidazole (BDI-A) in $\mathrm{CDCl}_{3}$. 


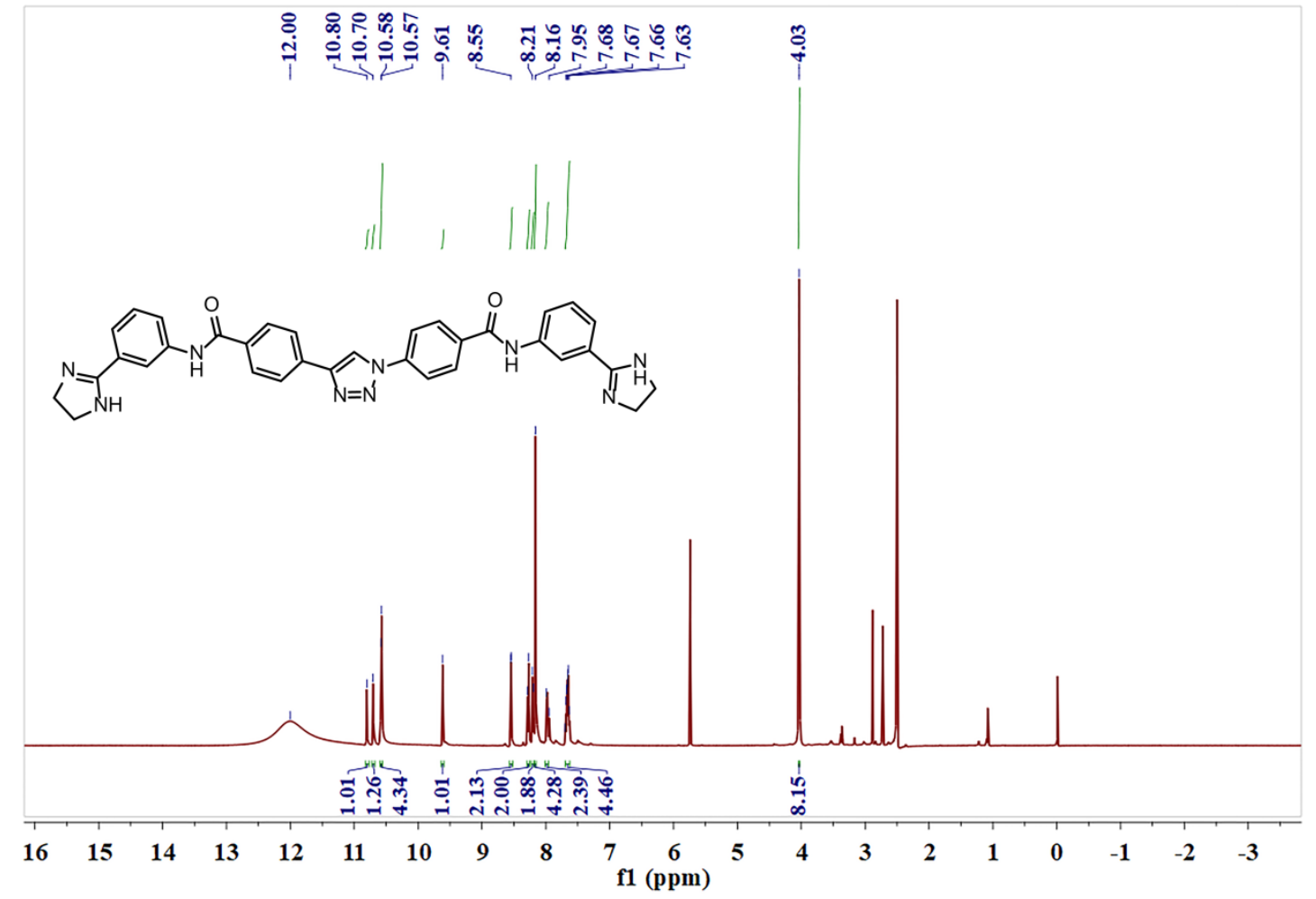

Figure S29. The ${ }^{1} \mathrm{H}$ NMR spectrum of compound 6 in DMSO- $d 6$. 


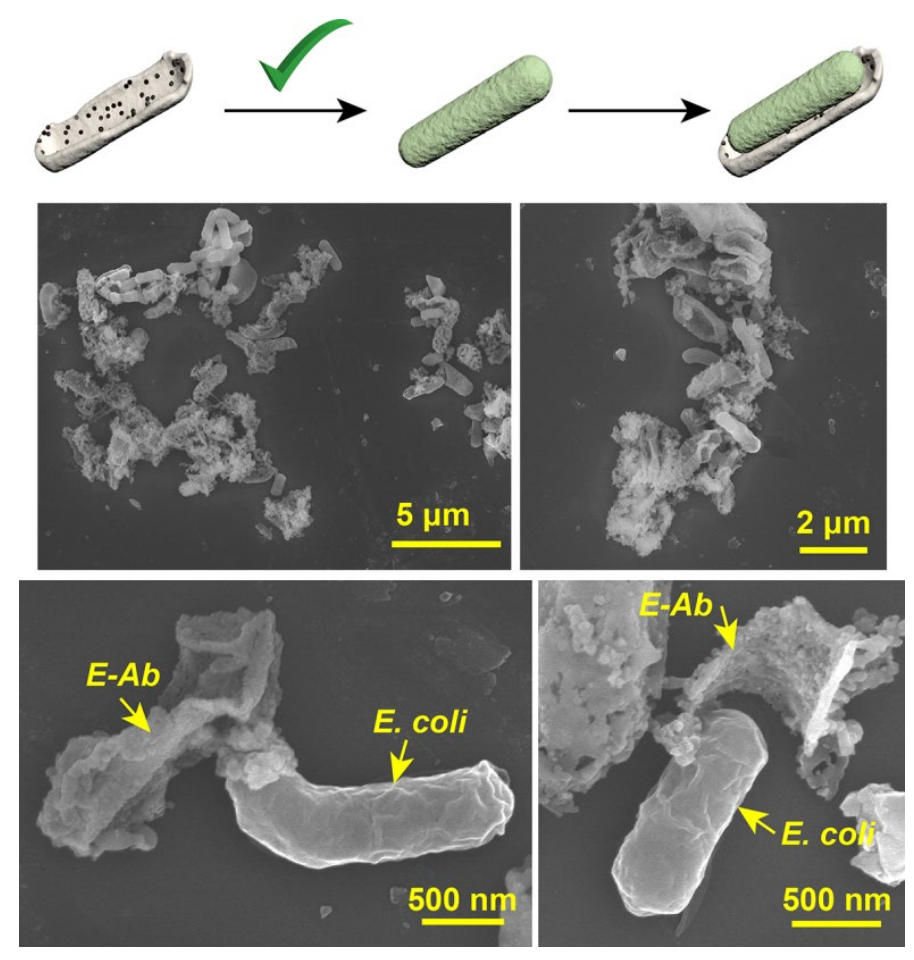

Figure S30. SEM images of $\boldsymbol{E}-\boldsymbol{A} \boldsymbol{b}$ recognizing $E$. coli.

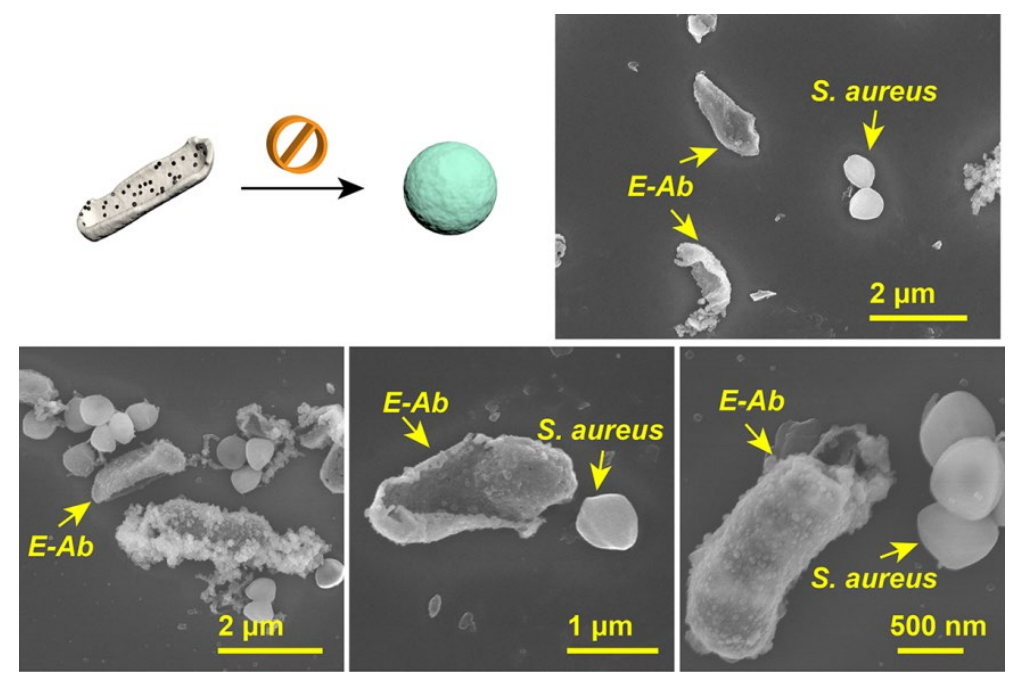

Figure S31. SEM images of $\boldsymbol{E}-\boldsymbol{A} \boldsymbol{b}$ not recognizing $S$. aureus. 

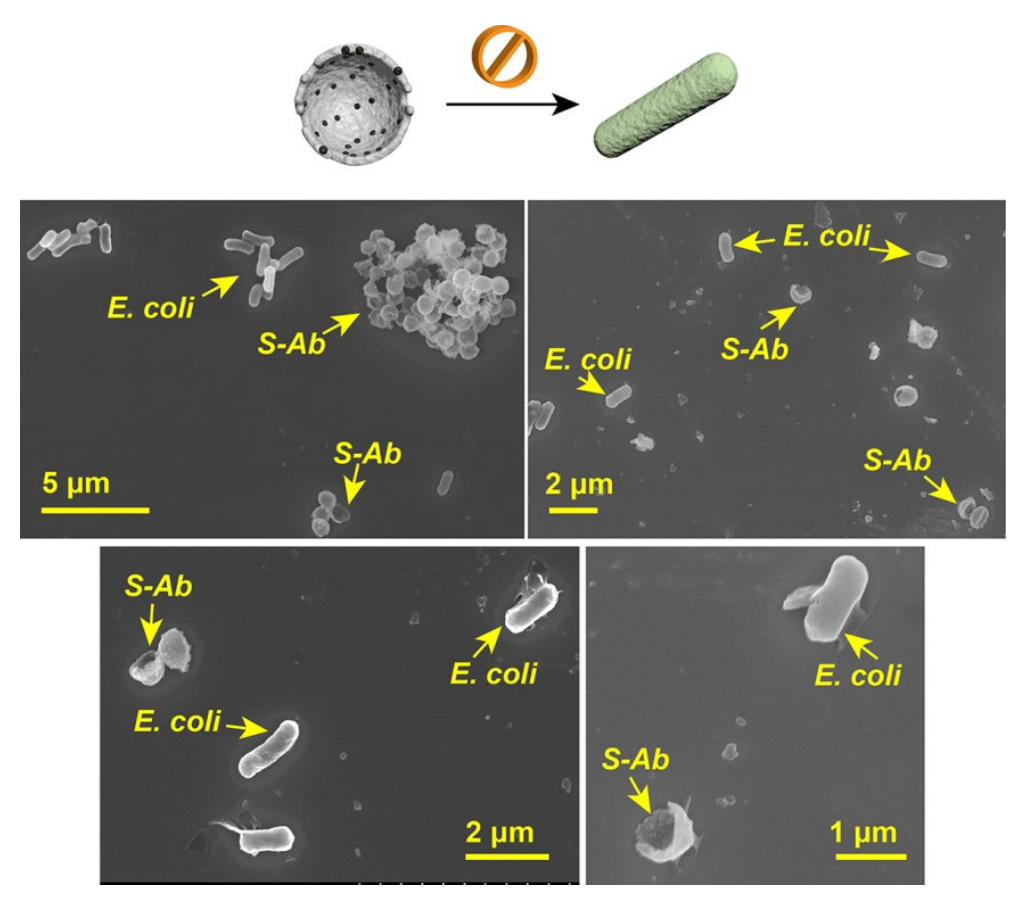

Figure S32. SEM images of $\boldsymbol{S}-\boldsymbol{A} \boldsymbol{b}$ not recognizing $E$. coli.

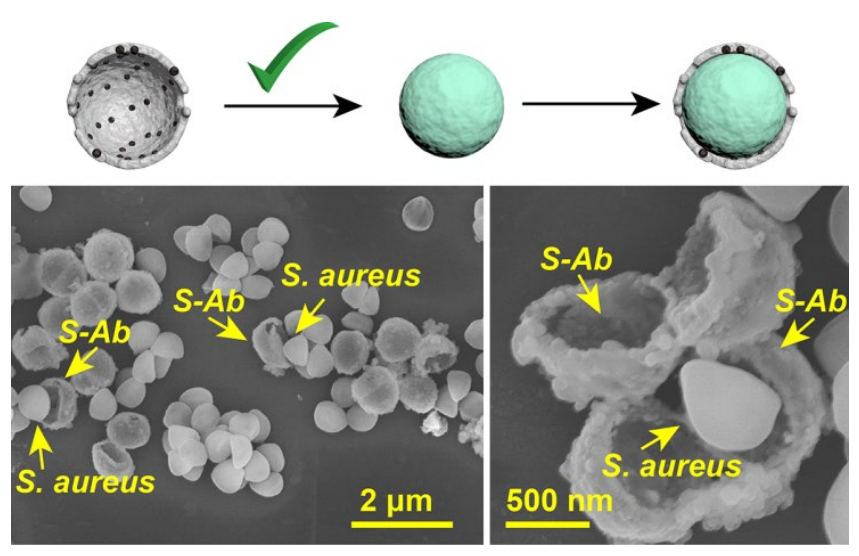

Figure S33. SEM images of $\boldsymbol{S}-\boldsymbol{A} \boldsymbol{b}$ recognizing $S$. aureus. 

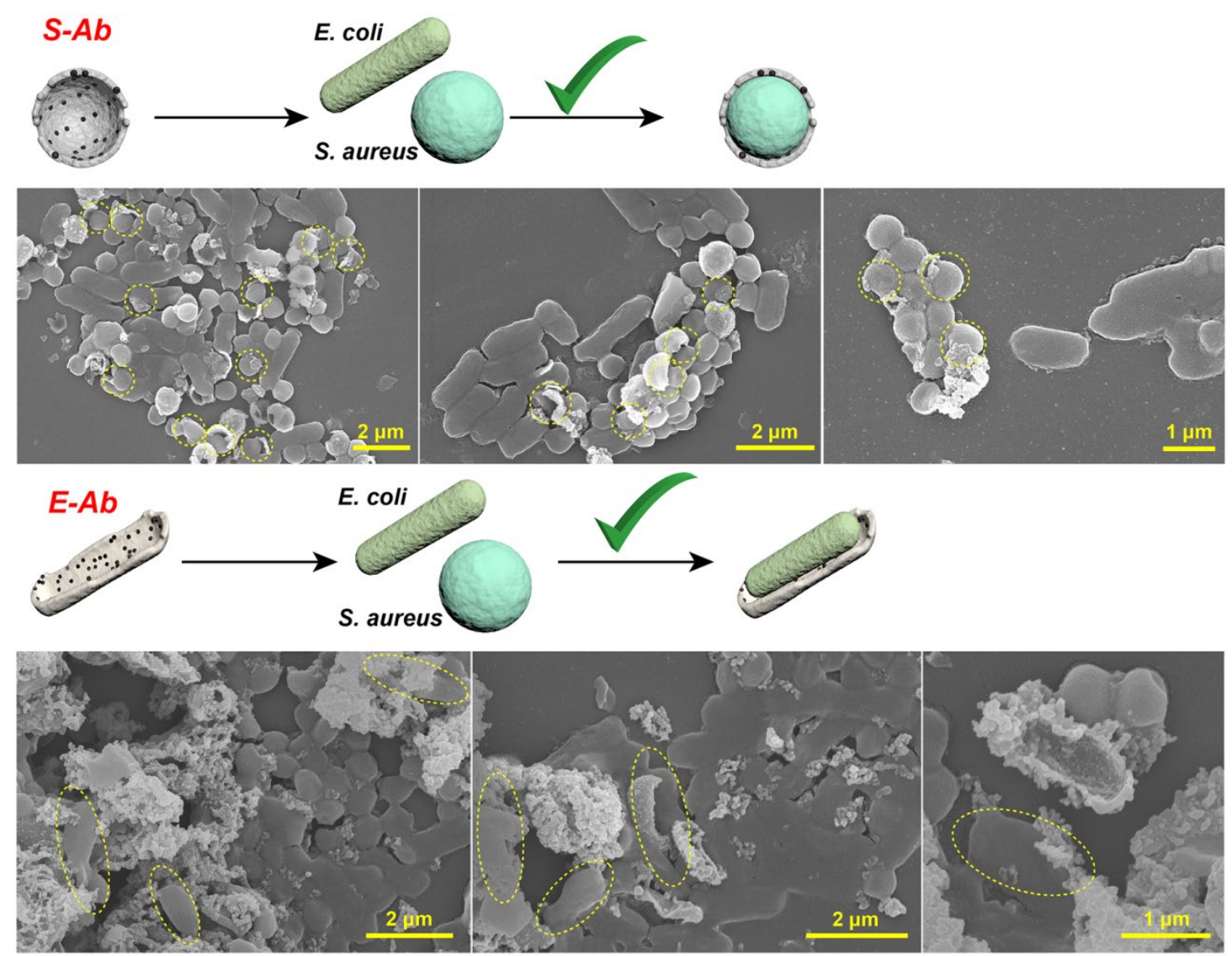

Figure S34. The SEM images confirmed the selective bacteria recognized by $\boldsymbol{E} / \boldsymbol{S}-\boldsymbol{A} \boldsymbol{b}$ in a mixture of E.coli and $S$. aureus. The yellow dotted circles marked the binding of $\boldsymbol{S}-\boldsymbol{A} \boldsymbol{b}$ to the $S$. aureus. And the yellow dotted ellipses marked the binding $\boldsymbol{E}-\boldsymbol{A} \boldsymbol{b}$ of to the E.coli. The results indicated $\boldsymbol{S}-\boldsymbol{A} \boldsymbol{b}$ showed preference to target $S$. aureus instead of $E$. coli, and $\boldsymbol{E}-\boldsymbol{A} \boldsymbol{b}$ showed a preferential targeting of $E$. coli over $S$. aureus. 


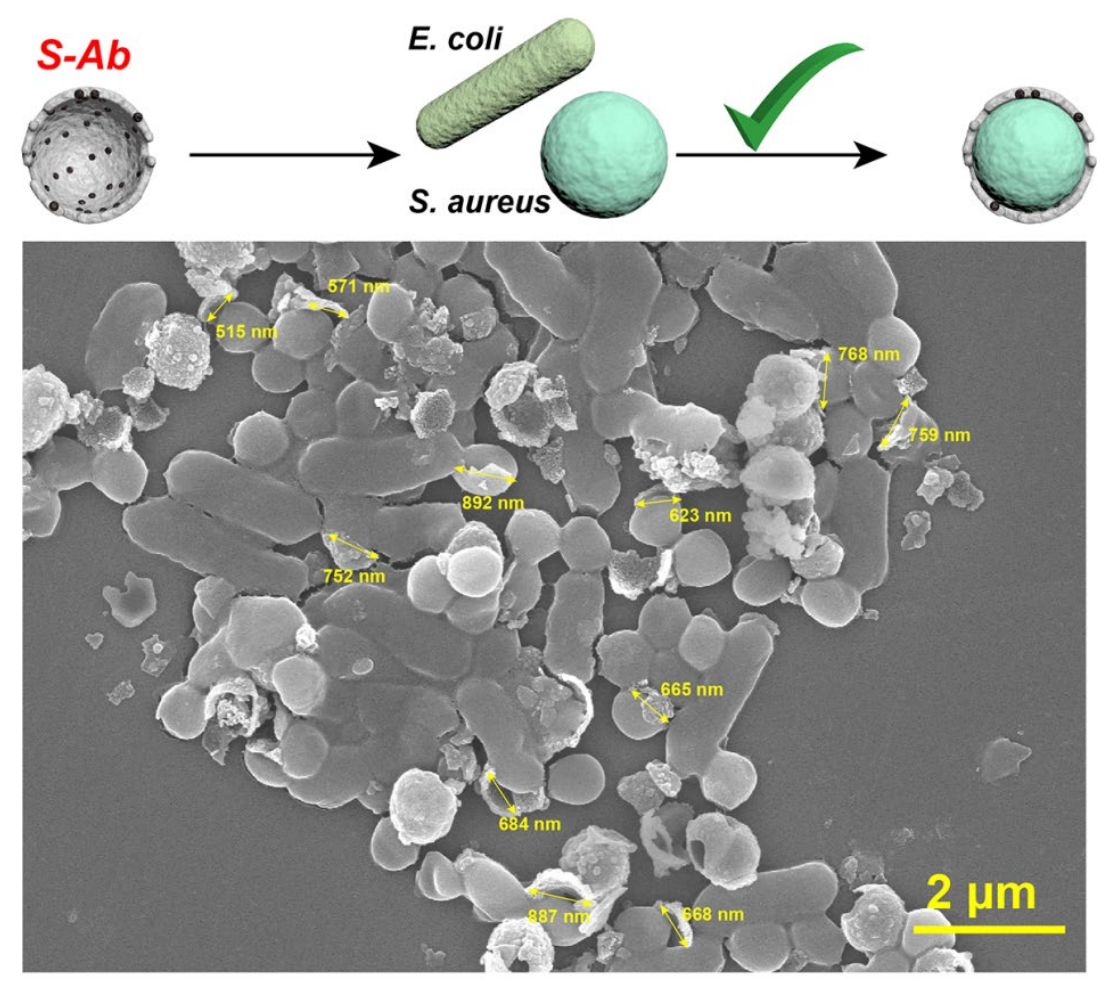

Figure S35. The size of $\boldsymbol{S}$ - $\boldsymbol{A} \boldsymbol{b}$ that could recognize $S$. aureus measured by SEM (Taken from Figure S34). 


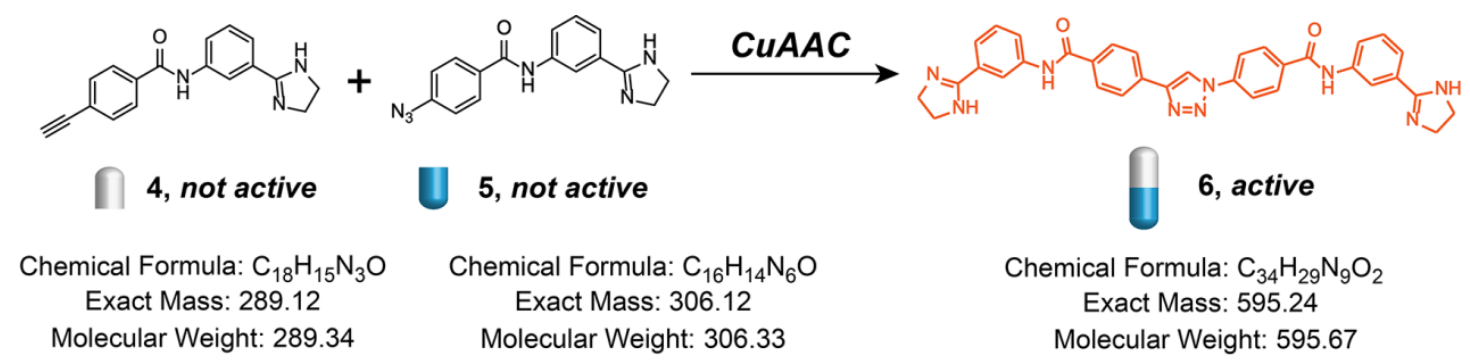

Figure S36. Compounds $\mathbf{4}$ and $\mathbf{5}$ react to generate active antibacterial molecule $\mathbf{6}$ through click reactions, and the structure and molecular weight information of these compounds was analyzed by Chemdraw.

Liquid chromatography-mass spectrometry analysis of the formation of compound 6 .

For LC-MS analysis, we prepared five groups of samples; precursor molecule 4; precursor molecule $\mathbf{5} ; \mathbf{4}+\mathbf{5}+\mathrm{Cu}^{2+}+\mathrm{NaAsc} ; \mathbf{4}+\mathbf{5}+\boldsymbol{E}-\boldsymbol{A} \boldsymbol{b} ; \mathbf{4}+\mathbf{5}+\boldsymbol{S}$ - $\boldsymbol{A} \boldsymbol{b}$. The concentrations of $\mathbf{4}$ and $\mathbf{5}$ were $100 \mu \mathrm{g} / \mathrm{mL}, \mathrm{Cu}^{2+}$ concentration was $100 \mu \mathrm{M}, \mathrm{NaAsc}$ concentration was $500 \mu \mathrm{M}, \boldsymbol{E}-\boldsymbol{A} \boldsymbol{b}$ and $\boldsymbol{S}$ - $\boldsymbol{A} \boldsymbol{b}$ concentration were about $200 \mu \mathrm{g} / \mathrm{mL}$. All samples were carried out in a mix of LB medium and DMF (4:1) analysis. DMF was used to increase the solubility of $\mathbf{4}, \mathbf{5}$ and product 6. 


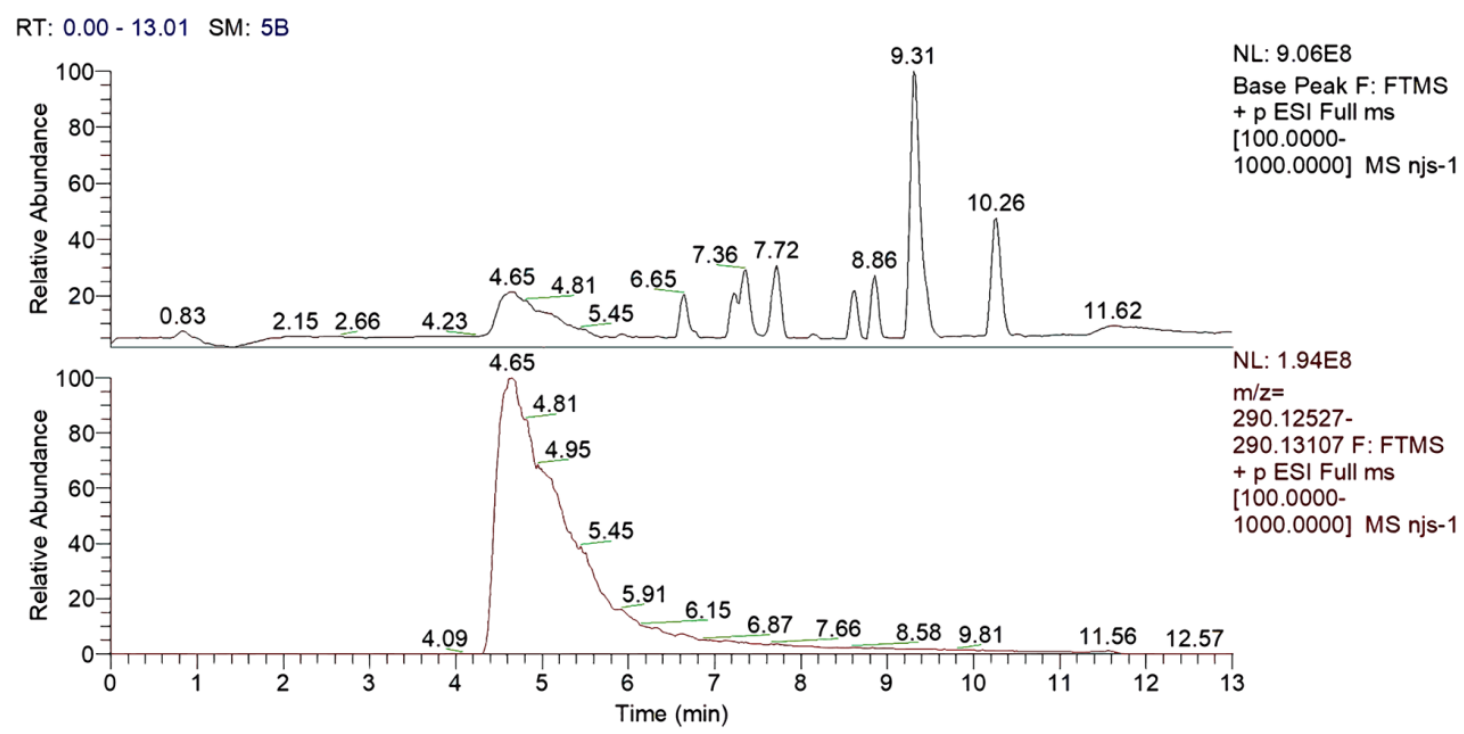

njs-1 \#474-484 RT: 4.85-4.93 AV: 5 SB: 15 3.68-3.85, 5.82-5.93 NL: $1.23 E 8$ T: FTMS + p ESI Full ms [100.0000-1000.0000]

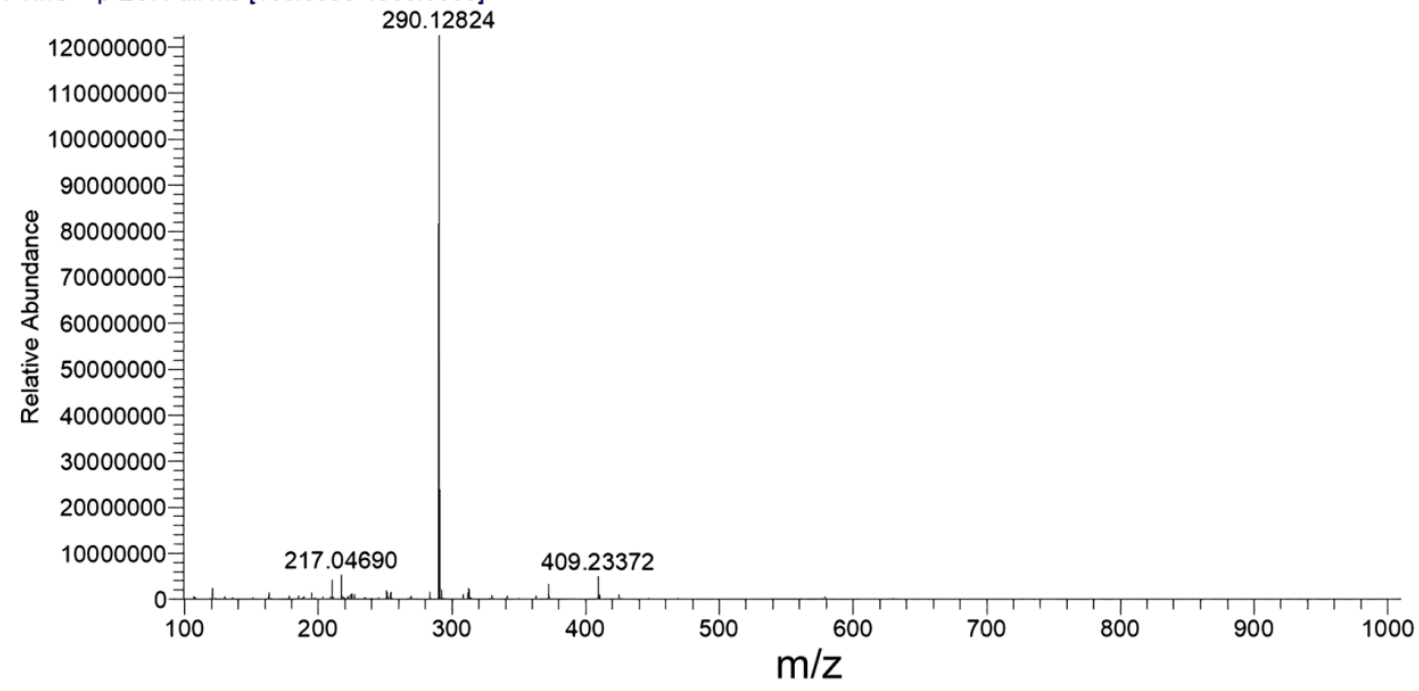

Figure S37. LC-MS analysis of inactive molecule 4 in the mixed solution of LB medium and $\operatorname{DMF}(4: 1)$. 
RT: $0.00-13.00$ SM: $5 B$
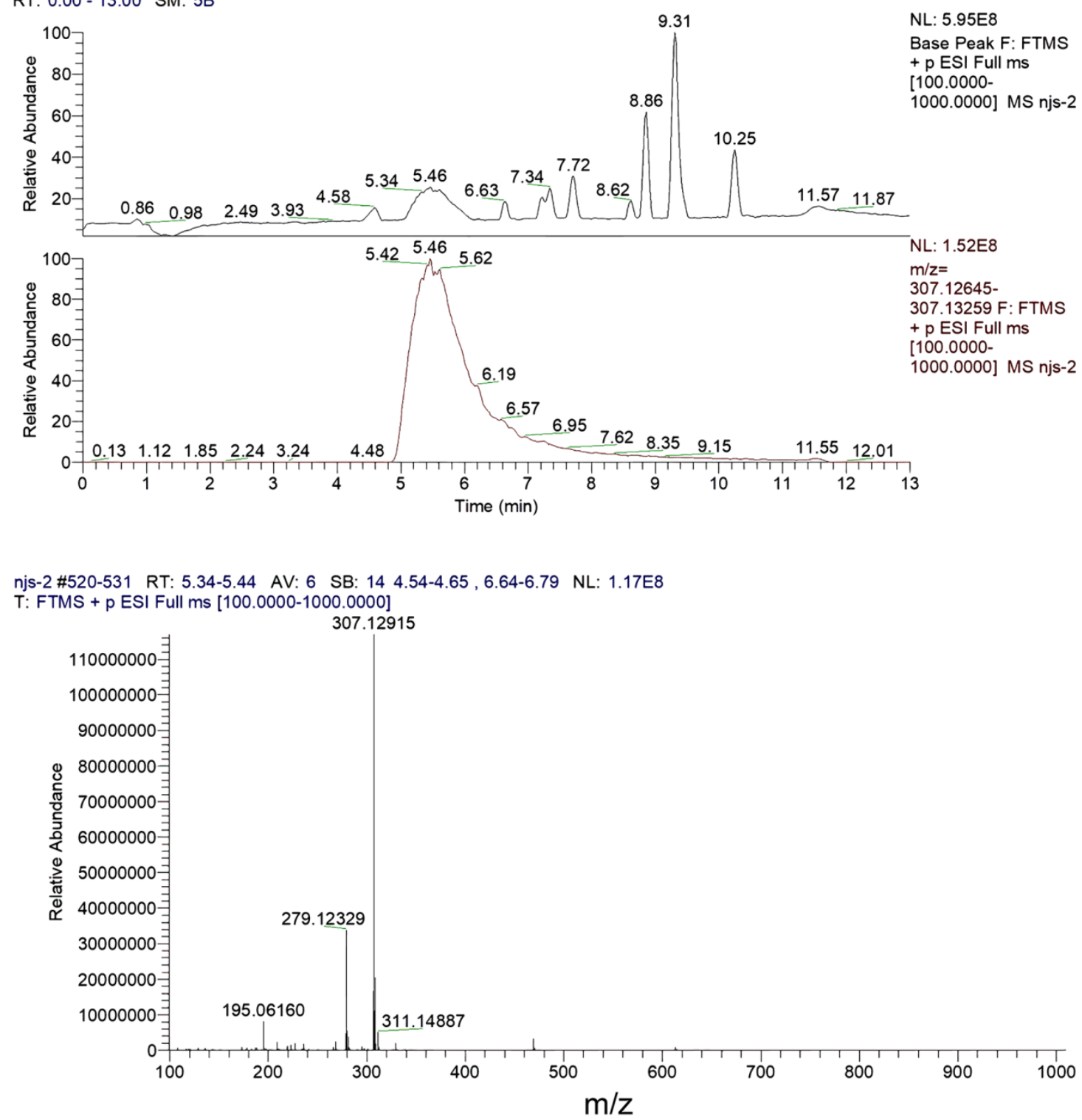

Figure S38. LC-MS analysis of inactive molecule $\mathbf{5}$ in the mixed solution of LB medium and $\operatorname{DMF}(4: 1)$ 


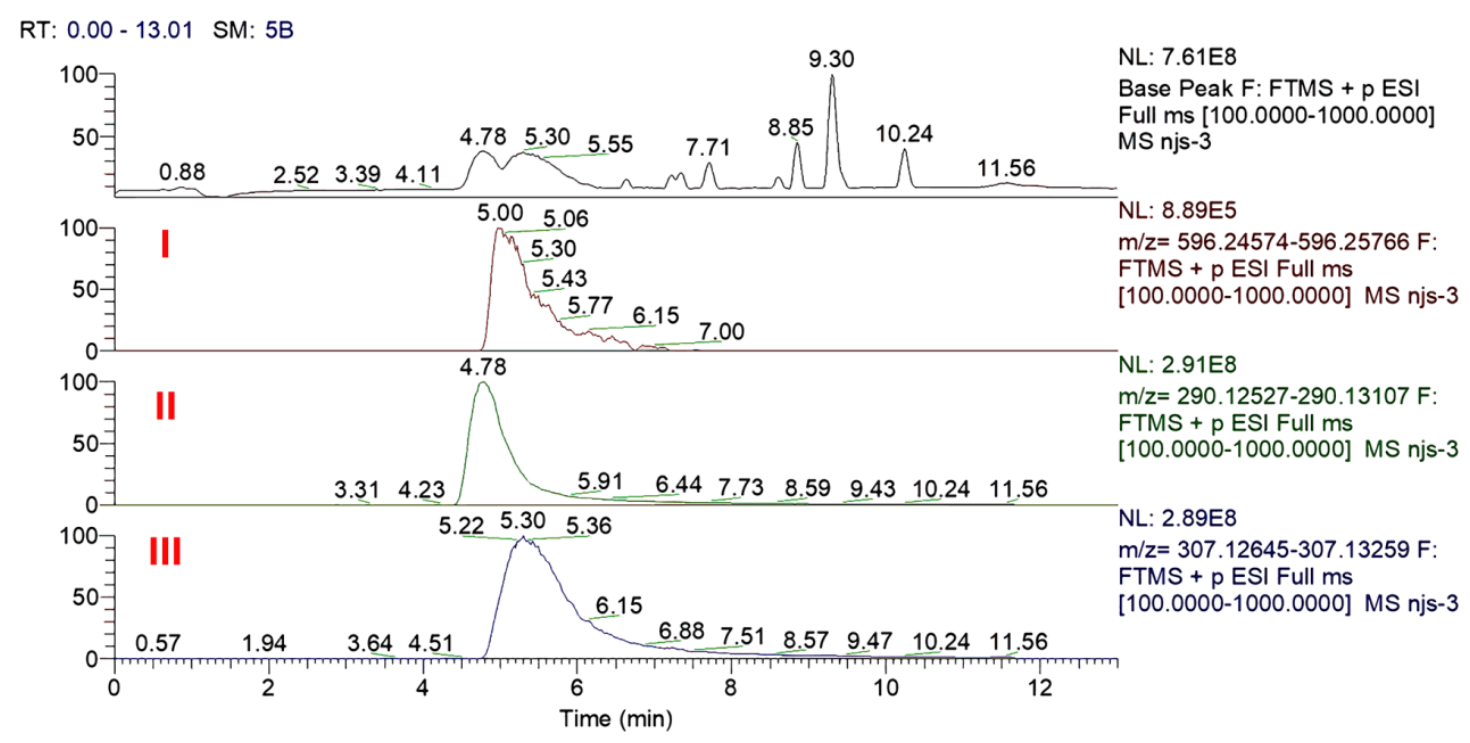

Figure S39a. LC-MS analysis of inactive molecules $\mathbf{4}$ and $\mathbf{5}$ in the mixed solution of LB medium and DMF (4:1) in the presence of copper and sodium ascorbate to generate active molecule 6. 


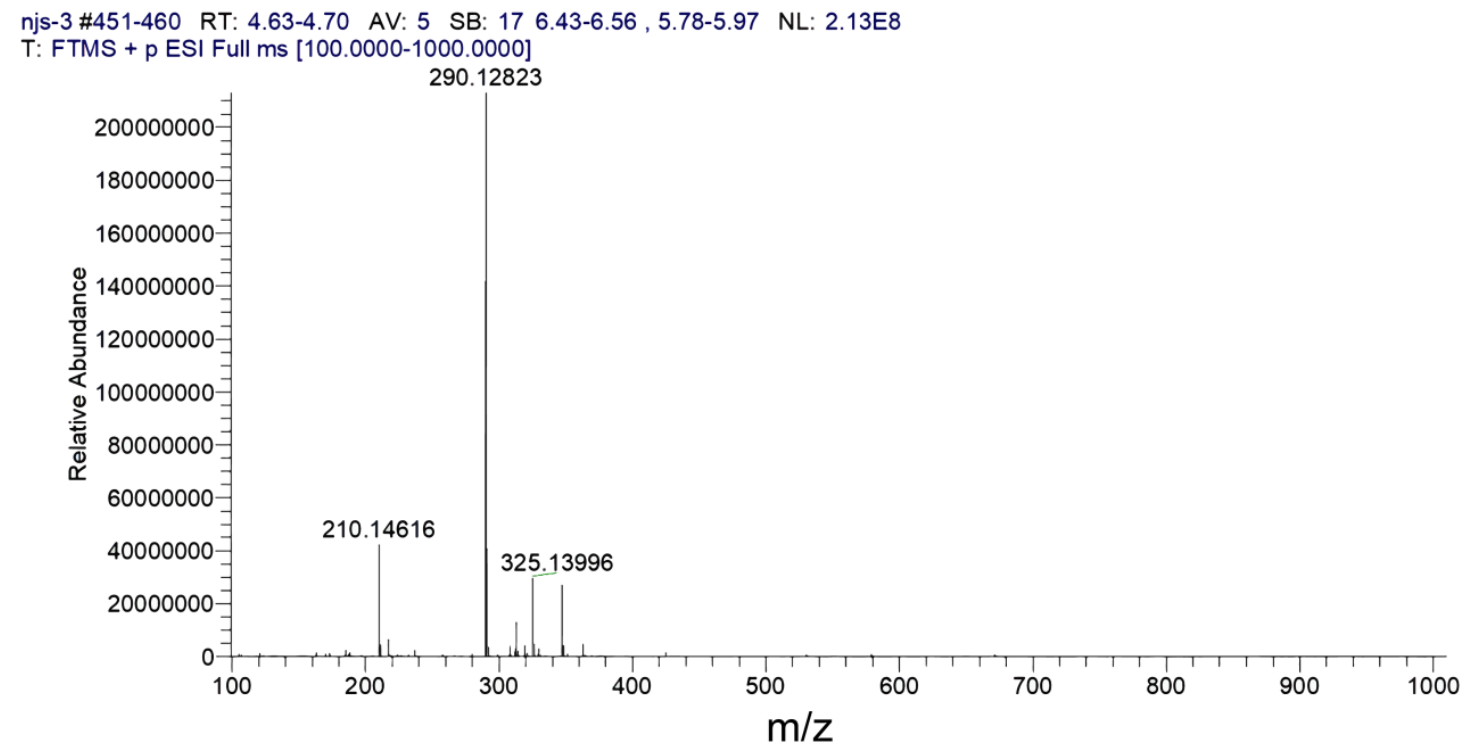

njs-3 \#451-460 RT: $4.63-4.70$ AV: 5 SB: 17 6.43-6.56, 5.78-5.97 NL: $2.13 E 8$

T: FTMS + p ESI Full ms [100.0000-1000.0000]

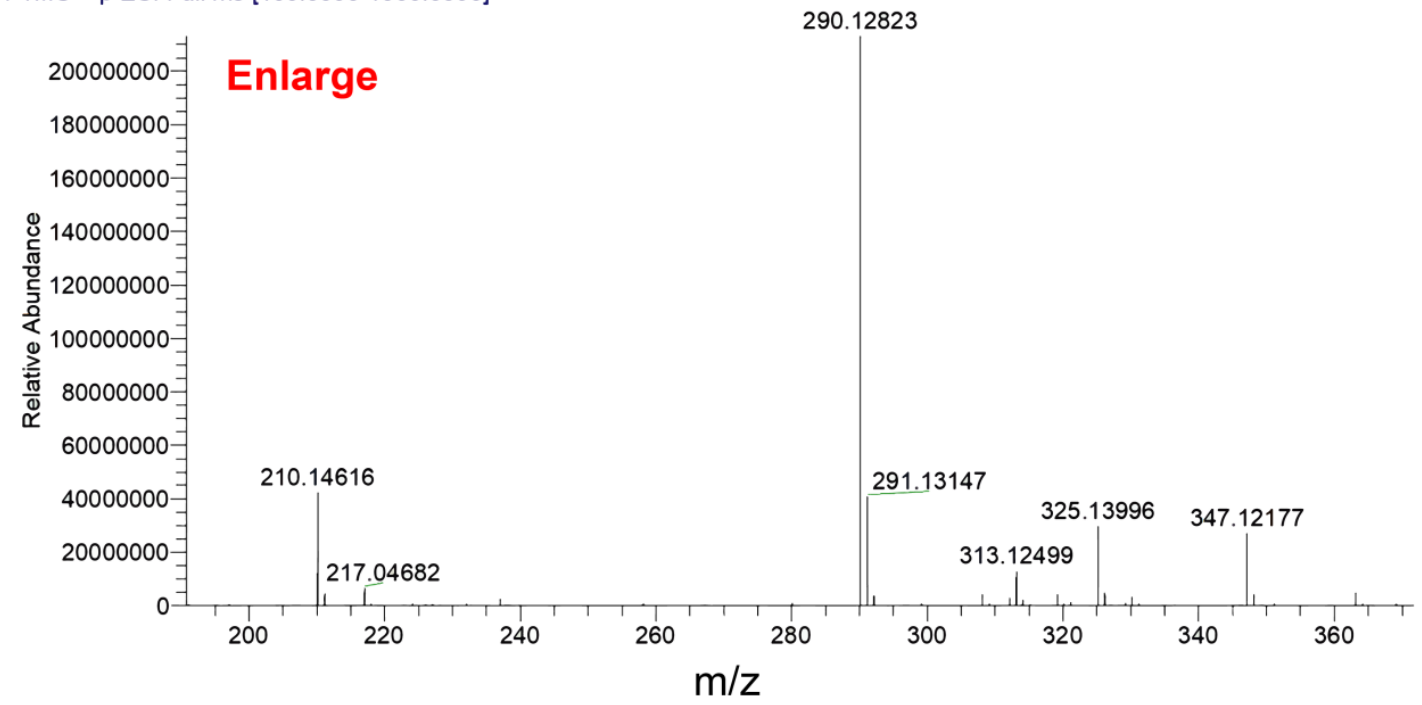

Figure S39b. LC-MS analysis of inactive molecules $\mathbf{4}$ and $\mathbf{5}$ in the mixed solution of LB medium and DMF (4:1) in the presence of copper and sodium ascorbate to generate active molecule 6. The results in this figure indicated the presence of 4 in the mixture. 


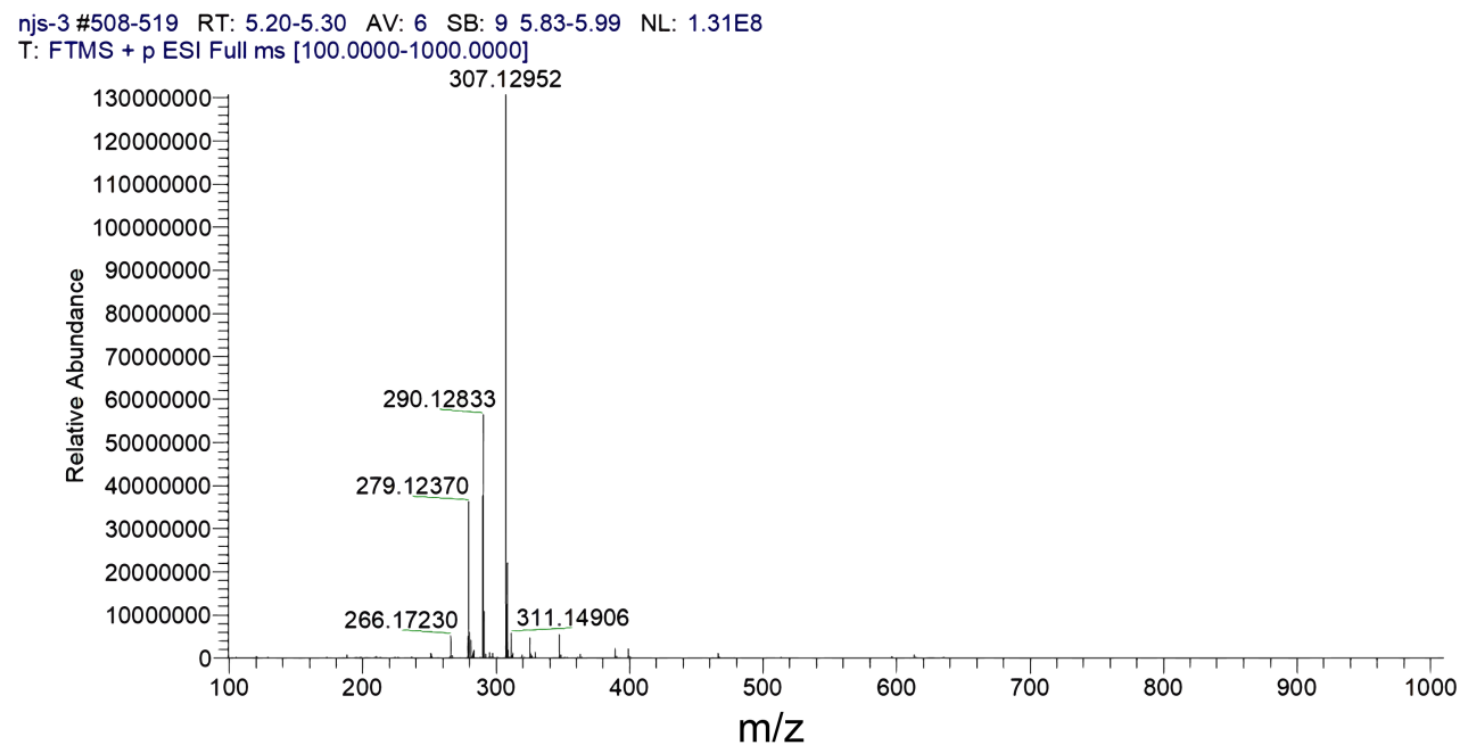

njs-3\#508-519 RT: 5.20-5.30 AV: 6 SB: 9 5.83-5.99 NL: $1.31 \mathrm{E} 8$ T: FTMS + p ESI Full ms [100.0000-1000.0000]

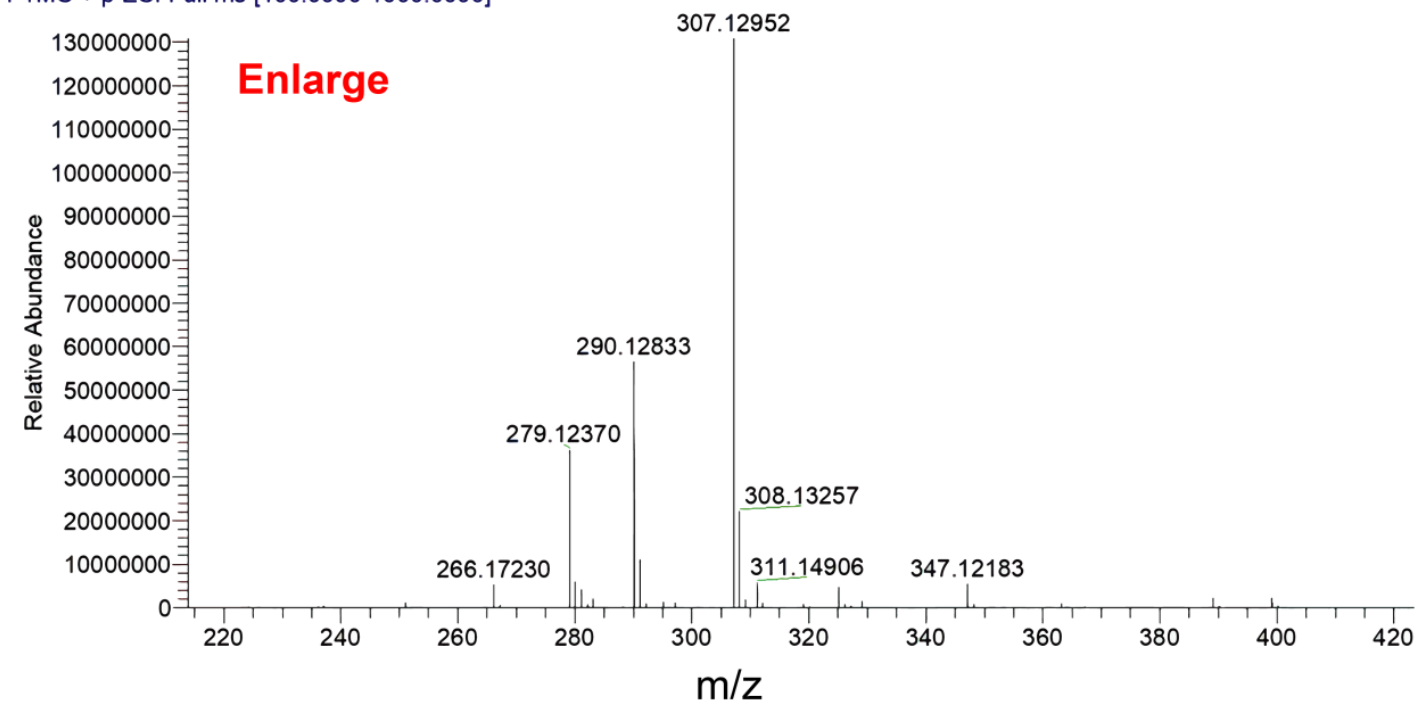

Figure S39c. LC-MS analysis of inactive molecules $\mathbf{4}$ and $\mathbf{5}$ in the mixed solution of LB medium and DMF (4:1) in the presence of copper and sodium ascorbate to generate active molecule $\mathbf{6}$. The results in this figure indicated the presence of $\mathbf{5}$ in the mixture. 

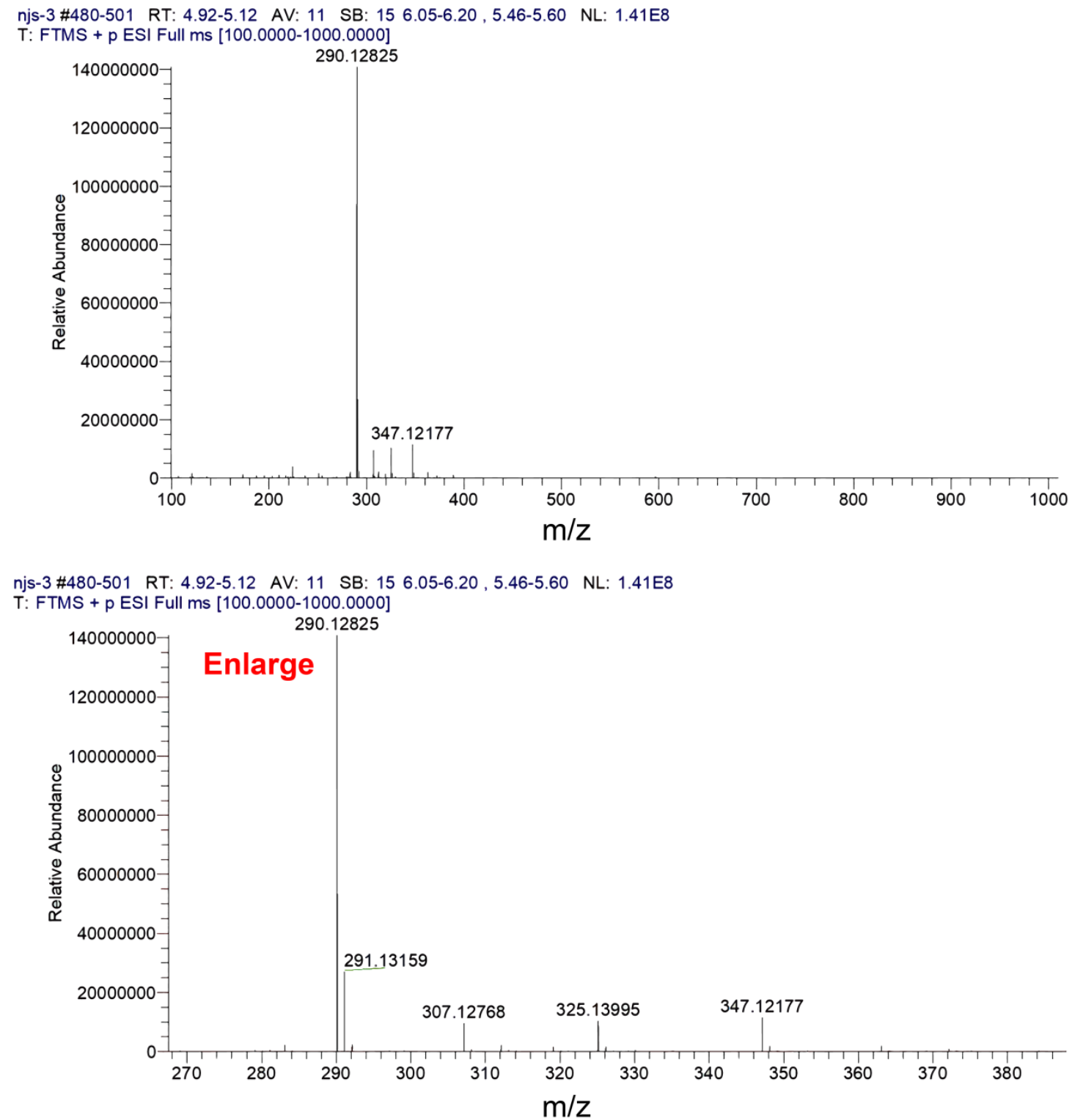

Figure S39d. LC-MS analysis of inactive molecules $\mathbf{4}$ and $\mathbf{5}$ in the mixed solution of LB medium and DMF (4:1) in the presence of copper and sodium ascorbate to generate active molecule 6 . The results in this figure indicated the presence of $\mathbf{4}$ and $\mathbf{5}$ in the mixture. 


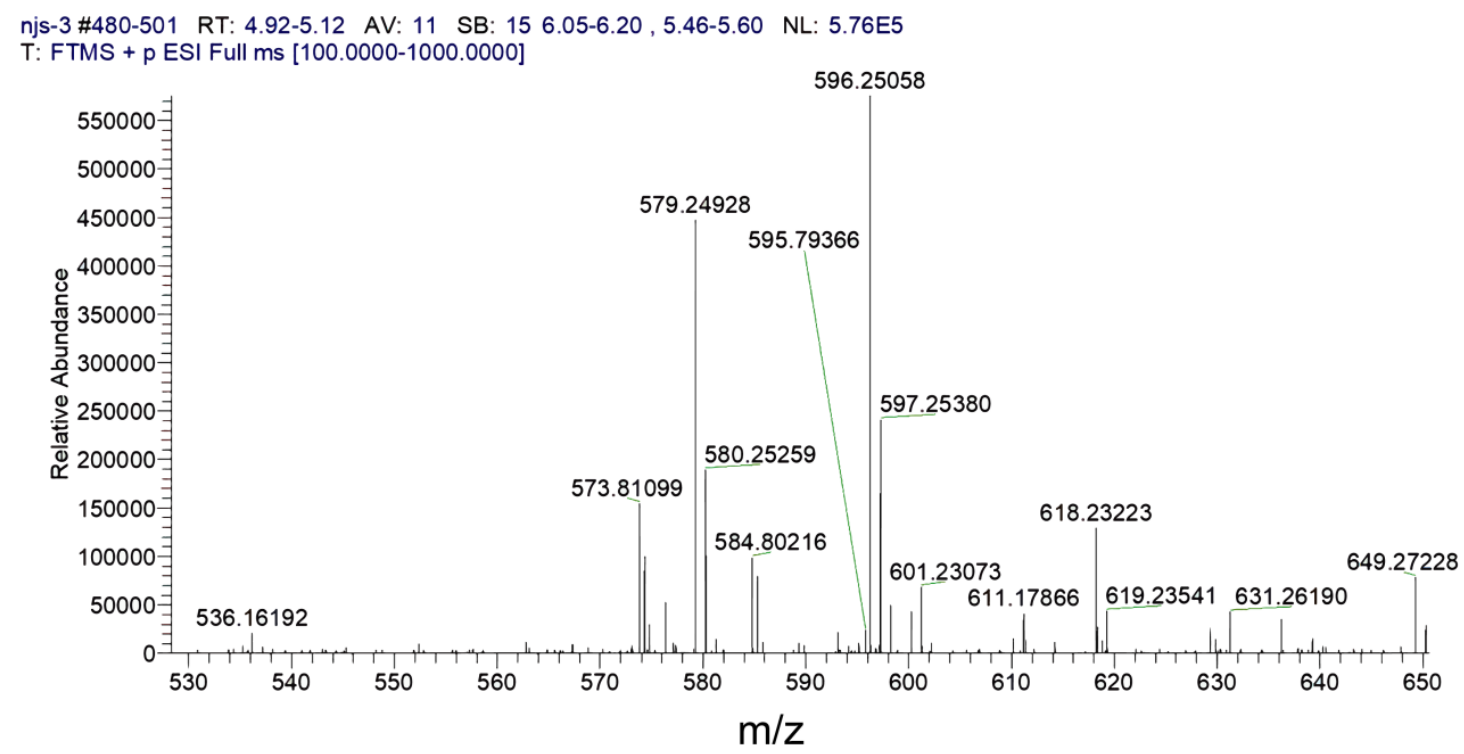

Figure S39e. LC-MS analysis of inactive molecules 4 and $\mathbf{5}$ in the mixed solution of LB medium and DMF (4:1) in the presence of copper and sodium ascorbate to generate active molecule 6. The final product $\mathbf{6}(\mathbf{5 9 6 . 2 5 0 5 8})$ confirmed that the $\mathbf{C u A A C}$ reaction catalyzed by $\mathrm{Cu}+$ and $\mathrm{NaAsc}$ occurred. 


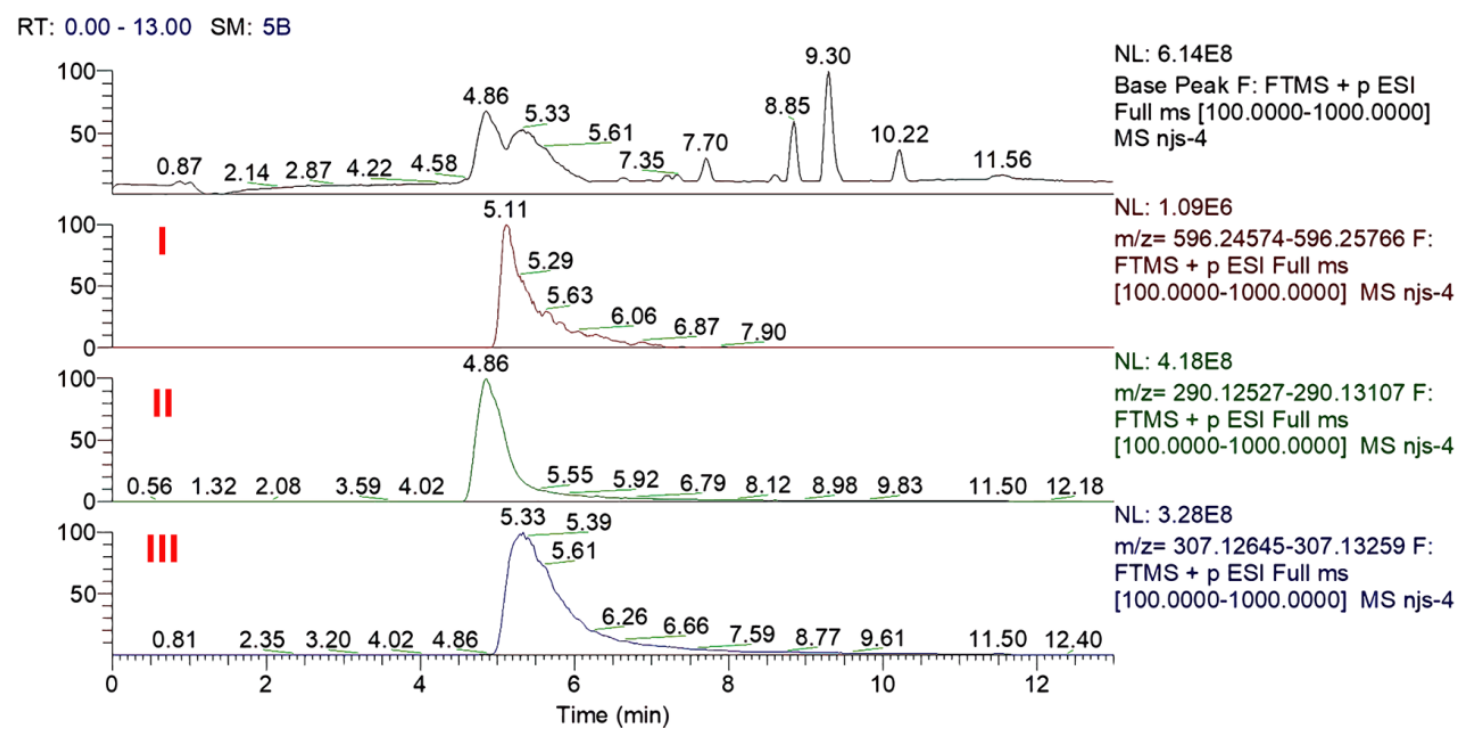

Figure S40a. LC-MS analysis of inactive molecules 4 and 5 in the mixed solution of LB medium and DMF (4:1) in the presence of $\boldsymbol{E}-\boldsymbol{A} \boldsymbol{b}$ to generate active molecule 6. 


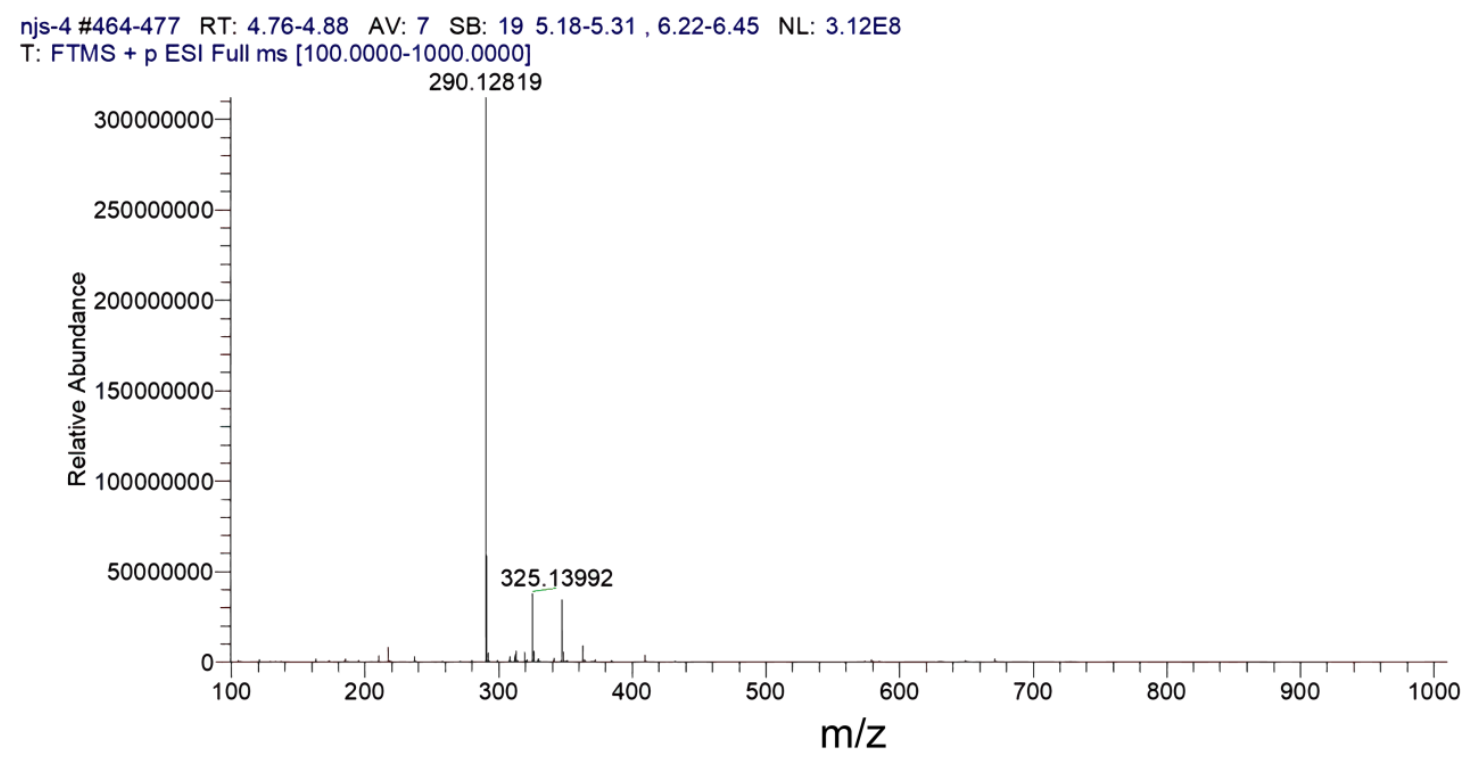

njs-4 \#464-477 RT: 4.76-4.88 AV: 7 SB: 19 5.18-5.31, 6.22-6.45 NL: 3.12E8 T: FTMS + p ESI Full ms [100.0000-1000.0000]

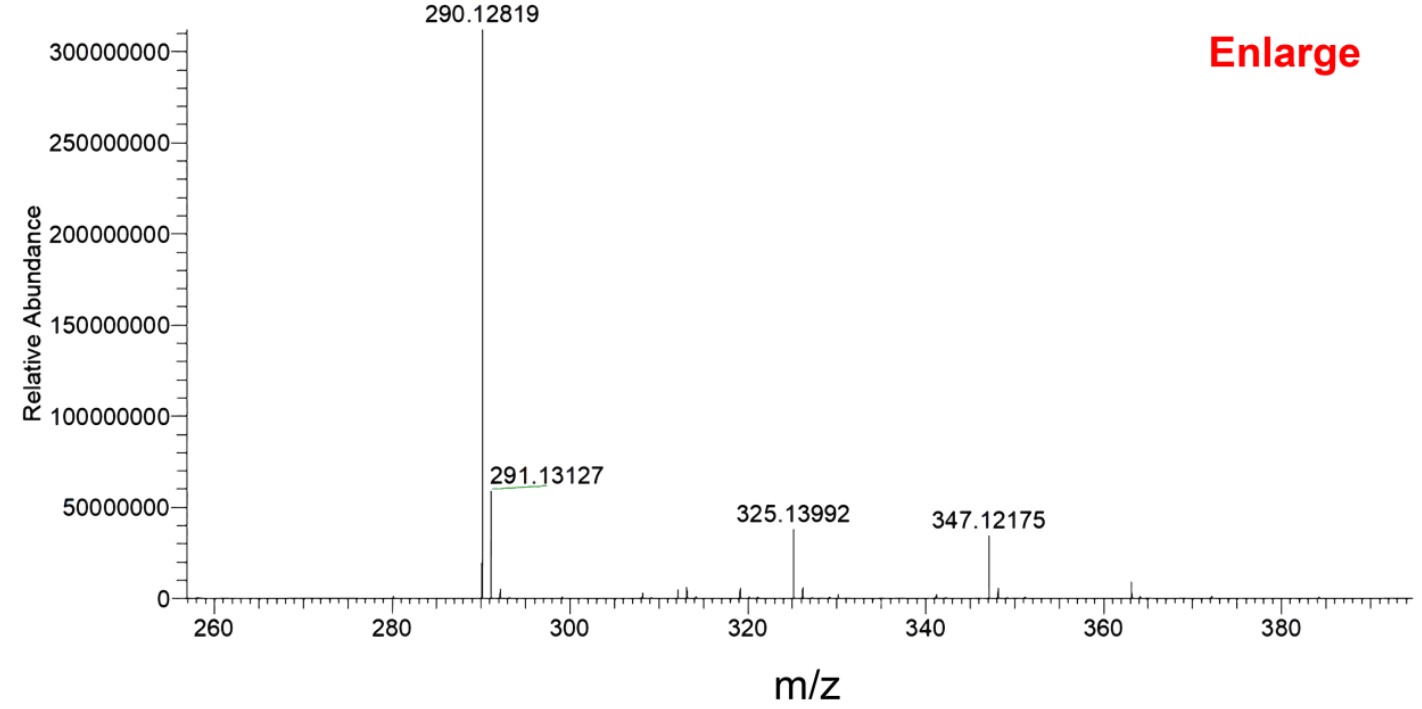

Figure S40b. LC-MS analysis of inactive molecules $\mathbf{4}$ and $\mathbf{5}$ in the mixed solution of LB medium and DMF (4:1) in the presence of $\boldsymbol{E}-\boldsymbol{A} \boldsymbol{b}$ to generate active molecule $\mathbf{6}$. The results in this figure indicated the presence of 4 in the mixture. 


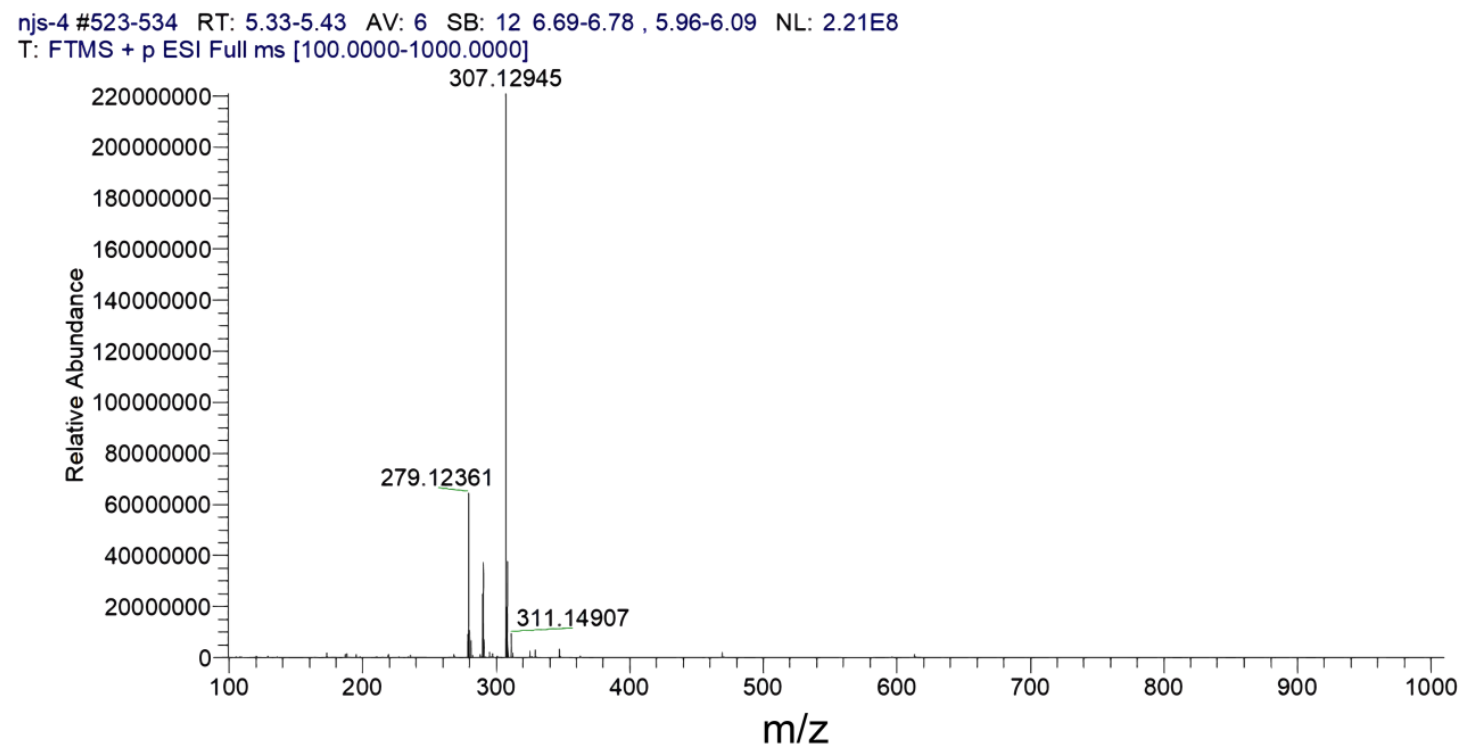

njs-4 \#523-534 RT: 5.33-5.43 AV: 6 SB: 12 6.69-6.78, 5.96-6.09 NL: 2.21E8 T: FTMS + p ESI Full ms [100.0000-1000.0000]

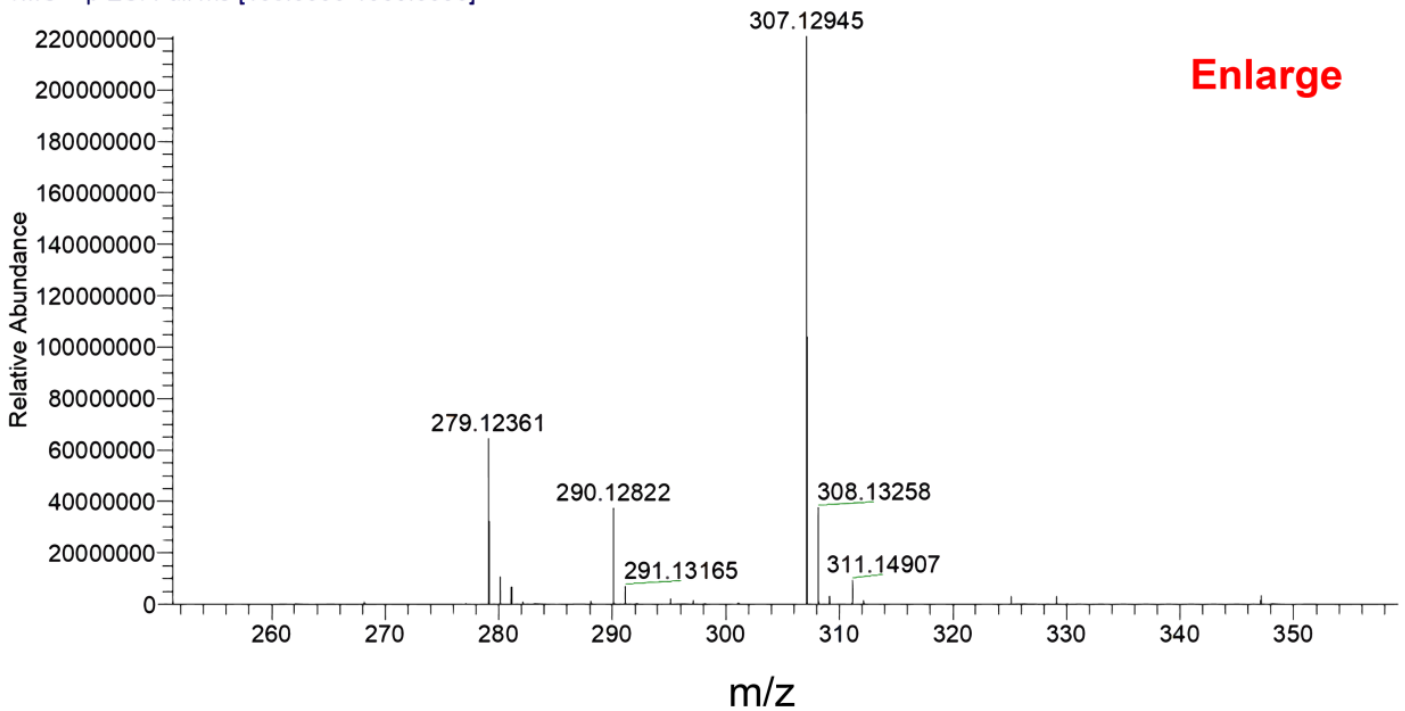

Figure S40c. LC-MS analysis of inactive molecules $\mathbf{4}$ and $\mathbf{5}$ in the mixed solution of LB medium and DMF (4:1) in the presence of $\boldsymbol{E}-\boldsymbol{A} \boldsymbol{b}$ to generate active molecule $\mathbf{6}$. The results in this figure indicated the presence of 5 in the mixture. 


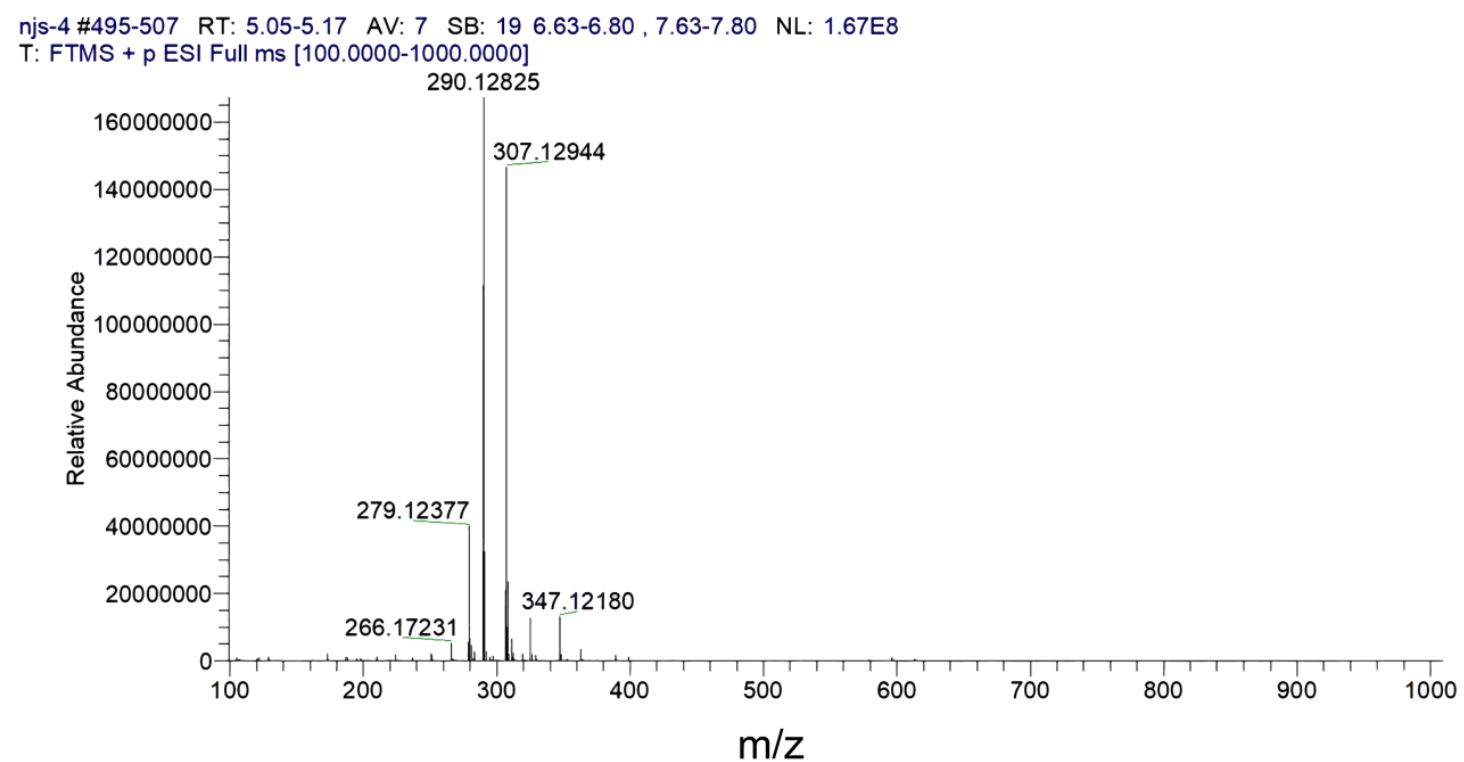

njs-4 \#495-507 RT: 5.05-5.17 AV: 7 SB: $196.63-6.80,7.63-7.80$ NL: $1.67 E 8$ T: FTMS + p ESI Full ms [100.0000-1000.0000]

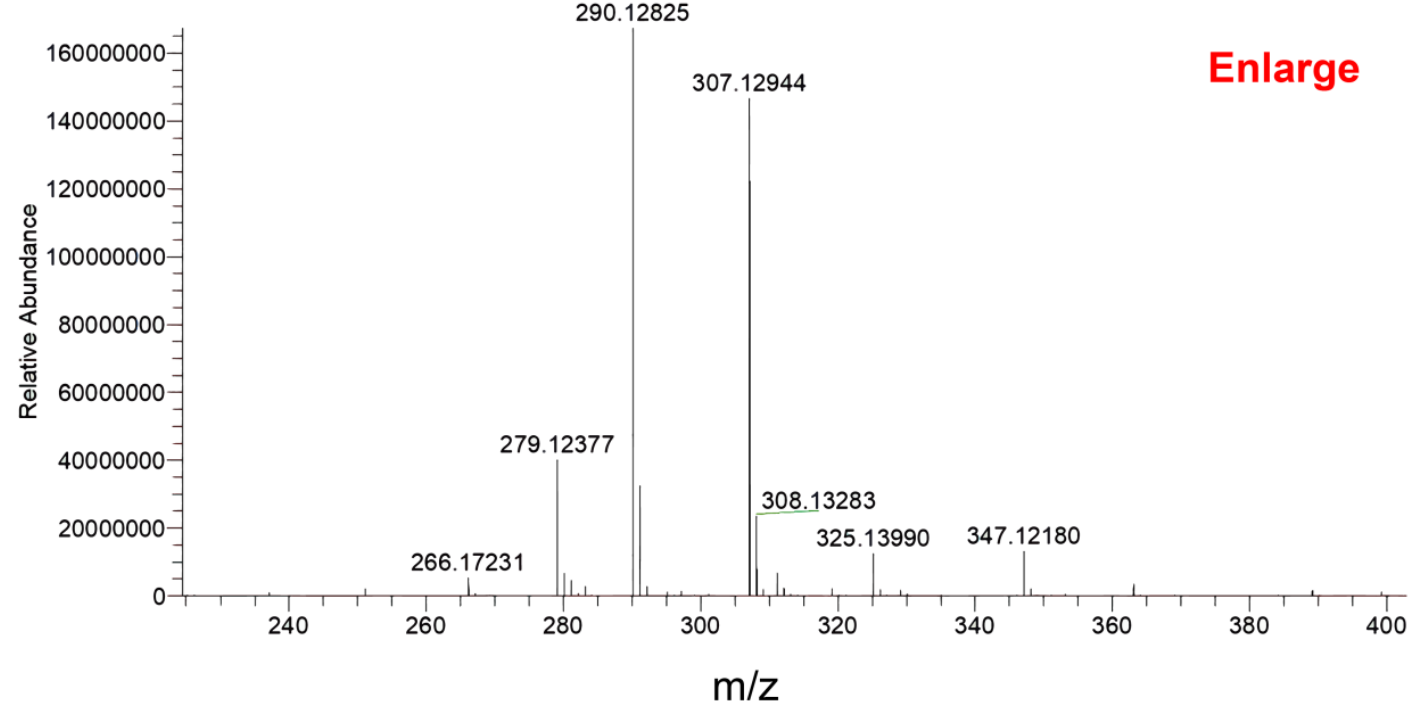

Figure S40d. LC-MS analysis of inactive molecules $\mathbf{4}$ and $\mathbf{5}$ in the mixed solution of LB medium and DMF (4:1) in the presence of $\boldsymbol{E}-\boldsymbol{A} \boldsymbol{b}$ to generate active molecule $\mathbf{6}$. The results in this figure indicated the presence of $\mathbf{4}$ and $\mathbf{5}$ in the mixture. 
njs-4 \#495-507 RT: $5.05-5.17$ AV: 7 SB: 19 6.63-6.80, 7.63-7.80 NL: 9.42E5

T: FTMS + p ESI Full ms [100.0000-1000.0000]

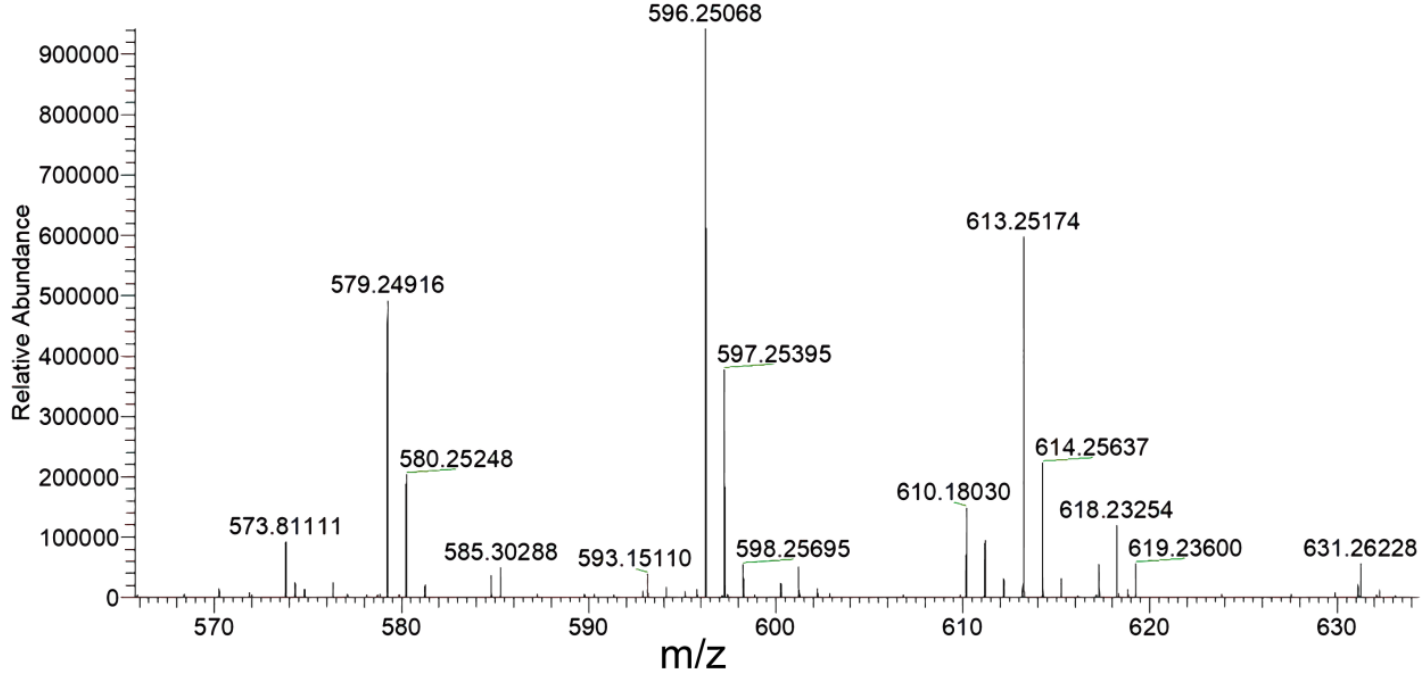

Figure S40e. LC-MS analysis of inactive molecules 4 and $\mathbf{5}$ in the mixed solution of LB

medium and DMF (4:1) in the presence of $\boldsymbol{E}-\boldsymbol{A} \boldsymbol{b}$ to generate active molecule $\mathbf{6}$. The final product 6 (596.25068) confirmed that the $\mathrm{CuAAC}$ reaction catalyzed by $\boldsymbol{E}-\boldsymbol{A b}$ occurred. 


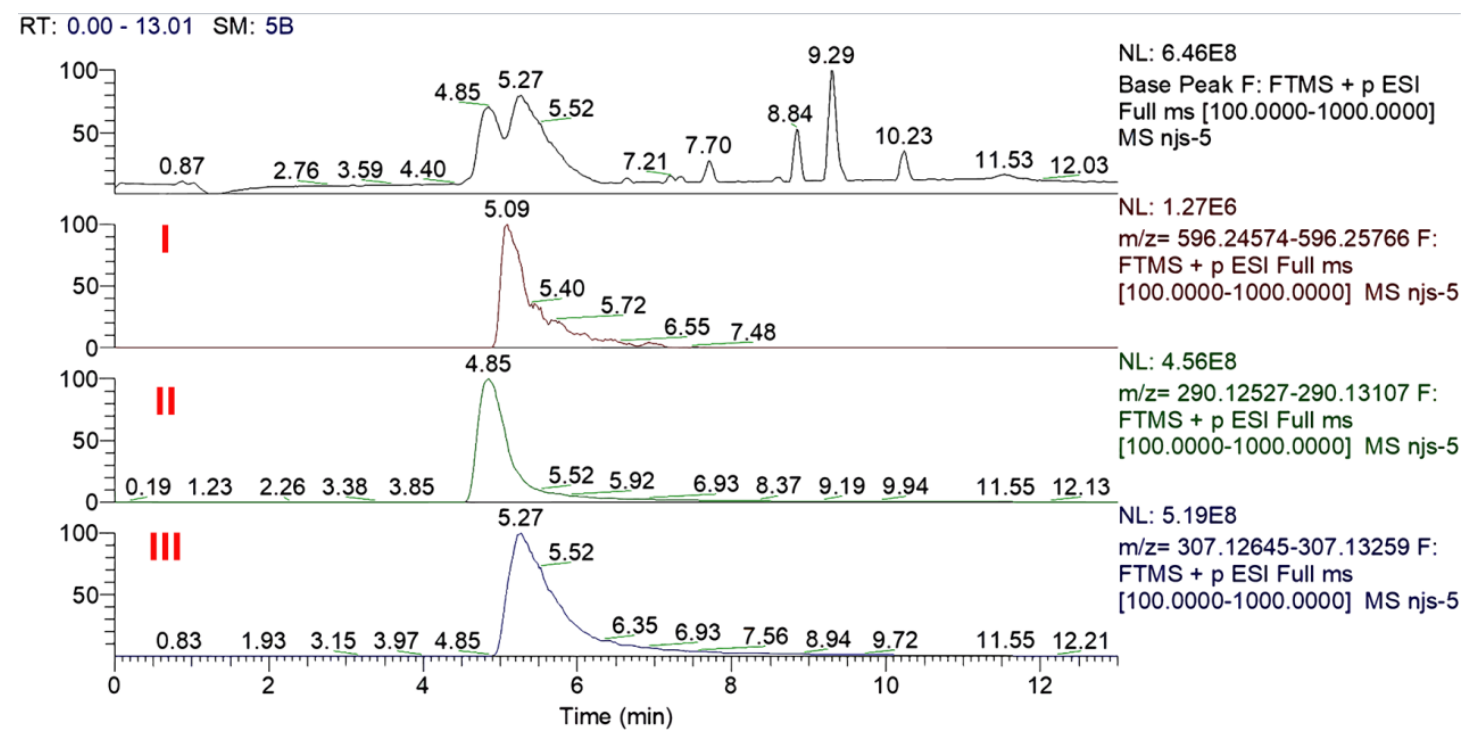

Figure S41a. LC-MS analysis of inactive molecules 4 and 5 in the mixed solution of LB medium and DMF (4:1) in the presence of $\boldsymbol{S}-\boldsymbol{A} \boldsymbol{b}$ to generate active molecule $\mathbf{6}$. 


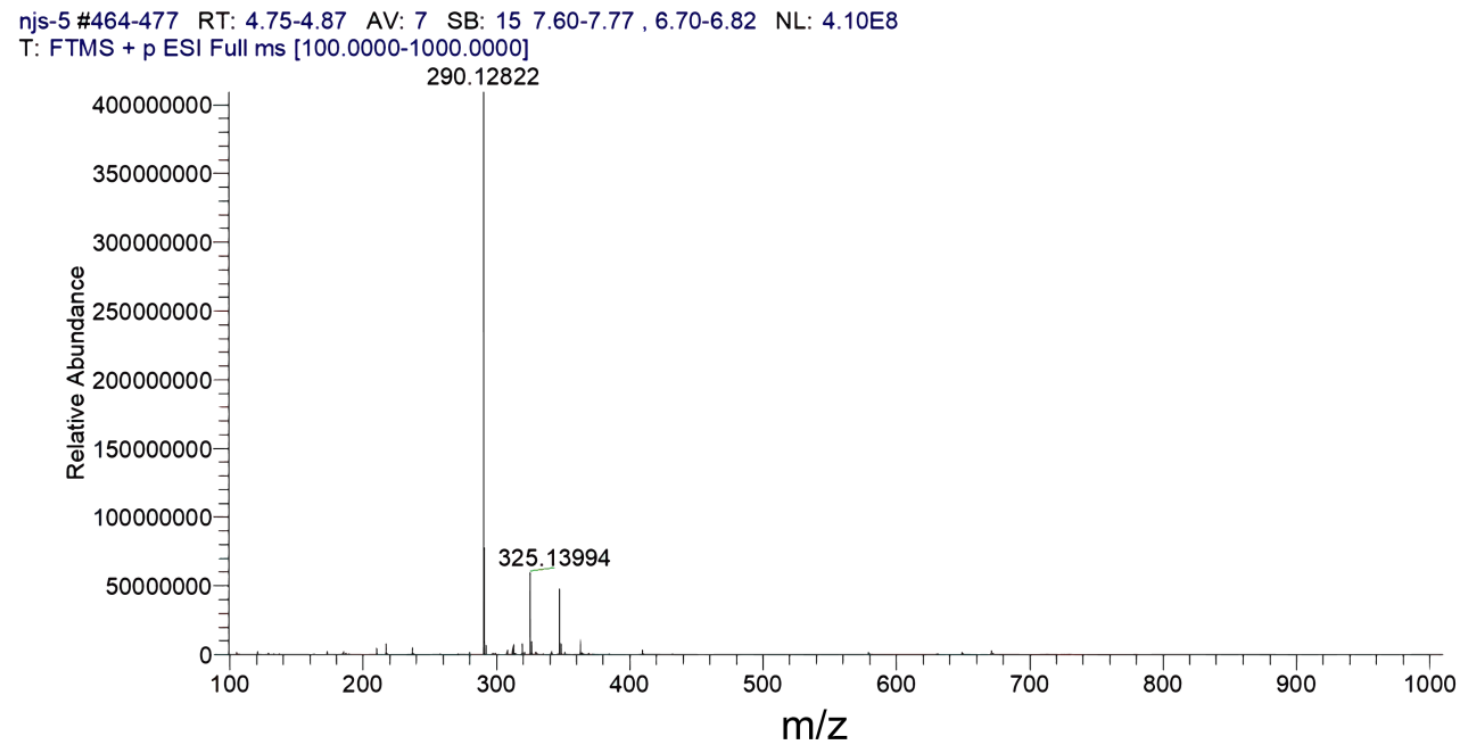

njs-5 \#464-477 RT: 4.75-4.87 AV: 7 SB: 15 7.60-7.77, 6.70-6.82 NL: 4.10E8 T: FTMS + p ESI Full ms [100.0000-1000.0000]

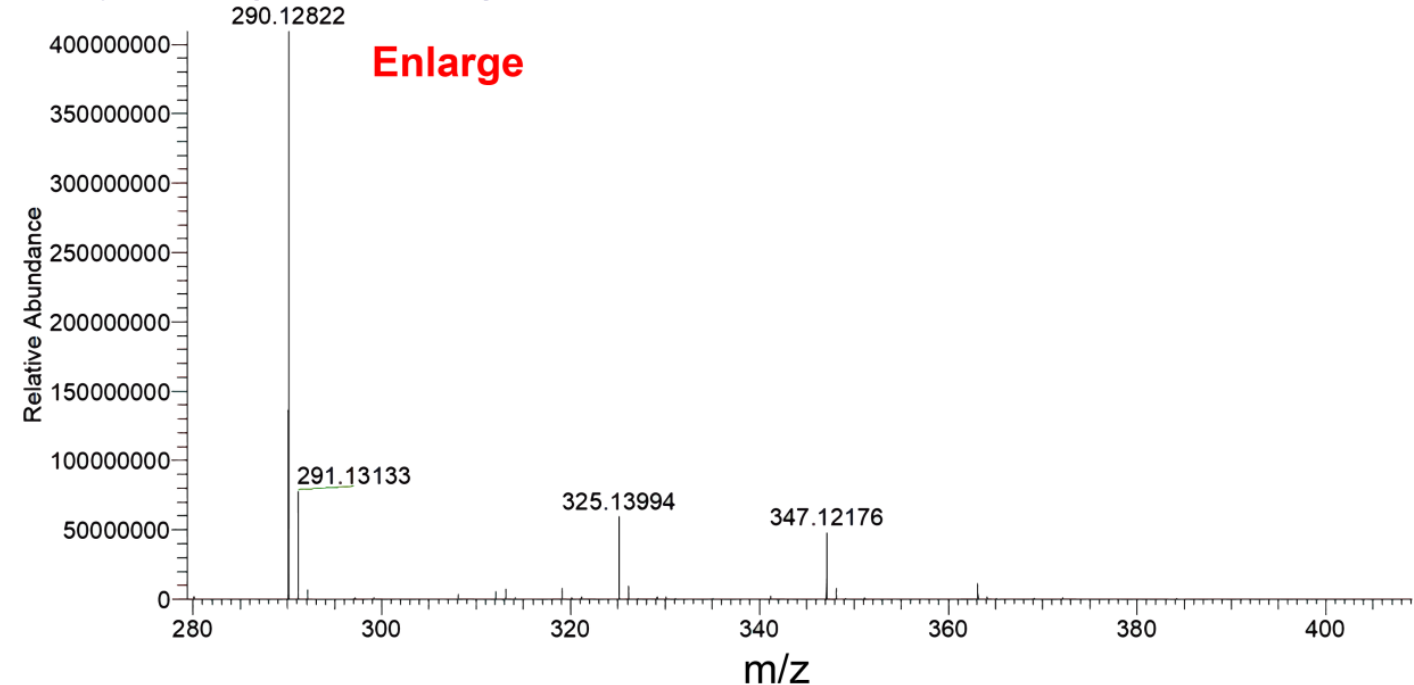

Figure S41b. LC-MS analysis of inactive molecules 4 and 5 in the mixed solution of LB medium and DMF (4:1) in the presence of $\boldsymbol{S}$ - $\boldsymbol{A} \boldsymbol{b}$ to generate active molecule $\mathbf{6}$. The results in this figure indicated the presence of $\mathbf{4}$ in the mixture. 


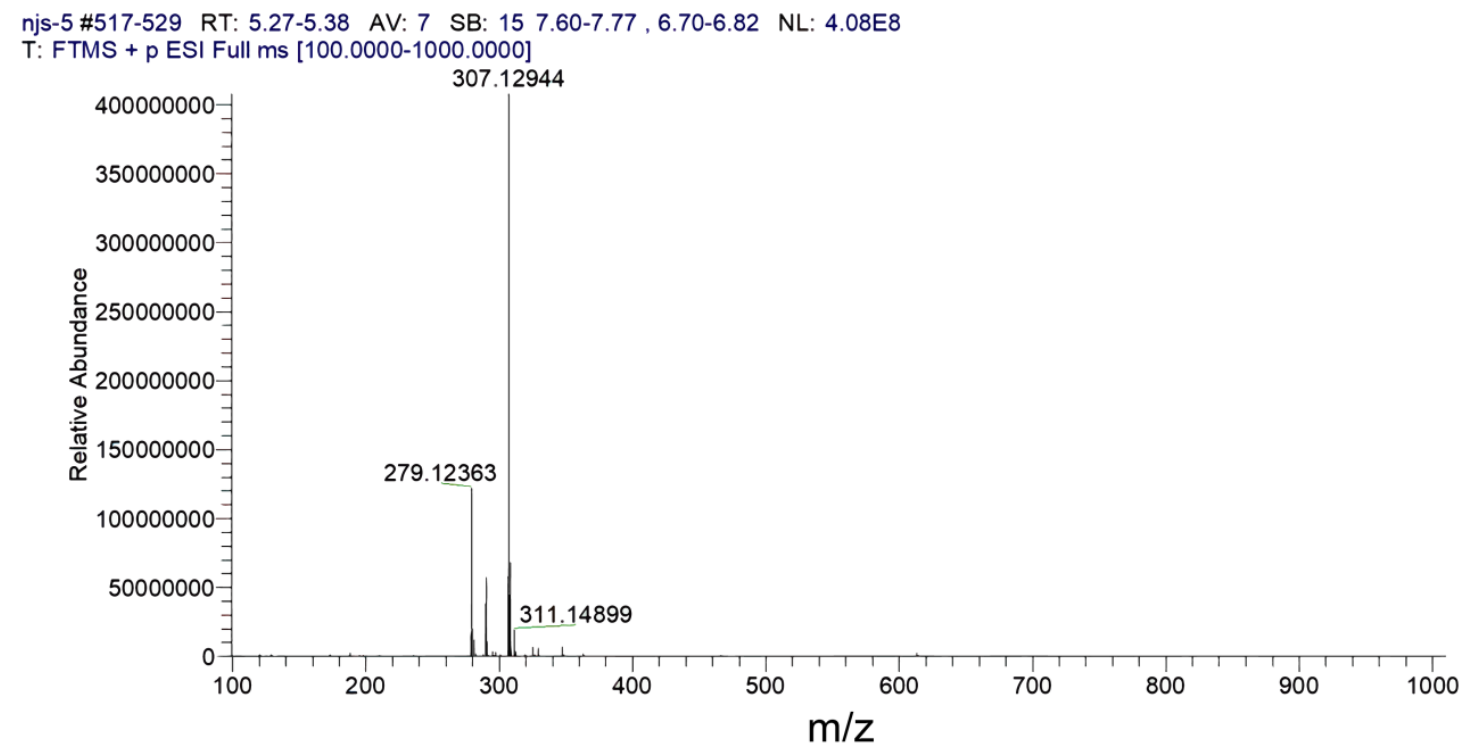

njs-5 \#517-529 RT: $5.27-5.38$ AV: 7 SB: 15 7.60-7.77, 6.70-6.82 NL: 4.08E8 T: FTMS + p ESI Full ms [100.0000-1000.0000]

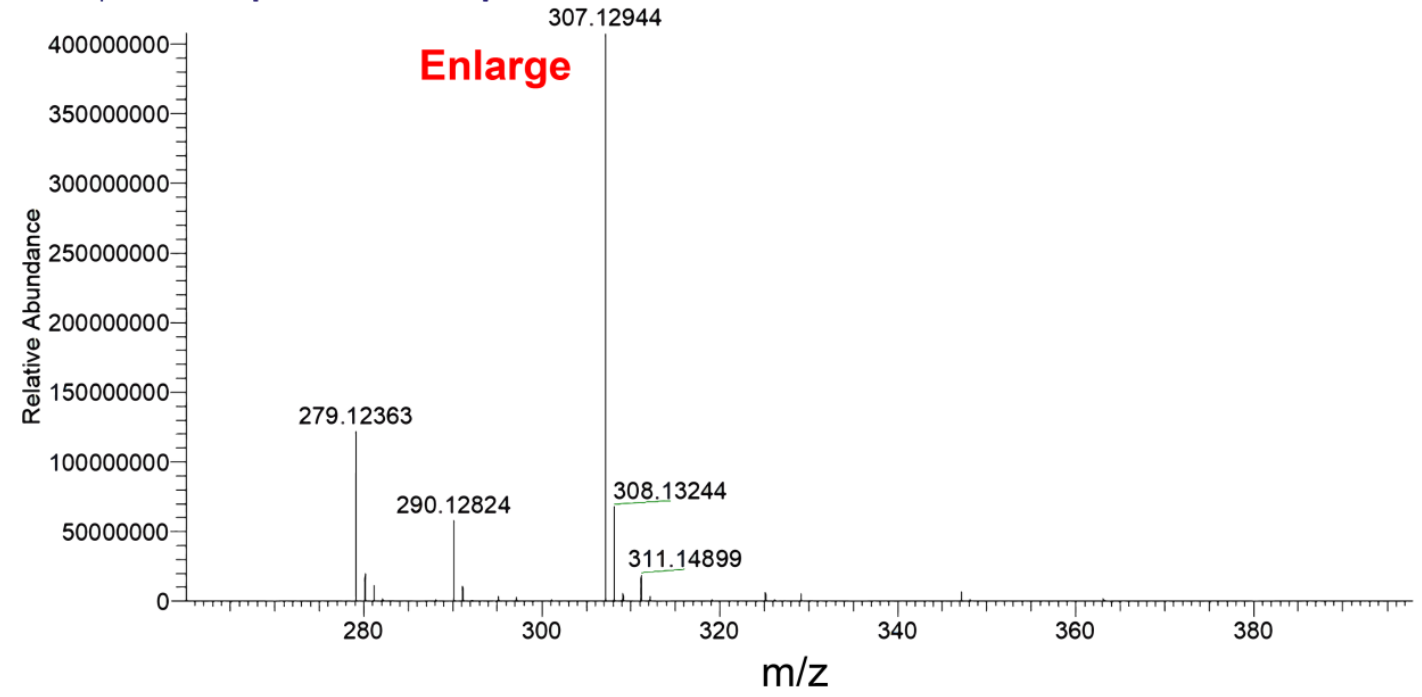

Figure S41c. LC-MS analysis of inactive molecules 4 and 5 in the mixed solution of LB medium and DMF (4:1) in the presence of $\boldsymbol{S}-\boldsymbol{A} \boldsymbol{b}$ to generate active molecule $\mathbf{6}$. The results in this figure indicated the presence of $\mathbf{5}$ in the mixture. 


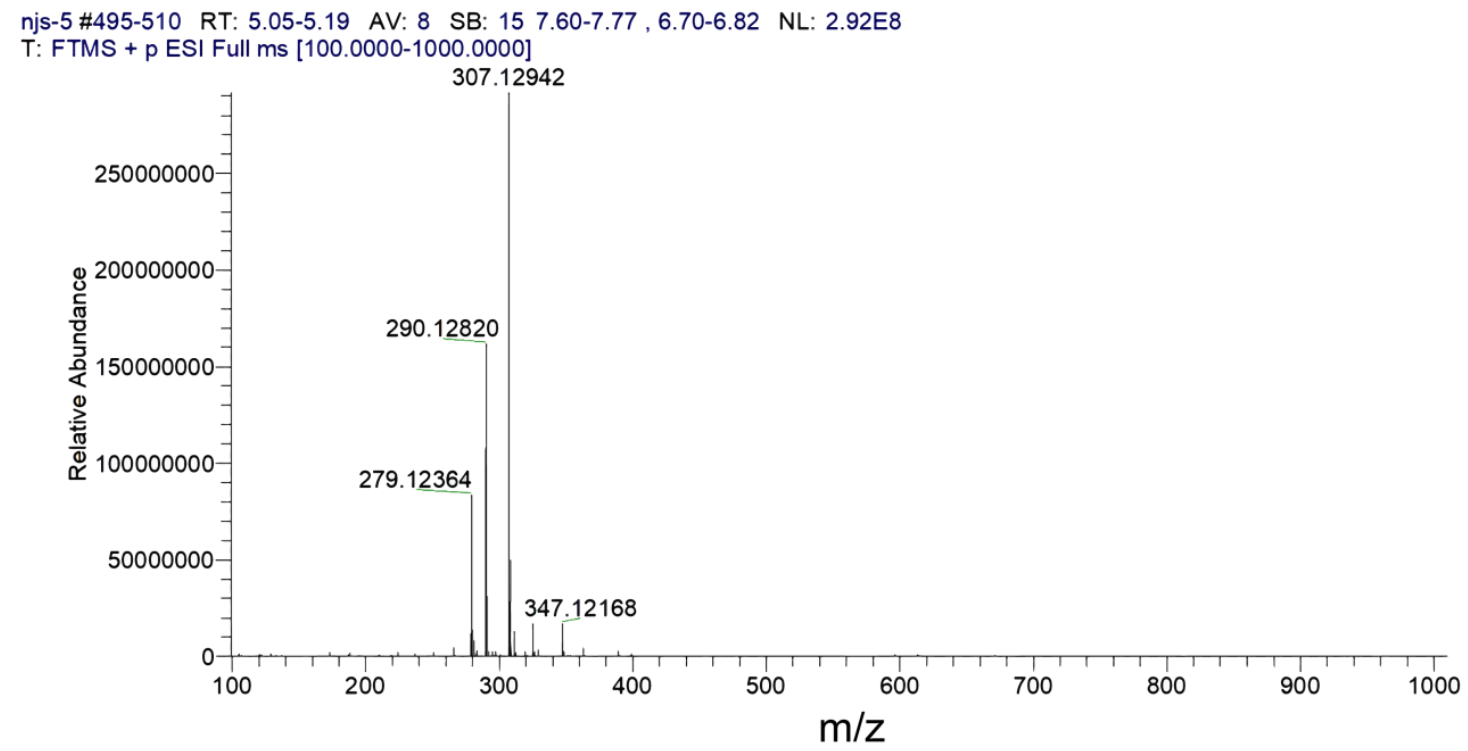

njs-5 \#495-510 RT: $5.05-5.19$ AV: 8 SB: 15 7.60-7.77, 6.70-6.82 NL: 2.92E8 T: FTMS + p ESI Full ms [100.0000-1000.0000]

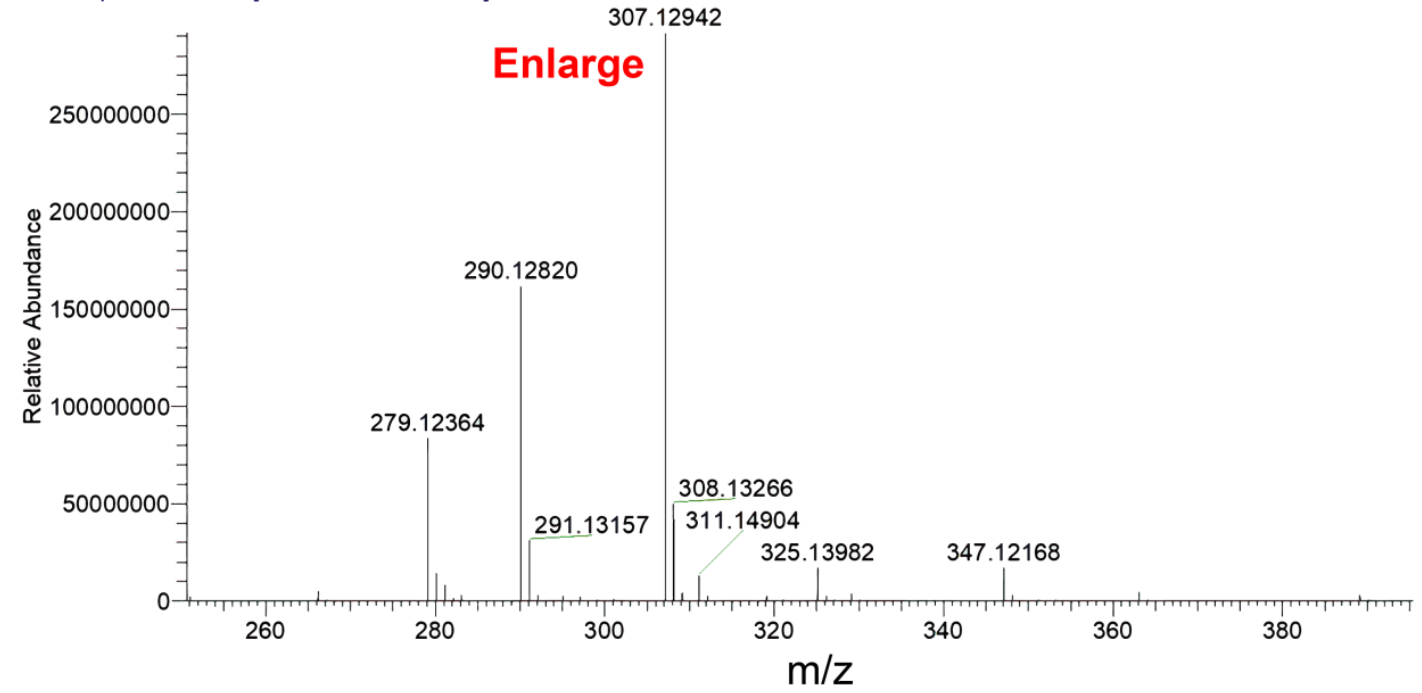

Figure S41d. LC-MS analysis of inactive molecules 4 and $\mathbf{5}$ in the mixed solution of LB medium and DMF (4:1) in the presence of $\boldsymbol{S}-\boldsymbol{A} \boldsymbol{b}$ to generate active molecule $\mathbf{6}$. The results in this figure indicated the presence of $\mathbf{4}$ and $\mathbf{5}$ in the mixture. 


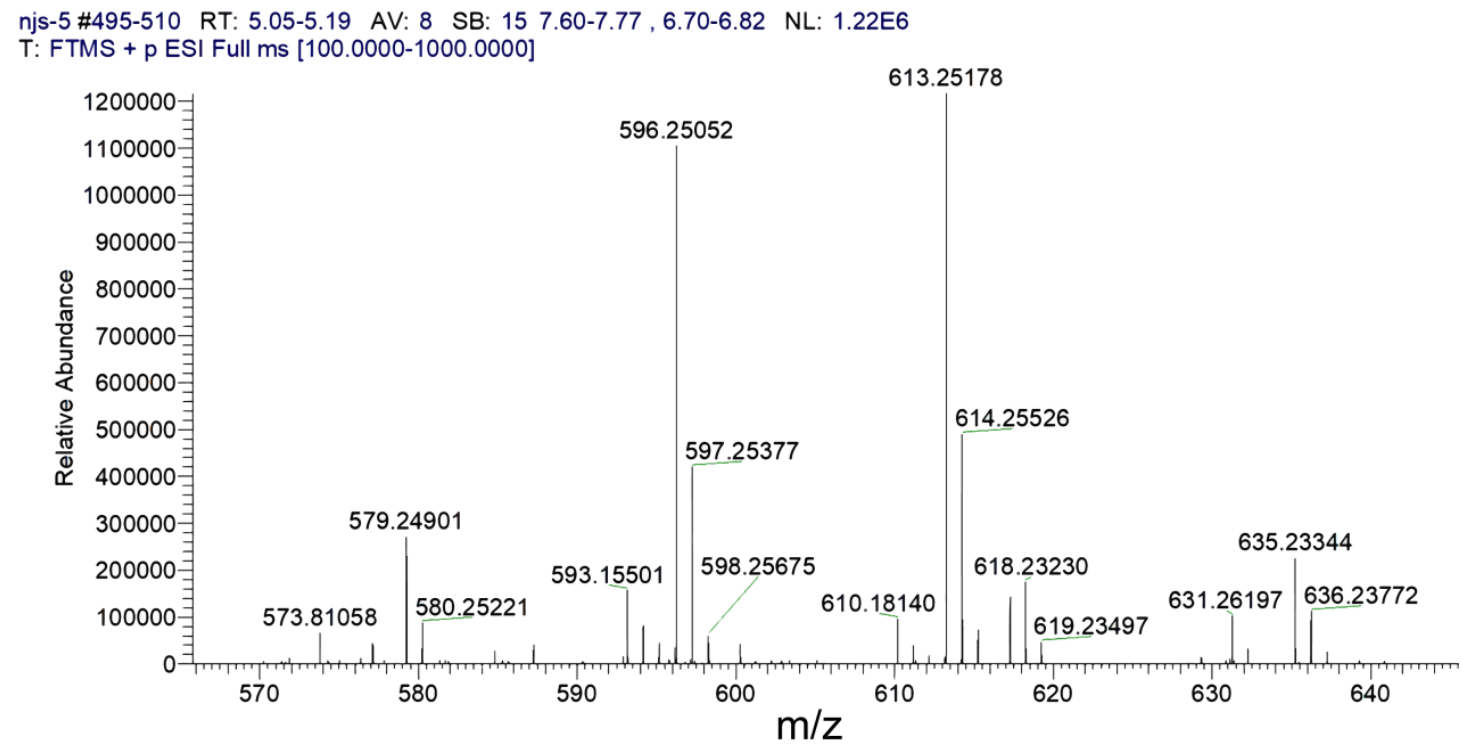

Figure S41e. LC-MS analysis of inactive molecules 4 and $\mathbf{5}$ in the mixed solution of LB medium and DMF (4:1) in the presence of $\boldsymbol{S}$ - $\boldsymbol{A} \boldsymbol{b}$ to generate active molecule $\mathbf{6}$. The final product 6 (596.25052) confirmed that the CuAAC reaction catalyzed by $\boldsymbol{S}$ - $\boldsymbol{A b}$ occurred. 


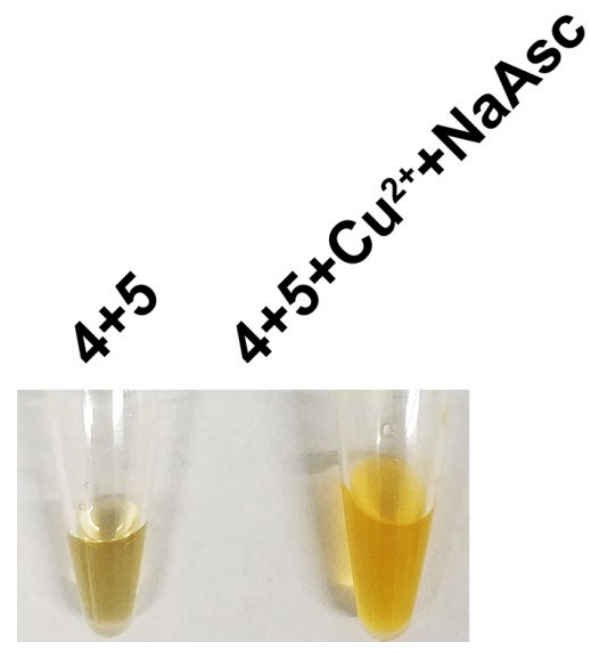

Figure S42. Photographs of precursor molecules $\mathbf{4}$ and $\mathbf{5}$ treated with copper ions and sodium ascorbate. The color change confirmed the formation of product 6.

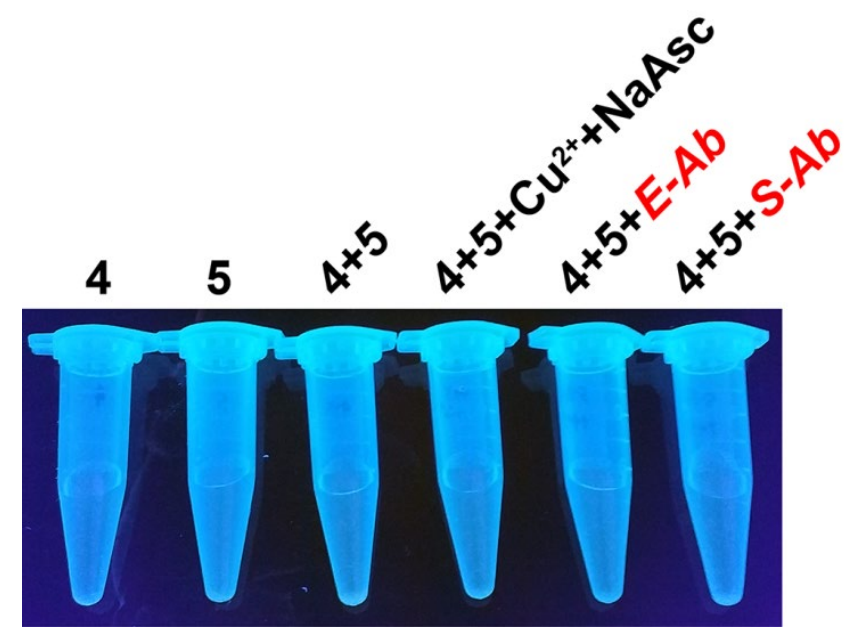

Figure S43. Fluorescence photographs of precursor molecules 4, 5 reacting with different catalysts in $\mathrm{H}_{2} \mathrm{O} / \mathrm{DMSO}(9: 1)$. The results indicated that $\mathbf{4}, \mathbf{5}$ and their reaction products were non-fluorescent. 
a)

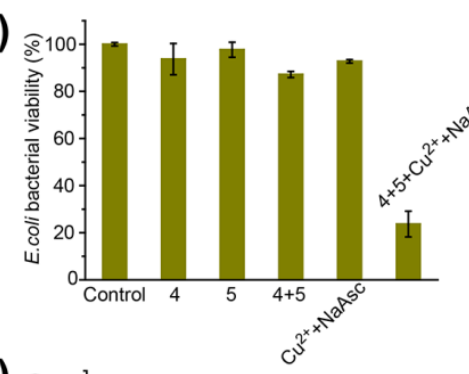

c)

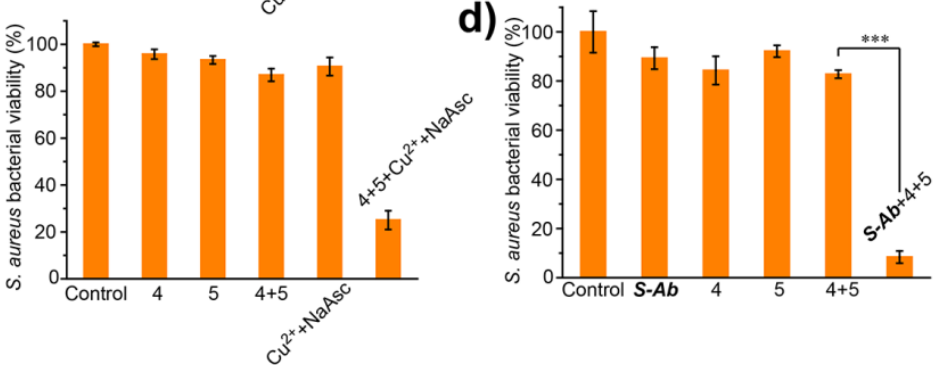

Figure S44. Growth-inhibition assay proved the antibacterial ability of antibody mimics system. In the presence of precursor molecules $\mathbf{4}$ and $\mathbf{5}, \mathrm{Cu}^{2+}+\mathrm{NaAsc}, \boldsymbol{E}-\boldsymbol{A} \boldsymbol{b}$ and $\boldsymbol{S}-\boldsymbol{A} \boldsymbol{b}\left(\mathrm{Cu}^{2+}\right.$, $50 \mu \mathrm{M}, \mathrm{NaAsc}, 100 \mu \mathrm{M} ; \boldsymbol{E}-\boldsymbol{A} \boldsymbol{b}, \boldsymbol{S}-\boldsymbol{A} \boldsymbol{b}, \sim 100 \mu \mathrm{g} / \mathrm{mL} ; \mathbf{4}, \mathbf{5} \sim 70 \mu \mathrm{g} / \mathrm{mL})$ exhibited antibacterial ability. Error bars were taken from three parallel tests per group. 
a)

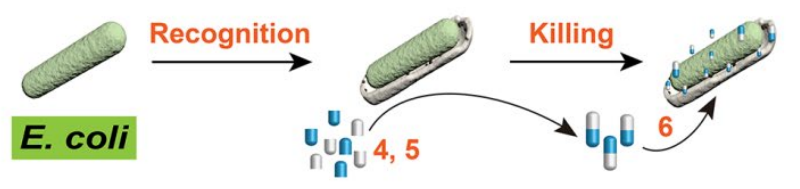

b)

1

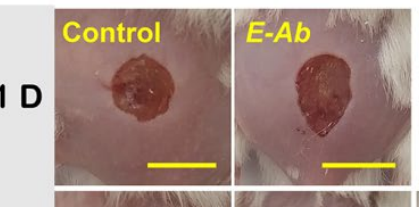

3D

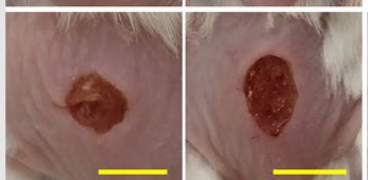

.

$5 \mathrm{D}$

c)

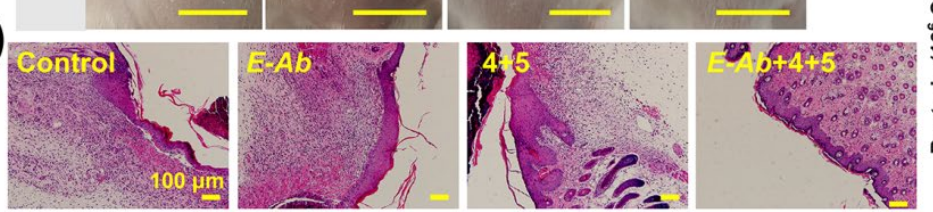

f)

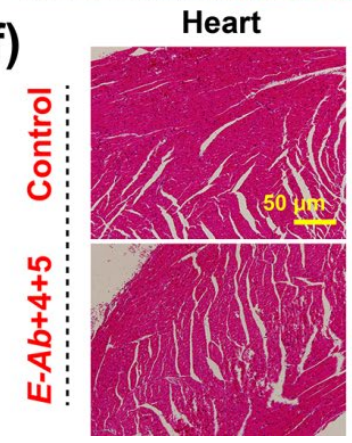

Liver

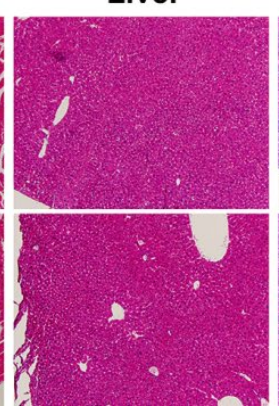

Spleen
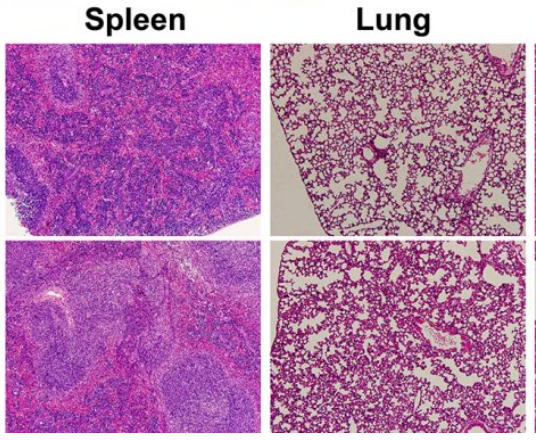

d)

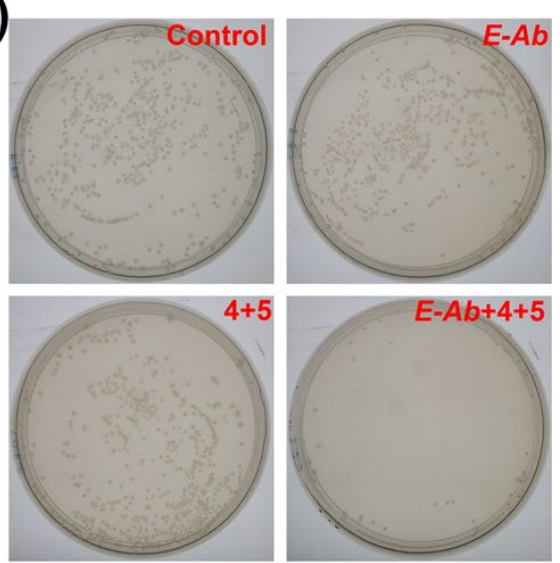

e)
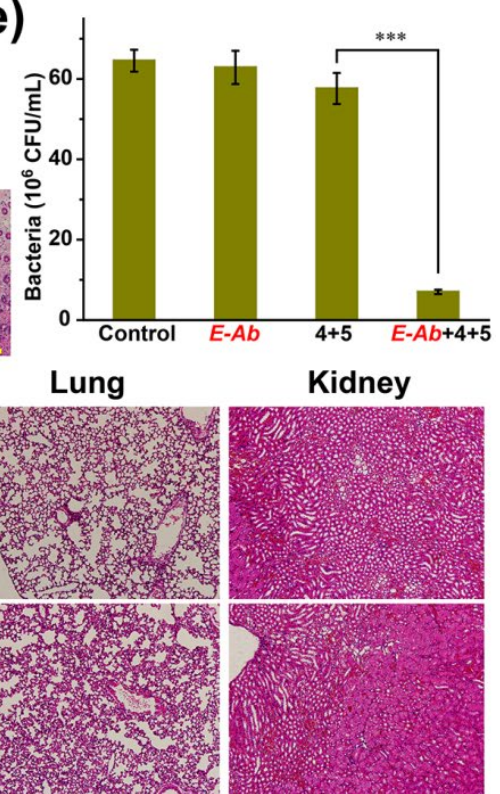

Figure S45. (a) Schematic diagram of $\boldsymbol{E}-\boldsymbol{A} \boldsymbol{b}$ recognized $E$. coli and catalyzed inactive $\mathbf{4}$ and $\mathbf{5}$

to generate active molecule 6. (b) Photographs of wounds from four groups of mice infected

with $E$. coli at 1,3 , and 5 days. The mice were treated with PBS; $\boldsymbol{E}-\boldsymbol{A} \boldsymbol{b} ; \mathbf{4}+\mathbf{5} ; \boldsymbol{E}-\boldsymbol{A} \boldsymbol{b}+\mathbf{4}+\mathbf{5}$, $(\boldsymbol{E}-\boldsymbol{A} \boldsymbol{b}, \sim 100 \mu \mathrm{g} / \mathrm{mL} ; \mathbf{4}, \mathbf{5} \sim 70 \mu \mathrm{g} / \mathrm{mL})$ for 5 days respectively. Scale bars, $0.5 \mathrm{~cm}$. (c) The photomicrographs of H\&E stained skin wound tissue correspond to (b). Scale bars, $100 \mu \mathrm{m}$. (d) Bacteria were isolated from wound tissue after 5 days treatment in each group and cultured on agar plates. (e) The number of surviving bacteria of the wound tissue. Set three mice in each group. ${ }^{* *} p<0.001$. (f) Biosafety evaluation in major organs (heart, liver, spleen, lung, and kidney) after 5 days treatment with PBS, $\boldsymbol{E}-\boldsymbol{A} \boldsymbol{b}+\boldsymbol{4}+\mathbf{5}$. Scale bar, $50 \mu \mathrm{m}$. 

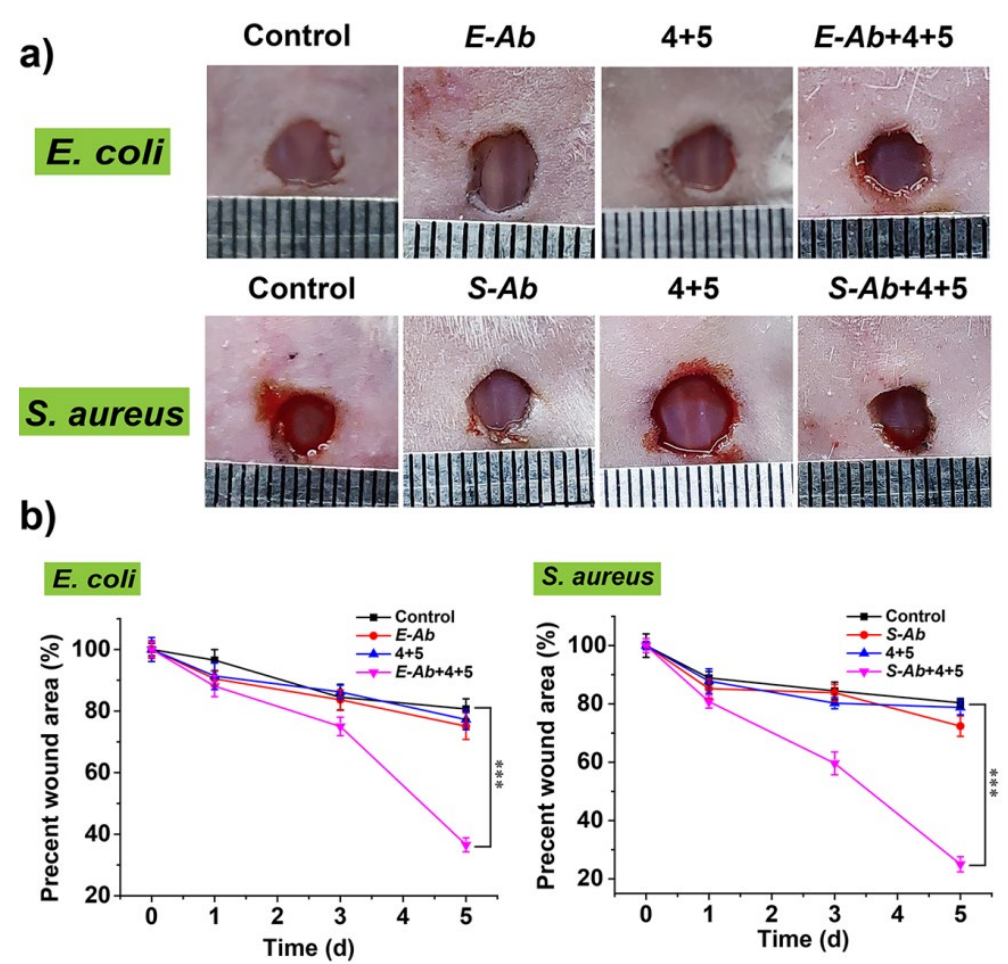

Figure S46. (a) Photographs of wounds from four groups of mice infected with E. coli (Corresponding to Figure S46b) / S. aureus (Corresponding to Figure 4b) on day 0. (b) Percentage wound area of different groups among 5 days (Corresponding to Figure S46b) and Figure $4 \mathrm{~b}$, respectively), ${ }^{* * *} p<0.001$. The results showed that the treatment of $\boldsymbol{E} / \boldsymbol{S}-\boldsymbol{A b}+\mathbf{4}+\mathbf{5}$ group had the best effect in promoting wound healing. 


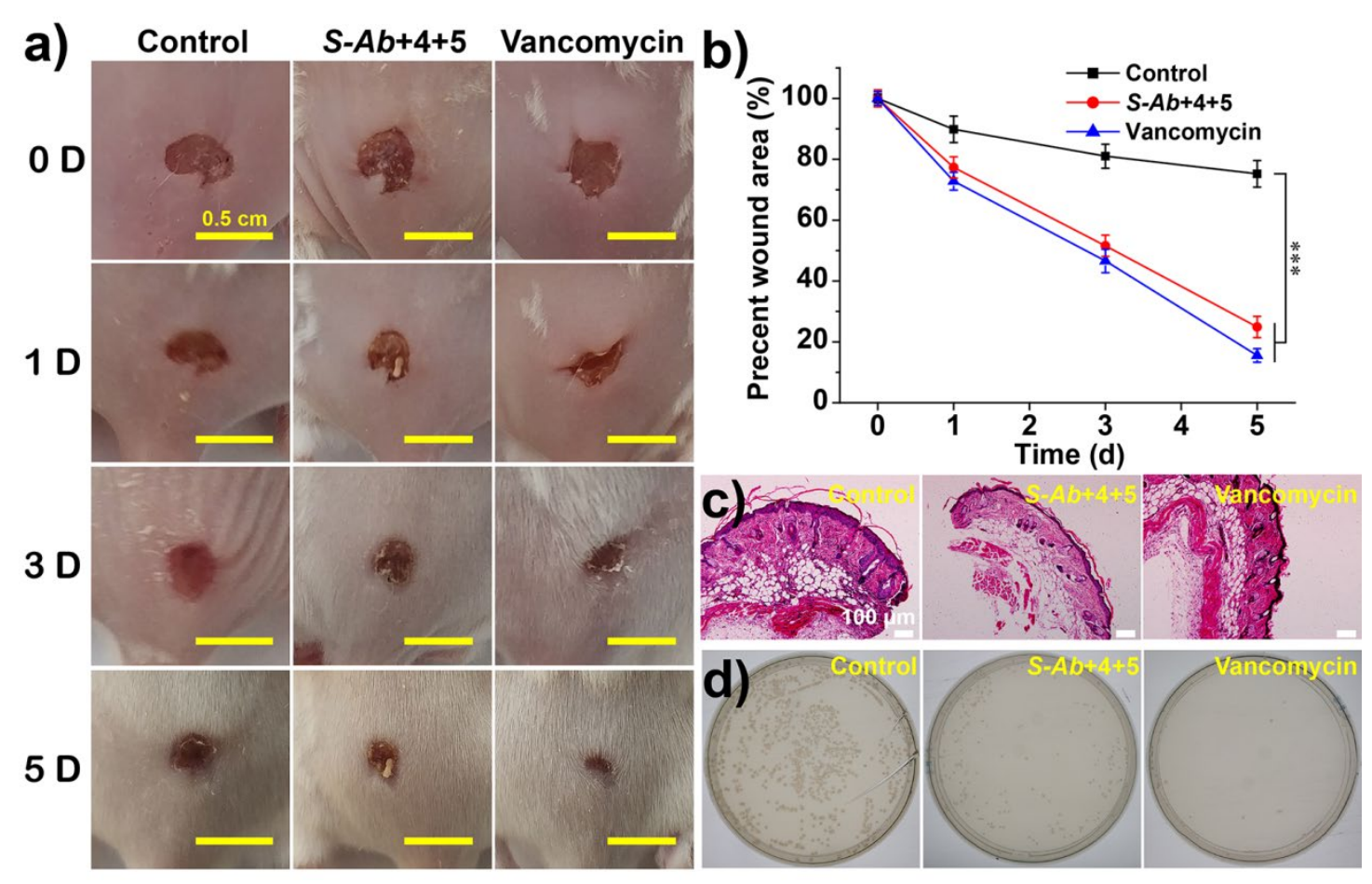

Figure S47. Compared the therapeutic effect of $\boldsymbol{S}-\boldsymbol{A} \boldsymbol{b}+\mathbf{4}+\mathbf{5}$ with vancomycin. (a) Photographs of wounds from three groups of mice infected with $S$. aureus. The mice were treated with PBS; $\boldsymbol{S}-\boldsymbol{A} \boldsymbol{b}+\mathbf{4}+\mathbf{5}(\boldsymbol{S}-\boldsymbol{A} \boldsymbol{b}, \sim 100 \mu \mathrm{g} / \mathrm{mL} ; \mathbf{4}, \mathbf{5} \sim 70 \mu \mathrm{g} / \mathrm{mL})$, Vancomycin $(\sim 16 \mu \mathrm{g} / \mathrm{mL} ;) \quad$ (b) Percentage wound area of different groups among 5 days. $* * * p<0.001$. (c) The photomicrographs of H\&E stained skin wound tissue correspond to (a). Scale bars, $100 \mu \mathrm{m}$. (d) Bacteria were isolated from wound tissue and cultured on agar plates. 


\section{References}

(1) Bai, Y.; Feng, X.; Xing, H.; Xu, Y.; Kim, B. K.; Baig, N.; Zhou, T.; Gewirth, A. A.; Lu, Y.; Oldfield, E.; Zimmerman, S. C. A Highly Efficient Single-Chain Metal-Organic Nanoparticle Catalyst for Alkyne-Azide "Click" Reactions in Water and in Cells. J. Am. Chem. Soc. 2016, 138, 11077-11080.

(2) Zhu, W.; Wang, Y.; Li, K.; Gao, J.; Huang, C. H.; Chen, C. C.; Ko, T. P.; Zhang, Y.; Guo, R. T.; Oldfield, E. Antibacterial Drug Leads: DNA and Enzyme Multitargeting. J. Med. Chem. 2015, 58, 1215-27.

(3) Panchal, R. G.; Ulrich, R. L.; Lane, D.; Butler, M. M.; Houseweart, C.; Opperman, T.; Williams, J. D.; Peet, N. P.; Moir, D. T.; Nguyen, T.; Gussio, R.; Bowlin, T.; Bavari, S. Novel Broad-Spectrum Bis-(Imidazolinylindole) Derivatives with Potent Antibacterial Activities against Antibiotic-Resistant Strains. Antimicrob. Agents Chemother. 2009, 53, 4283-91. 